\title{
NUMERICAL SIMULATION OF LIQUID FUEL PRECHAMBER IGNITION
}

\author{
By \\ Levon Larson, B. Eng \\ Aerospace Engineering \\ Ryerson University, 2011
}

A thesis presented to Ryerson University

in partial fulfillment of the

requirements for the degree of

Master of Applied Science

in the Program of

Aerospace Engineering

Toronto, Ontario, Canada

(C) Levon Larson 2013 
I hereby declare that I am the sole author of this thesis. This is a true copy of the thesis, including any required final revisions, as accepted by my examiners.

I authorize Ryerson University to lend this thesis to other institutions or individuals for the purpose of scholarly research.

I further authorize Ryerson University to reproduce this thesis by photocopying or by other means, in total or in part, at the request of other institutions or individuals for the purpose of scholarly research.

I understand that my thesis may be made electronically available to the public. 


\title{
Abstract
}

\section{NUMERICAL SIMULATION OF LIQUID FUEL PRECHAMBER IGNITION}

\author{
Master of Applied Science \\ 2013 \\ Levon Larson \\ Aerospace Engineering \\ Ryerson University
}

A Computational Fluid Dynamics (CFD) model was built that simulates the transient, compressible, reacting, multi-phase environment that exists within a reciprocating engine's combustion chamber(s). ANSYS Fluent v13.0 was used with the Euler-Lagrangian Discrete Phase Model (DPM), the Shell autoignition model, and the Large Eddy Simulation (LES) method of turbulence modeling. Validation of the spray dynamics was performed by comparing simulation results with experiments of liquid and vapour penetration length of an $n$-Heptane spray experiment done by Sandia National Laboratories. It was found that LES produced more accurate results than several Reynolds Averaged Navier-Stokes (RANS) models. The Shell autoignition model was coded to function with $\mathrm{C}_{10.17} \mathrm{H}_{19.91}$ and compared with experimental ignition results in a Rapid Compression Machine (RCM) environment. All of the above models were then combined to simulate a directly-fueled lean-burn combustion prechamber configuration wherein the effects of spray angle, timing, and duration were studied. 


\section{Acknowledgements}

The author would like to thank: Dr. Seth Dworkin and Dr. Paul Walsh for their expertise and continued guidance for the duration of this project and Mitacs for the experience offered through their innovative research internship program. 


\section{Table of Contents}

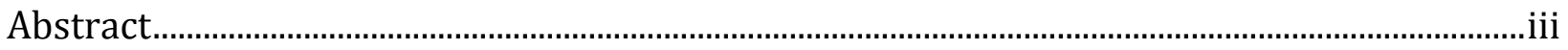

Acknowledgements .........................................................................................................................iv

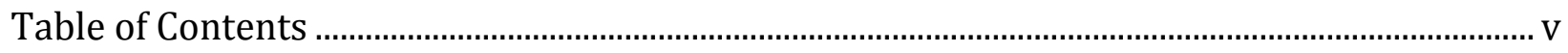

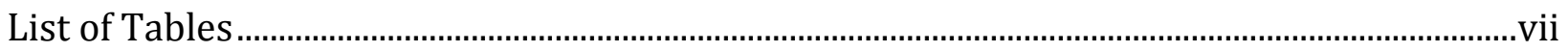

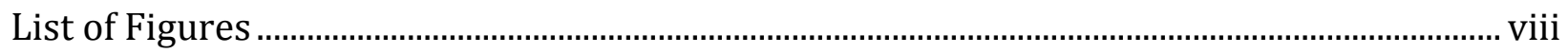

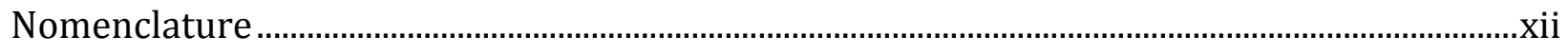

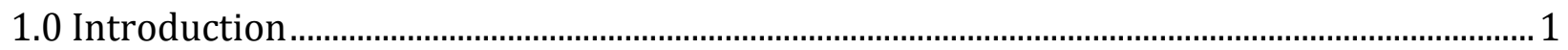

2.0 Literature Review .........................................................................................................

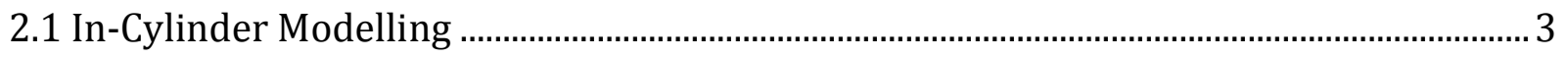

2.2 Prechamber Review ............................................................................................................... 8

2.3 Previous Prechamber CFD Modelling ………………………................................................. 14

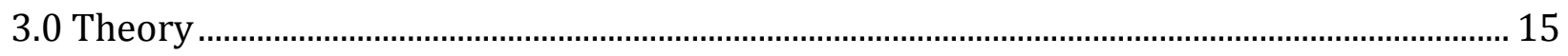

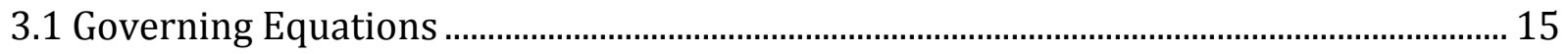

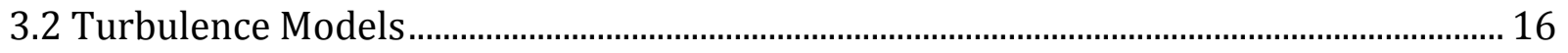

3.2.1 Reynolds Averaged Navier-Stokes .............................................................................. 17

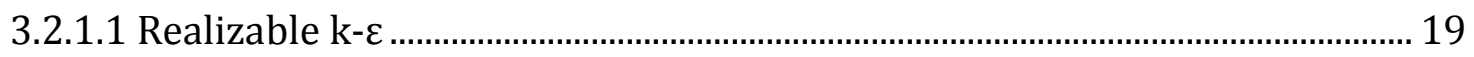

3.2.2 Large Eddy Simulation............................................................................................ 21

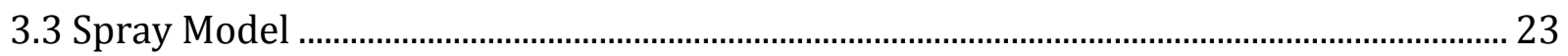

3.3.1 Particle Motion .................................................................................................................. 25

3.3.2 Particle Drag Laws ...................................................................................................... 26

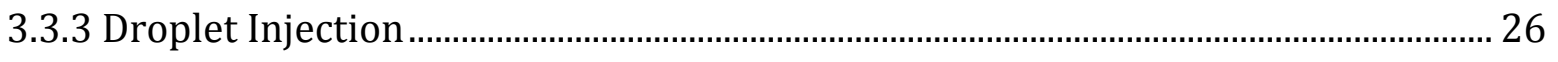

3.3.4 Breakup Models ..................................................................................................... 28

3.3.4.1 Kelvin-Helmholtz Breakup Model ........................................................................ 30 
3.3.4.2 Rayleigh-Taylor Breakup Model........................................................................... 32

3.4 Eddy Dissipation Concept.................................................................................................... 33

3.4.1 Shell Autoignition Model ................................................................................................ 34

3.4.2 High Temperature Model .............................................................................................. 39

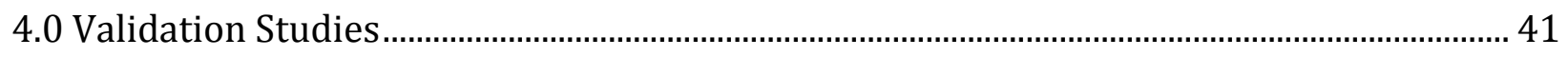

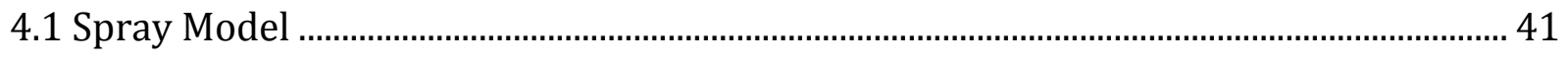

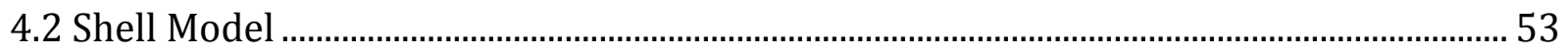

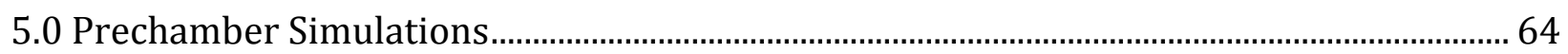

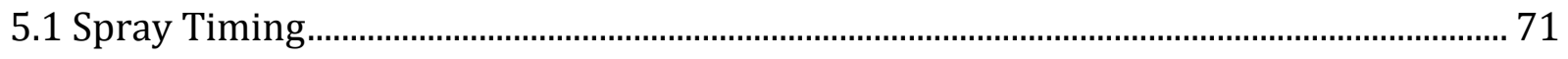

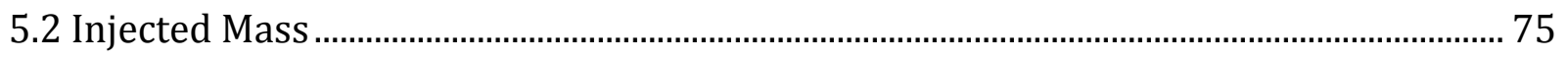

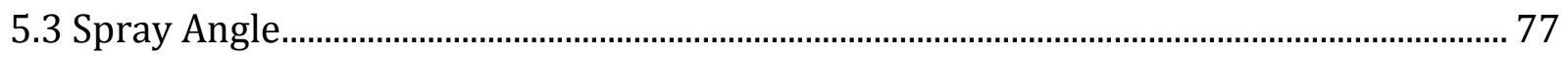

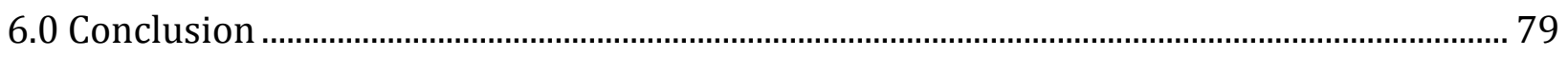

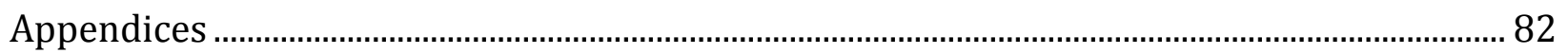

Appendix A - Shell Model Chemistry Source Terms ………………………………………....... 82

Appendix B - First and Second Stage Delay Simulations Results............................................ 83

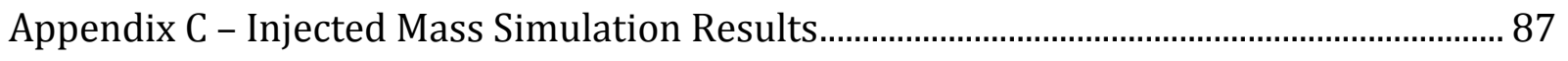

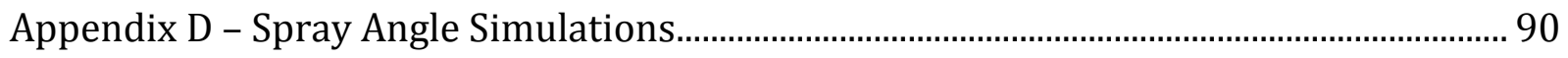

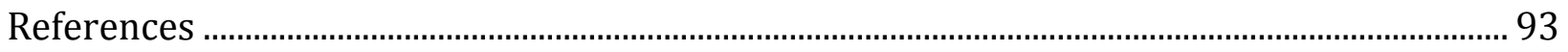




\section{List of Tables}

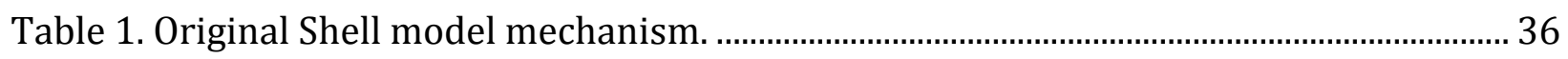

Table 2. Schäpertöns/Lee mechanism............................................................................................ 37

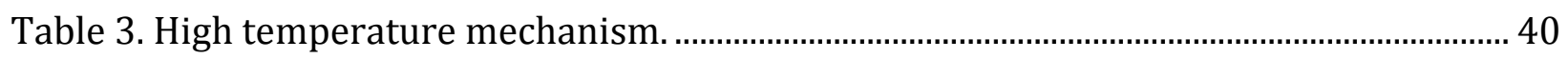

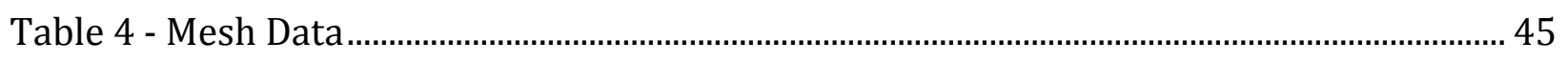

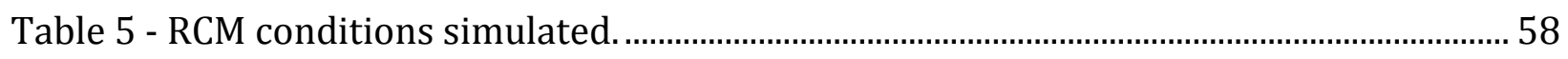

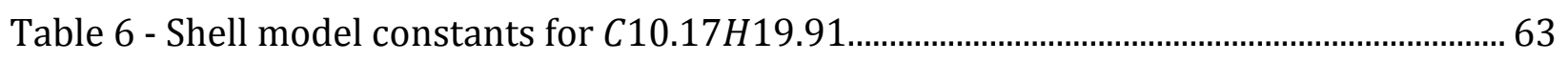

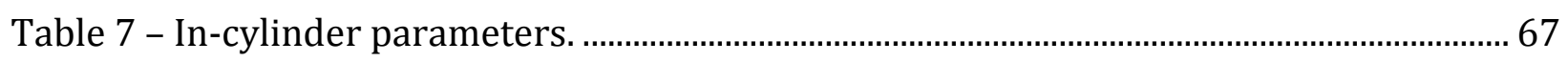

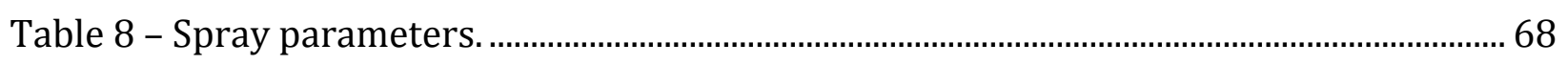




\section{List of Figures}

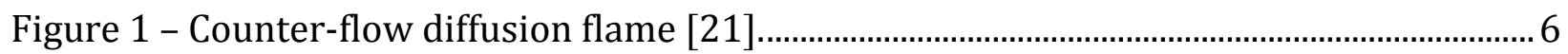

Figure 2 - Cylinder head and prechamber from Ricardo Dolphin engine [32] ........................ 10

Figure 3 - Cylinder head and prechamber from Honda CVCC [33]........................................... 11

Figure 4 - Cylinder head and turbulence generating pot from Toyota [34]............................. 11

Figure 5 - Cylinder head and prechamber from GM [37] .......................................................... 12

Figure 6 - Cylinder head and auxiliary chamber from Porsche [38] ........................................... 12

Figure 7 - Cylinder and prechamber demonstrating the LAG process [39]............................ 13

Figure 8 - Visual representation of the mean and fluctuating component of turbulent

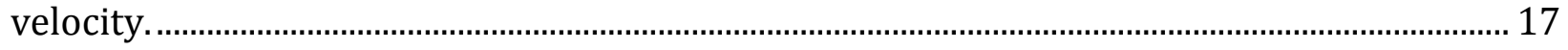

Figure 9 - Blob method approximation of the liquid core [21] ..................................................2

Figure 10 - CFD illustration of injected droplet size [62].......................................................... 27

Figure 11 - Illustration of breakup model regions [62] .............................................................. 29

Figure 12 - Characteristic two-stage ignition event [70]........................................................... 35

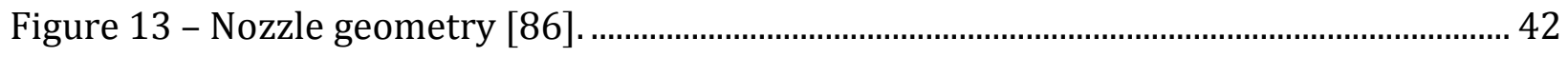

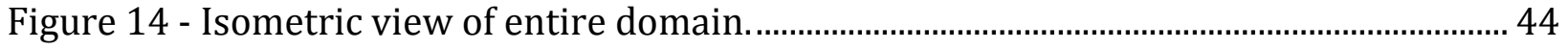

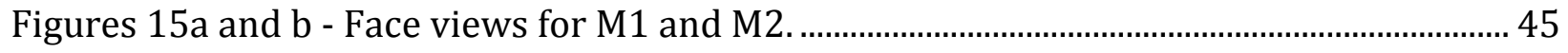

Figure 16 - Vapour length validation results for the LES model. ............................................... 47

Figure 17 - Liquid length validation results for the LES model. ................................................ 48

Figure 18 - Vapour length validation results for the Realizable k- $\varepsilon$ model............................... 48

Figure 19 - Liquid length validation results for the Realizable k- $\varepsilon$ model................................. 49

Figure 20 - Vapour length validation results for the Reynolds Stress Model........................... 49 
Figure 21 - Liquid length validation results for the Reynolds Stress Model............................. 50

Figure 22 - Vapour length comparison with all turbulence models........................................... 50

Figure 23 - Liquid length comparison with all turbulence models. ............................................ 51

Figure 24 - Vapour and liquid length validation for final LES case. ........................................... 52

Figure 25 - RCM Schematic [87]......................................................................................... 54

Figure 26 - Isometric view of RCM mesh. …………….......................................................... 56

Figure 27 - Shell model comparison with RCM experiment......................................................... 59

Figure 28 - Configuration 1 total ignition delay, $\Phi=1.12, P c=7$ bar ................................... 59

Figure 29 - Configuration 2 total ignition delay, $\Phi=1.12, P c=15$ bar................................. 60

Figure 30 - Configuration 3 total ignition delay, $\Phi=0.76, P c=7$ bar..................................... 60

Figure 31 - Configuration 4 total ignition delay, $\Phi=0.76, P c=15$ bar................................ 61

Figure 32 - Position of autoignition prechamber in cylinder head [93].................................... 64

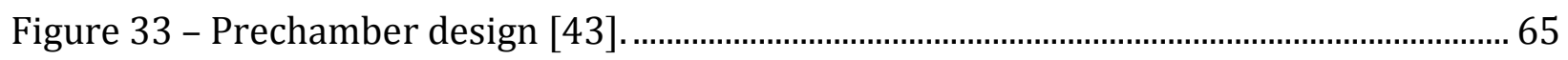

Figure 34- Prechamber mesh where purple section is heated to promote ignition................ 66

Figure 35- Equivalence ratios for all spray configurations simulated. ....................................... 70

Figure 36- Pressures for all spray configurations simulated...................................................... 70

Figure 37- Pressures and equivalence ratios for all sprays lasting $9^{\circ} \mathrm{CA}$ with a $10^{\circ}$ cone. . 71

Figure 38 - Pressures and equivalence ratios for all sprays lasting $18^{\circ} \mathrm{CA}$ with a $10^{\circ}$ cone. 72

Figure 39- Pressures and equivalence ratios for all sprays lasting $9^{\circ} \mathrm{CA}$ with a $30^{\circ}$ cone. . 73

Figure 40 - Pressures and equivalence ratios for all sprays lasting $18^{\circ} \mathrm{CA}$ with a $30^{\circ}$ cone. 74

Figure 41- Pre-ignition temperatures for all sprays. ………………………………………....... 75

Figure 42 - Pressures and equivalence ratios for sprays beginning at $-90^{\circ} \mathrm{CA}$ with a cone

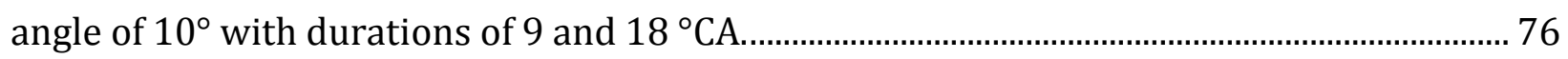


Figure 43- Pressures and equivalence ratios for sprays beginning at $-90^{\circ} \mathrm{CA}$ with a

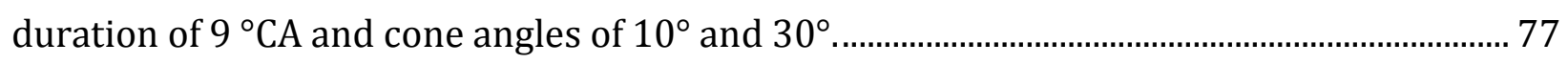

Figure B.1 - Configuration 1 first stage ignition delay ................................................................ 83

Figure B.2 - Configuration 1 second stage ignition delay. ........................................................... 83

Figure B.3 - Configuration 2 first stage ignition delay................................................................ 84

Figure B.4 - Configuration 2 second stage ignition delay. ............................................................ 84

Figure B.5 - Configuration 3 first stage ignition delay.............................................................. 85

Figure B.6 - Configuration 3 second stage ignition delay. ......................................................... 85

Figure B.7 - Configuration 4 first stage ignition delay................................................................ 86

Figure B.8 - Configuration 4 second stage ignition delay. .......................................................... 86

Figure C.1- Pressures and equivalence ratios for sprays beginning at $-90^{\circ} \mathrm{CA}$ with a cone

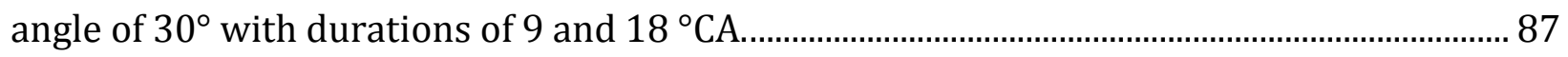

Figure C.2- Pressures and equivalence ratios for sprays beginning at $-72^{\circ} \mathrm{CA}$ with a cone

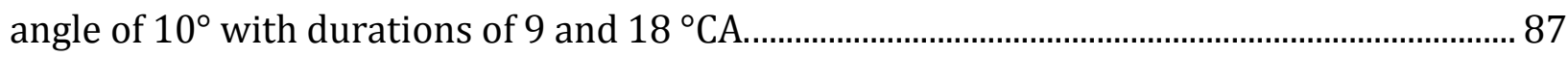

Figure C.3- Pressures and equivalence ratios for sprays beginning at $-72^{\circ} \mathrm{CA}$ with a cone

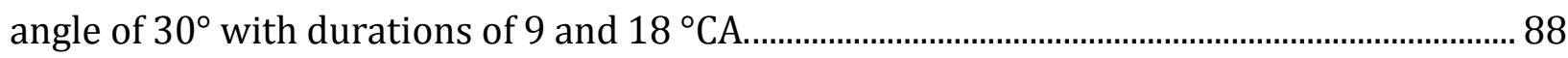

Figure C.4- Pressures and equivalence ratios for sprays beginning at $-45^{\circ} \mathrm{CA}$ with a cone

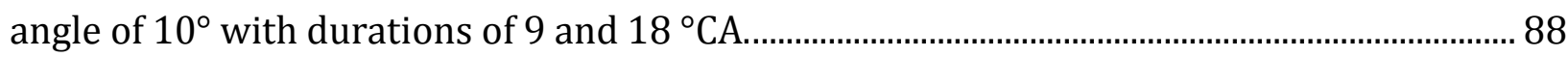

Figure C.5- Pressures and equivalence ratios for sprays beginning at $-45^{\circ} \mathrm{CA}$ with a cone

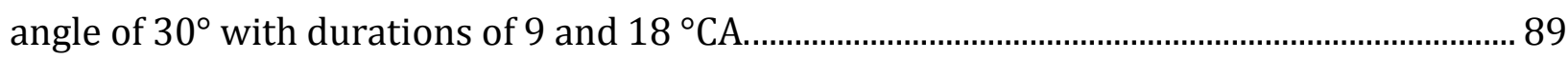

Figure D.1 - Pressures and equivalence ratios for sprays beginning at $-90^{\circ} \mathrm{CA}$ with a duration of $18^{\circ} \mathrm{CA}$ and cone angles of $10^{\circ}$ and $30^{\circ}$. 
Figure D. 2 - Pressures and equivalence ratios for sprays beginning at $-72^{\circ} \mathrm{CA}$ with a

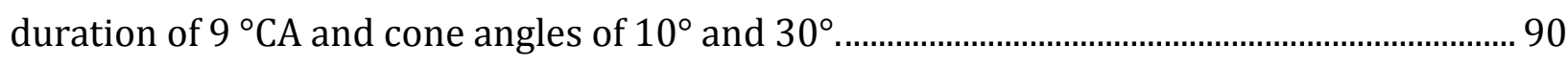

Figure D. 3 - Pressures and equivalence ratios for sprays beginning at $-72^{\circ} \mathrm{CA}$ with a

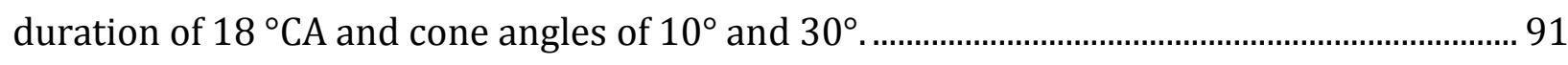

Figure D. 4 - Pressures and equivalence ratios for sprays beginning at $-72^{\circ} \mathrm{CA}$ with a

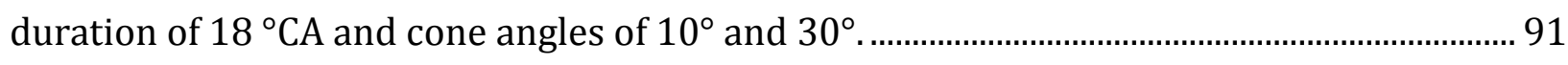

Figure D.5 - Pressures and equivalence ratios for sprays beginning at $-72^{\circ} \mathrm{CA}$ with a

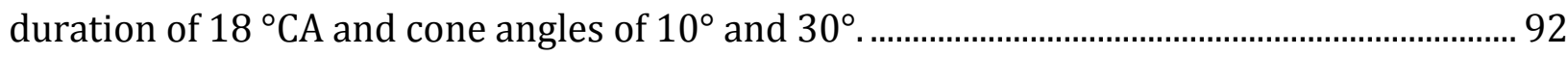




\section{Nomenclature}

\begin{tabular}{|c|c|c|}
\hline Variable & Section & Description \\
\hline$\rho$ & 3.1 & Fluid density \\
\hline$u_{i}$ & 3.1 & Fluid velocity \\
\hline$\delta_{i j}$ & 3.1 & Kronecker delta \\
\hline$p$ & 3.1 & Fluid pressure \\
\hline$\mu$ & 3.1 & Dynamic viscosity \\
\hline$\lambda$ & 3.1 & Second viscosity \\
\hline$g_{j}$ & 3.1 & Gravity force \\
\hline$e$ & 3.1 & Internal energy \\
\hline$q$ & 3.1 & Conductive heat flux \\
\hline$Y_{k}$ & 3.1 & Mass fraction of species k \\
\hline$V_{k, i}$ & 3.1 & Diffusive velocity of species $\mathrm{k}$ \\
\hline$\dot{\omega}_{k}$ & 3.1 & Source/Sink of species k \\
\hline$\mu_{t}$ & 3.2 .1 & Turbulent viscosity \\
\hline$k$ & 3.2 .1 .1 & Turbulent kinetic energy \\
\hline$\varepsilon$ & 3.2 .1 .1 & Turbulent dissipation rate \\
\hline$G_{k}$ & 3.2.1.1 & $\begin{array}{l}\text { Production of } k \text { from mean } \\
\text { velocity gradients }\end{array}$ \\
\hline$G_{b}$ & 3.2.1.1 & Production of $k$ from buoyancy \\
\hline$Y_{M}$ & 3.2 .1 .1 & Fluctuating dilatation \\
\hline$\sigma_{k}$ & 3.2.1.1 & Turbulent Prandtl Number for $k$ \\
\hline$\sigma_{\varepsilon}$ & 3.2.1.1 & Turbulent Prandtl Number for $\varepsilon$ \\
\hline$S_{k}$ & 3.2 .1 .1 & Source term for $k$ \\
\hline
\end{tabular}




\begin{tabular}{|c|c|c|}
\hline$S_{\varepsilon}$ & 3.2.1.1 & Source term for $\varepsilon$ \\
\hline$C_{2}$ & 3.2.1.1 & Model constant for $k-\varepsilon$ \\
\hline$C_{3 \varepsilon}$ & 3.2.1.1 & Model constant for $k-\varepsilon$ \\
\hline$A_{o}$ & 3.2.1.1 & Model constant for $k-\varepsilon$ \\
\hline$\overline{\overline{\Omega_{l \jmath}}}$ & 3.2.1.1 & Mean rate of rotation tensor \\
\hline$\omega_{k}$ & 3.2.1.1 & Angular velocity \\
\hline$G\left(x, x^{\prime}\right)$ & 3.2 .2 & LES filter function \\
\hline$\tau_{i j}$ & 3.2 .2 & Subgrid Stress \\
\hline$\mu_{s g s}$ & 3.2 .2 & Subgrid turbulent viscosity \\
\hline$\overline{S_{i j}}$ & 3.2 .2 & Resolved scale rate of strain \\
\hline$L_{s}$ & 3.2 .2 & $\begin{array}{c}\text { Smagorinsky-Lilly (S-L) mixing } \\
\text { length }\end{array}$ \\
\hline$\kappa$ & 3.2 .2 & von Kármán constant \\
\hline$D$ & 3.2 .2 & Nearest wall distance \\
\hline$C_{s}$ & 3.2 .2 & Smagorinsky constant \\
\hline$\Delta$ & 3.2 .2 & Local grid scale \\
\hline$u_{p, i}$ & 3.3.1 & Particle velocity \\
\hline$d_{p}$ & 3.3.1 & Particle diameter \\
\hline$C_{D}$ & 3.3 .1 & Particle drag coefficient \\
\hline$F_{x}$ & 3.3.1 & Additional particle forces \\
\hline$\rho_{p}$ & 3.3 .1 & Particle density \\
\hline$R e_{p}$ & 3.3 .1 & Particle Reynolds number \\
\hline$C_{d, \text { sphere }}$ & 3.3 .2 & Drag coefficient of a sphere \\
\hline$y$ & 3.3 .2 & Critical breakup term \\
\hline
\end{tabular}




\begin{tabular}{|c|c|c|}
\hline$C_{f}$ & 3.3 .2 & Dynamic drag oscillation constant \\
\hline$C_{b}$ & 3.3 .2 & Dynamic drag oscillation constant \\
\hline$C_{k}$ & 3.3 .2 & Dynamic drag oscillation constant \\
\hline$C_{d d}$ & 3.3.2 & Dynamic drag oscillation constant \\
\hline$\rho_{g}$ & 3.3.2 & Gas density \\
\hline$\rho_{l}$ & 3.3 .2 & Liquid density \\
\hline$\sigma$ & 3.3 .2 & Surface tension \\
\hline$\mu_{l}$ & 3.3.2 & Liquid dynamic viscosity \\
\hline$r$ & 3.3 .2 & Droplet radius \\
\hline$u_{i n j, \max }$ & 3.3 .3 & Maximum injection velocity \\
\hline$\Delta p_{\text {inj }}$ & 3.3 .3 & Injection pressure difference \\
\hline$C_{d}$ & 3.3.3 & Discharge coefficient \\
\hline$C_{a}$ & 3.3 .3 & Area coefficient \\
\hline$C_{v}$ & 3.3 .3 & Velocity coefficient \\
\hline$\dot{m}_{f}$ & 3.3 .3 & Mass flow rate \\
\hline$\dot{M}_{f}$ & 3.3.3 & Momentum flow rate \\
\hline$u_{\text {inj,ave }}$ & 3.3 .3 & Average injection velocity \\
\hline$L$ & 3.3.4 & Liquid core length \\
\hline$C_{L}$ & 3.3 .4 & Levich constant \\
\hline$d_{o}$ & 3.3.4 & Reference nozzle diameter \\
\hline$\omega$ & 3.3.4.1 & Perturbation growth rate \\
\hline$v_{l}$ & 3.3 .4 .1 & Liquid kinematic viscosity \\
\hline$\eta$ & 3.3.4.1 & Wave number \\
\hline$\Gamma$ & 3.3.4.1 & Perturbation wavelength \\
\hline
\end{tabular}




\begin{tabular}{|c|c|c|}
\hline$r_{o}$ & 3.3.4.1 & Undisturbed jet radius \\
\hline$I_{o}$ & 3.3.4.1 & First order Bessel function \\
\hline$I_{1}$ & 3.3.4.1 & First order Bessel function \\
\hline$K_{o}$ & 3.3.4.1 & Second order Bessel function \\
\hline$K_{1}$ & 3.3.4.1 & Second order Bessel function \\
\hline$Z$ & 3.3.4.1 & Ohnesorge number \\
\hline$T$ & 3.3.4.1 & Taylor number \\
\hline We & 3.3.4.1 & Weber number \\
\hline$r_{\text {child }}$ & 3.3.4.1 & Child droplet radius \\
\hline$\tau_{b u}$ & 3.3.4.1 & Characteristic breakup time \\
\hline$B_{o}$ & 3.3.4.1 & $\begin{array}{l}\text { Kelvin-Helmholtz breakup } \\
\text { constant }\end{array}$ \\
\hline$B_{1}$ & 3.3.4.1 & $\begin{array}{l}\text { Kelvin-Helmholtz breakup } \\
\text { constant }\end{array}$ \\
\hline$C_{3}$ & 3.3.4.2 & Rayleigh-Taylor breakup constant \\
\hline$\zeta^{*}$ & 3.4 & EDC turbulent length scale \\
\hline$\tau^{*}$ & 3.4 & EDC turbulent time scale \\
\hline$C_{\zeta}$ & 3.4 & EDC volume fraction constant \\
\hline$C_{\tau}$ & 3.4 & EDC time scale constant \\
\hline$Y_{k}^{*}$ & 3.4 & $\begin{array}{l}\text { Fine-scale mass fraction of species } \\
\qquad k\end{array}$ \\
\hline$\tau$ & 3.4.1 & Chemical ignition delay \\
\hline$\tau_{1}$ & 3.4 .1 & $1^{\text {st }}$ stage chemical ignition delay \\
\hline$\tau_{2}$ & 3.4 .1 & $2^{\text {nd }}$ stage chemical ignition delay \\
\hline$k_{q}$ & 3.4 .1 & Initiation rate \\
\hline
\end{tabular}




\begin{tabular}{|c|c|c|}
\hline$k_{p}$ & 3.4.1 & Product production rate \\
\hline$f_{1}$ & 3.4.1 & Shell model rate \\
\hline$f_{2}$ & 3.4.1 & Shell model rate \\
\hline$f_{3}$ & 3.4.1 & Shell model rate \\
\hline$f_{4}$ & 3.4.1 & Shell model rate \\
\hline$k_{b}$ & 3.4.1 & Branching rate \\
\hline$k_{t}$ & 3.4.1 & Termination rate \\
\hline$\chi$ & 3.4.1 & Product Ratio \\
\hline$[\mathrm{RH}]$ & 3.4.1 & Fuel molar concentration \\
\hline$[\mathrm{P}]$ & 3.4.1 & Product molar concentration \\
\hline [B] & 3.4.1 & $\begin{array}{l}\text { Branching species molar } \\
\text { concentration }\end{array}$ \\
\hline [Q] & 3.4.1 & $\begin{array}{l}\text { Intermediate species molar } \\
\text { concentration }\end{array}$ \\
\hline$[\mathrm{R}]$ & 3.4.1 & $\begin{array}{l}\text { Radical species molar } \\
\text { concentration }\end{array}$ \\
\hline [T] & 3.4.1 & $\begin{array}{l}\text { Termination species molar } \\
\text { concentration }\end{array}$ \\
\hline$k_{p 1}$ & 3.4.1 & Product production rate term \\
\hline$k_{p 2}$ & 3.4.1 & Product production rate term \\
\hline$k_{p 3}$ & 3.4.1 & Product production rate term \\
\hline$M W_{R}$ & 3.4.1 & Radical species molecular weight \\
\hline
\end{tabular}




\begin{tabular}{|c|c|c|}
\hline$M W_{C_{n} H_{2 m}}$ & 3.4.1 & Fuel molecular weight \\
\hline$M W_{O_{2}}$ & 3.4.1 & Oxygen molecular weight \\
\hline$M W_{B}$ & 3.4.1 & $\begin{array}{l}\text { Branching species molecular } \\
\text { weight }\end{array}$ \\
\hline$M W_{Q}$ & 3.4.1 & $\begin{array}{l}\text { Intermediate species molecular } \\
\text { weight }\end{array}$ \\
\hline$M W_{T}$ & 3.4.1 & $\begin{array}{c}\text { Terminating species molecular } \\
\text { weight }\end{array}$ \\
\hline$n$ & 3.4.1 & Fuel carbon number \\
\hline$m$ & 3.4.1 & Fuel hydrogen number \\
\hline$v_{i}^{\prime \prime}$ & 3.4.1 & $\begin{array}{l}\text { Product stoichiometric coefficient } \\
\text { for species } i\end{array}$ \\
\hline$v_{i}^{\prime}$ & 3.4.1 & $\begin{array}{l}\text { Reactant stoichiometric coefficient } \\
\text { for species } i\end{array}$ \\
\hline$k_{j}$ & 3.4.1 & Kinetic rate of reaction $j$ \\
\hline$[i]$ & 3.4.1 & Molar concentration of species $i$ \\
\hline
\end{tabular}




\subsection{Introduction}

Lean burning is an engine strategy that offers significant potential advantages over stoichiometric engines including: lower hydrocarbon, carbon dioxide, particulate and oxide emissions, higher thermal efficiencies, and lower fuel costs. For a variety of reasons though these potentials have never been completely realized, largely due to: ignition instabilities, incompatibility with emissions after-treatment systems, and difficulties in achieving acceptable performance over the entire load range. Solutions to these problems have been proposed but currently they primarily exist in research capacities. Prechamber combustion is one such solution that offers the possibility of achieving stable ignition while using lower than stoichiometric equivalence ratios. Effectively, the lean limit of combustion in internal combustion engines is extended, making lean burning engines more stable. Prechamber combustion though, is very complex as it incorporates more flow regimes than standard incylinder strategies, particularly the inter-chamber nozzle flow which may or may not cause local flame quenching. Thus CFD can potentially provide useful insight into the fluid processes occurring in these configurations.

As computing power steadily increases, greater simulation capability is possible in all forms of Computer Aided Engineering (CAE) but specifically within Computational Fluid Dynamics (CFD). Particularly challenging problems such as engine in-cylinder fluid processes are becoming tractable with greater potential to simulate turbulence and more detailed chemistry; however they are still generally time-consuming in comparison with product design cycles. Thus, physics simplification is still necessary in order to simulate these processes in a timely manner. However, greater physics capability can be 
incorporated and more insight can be gained into complex processes such as engine prechamber combustion than ever before, and greater analytical support can be provided to product designers. The main challenge of this work will be the incorporation of the various sub-models required to simulate in-cylinder processes to construct a model capable of predicting the pressure trace in a piston-prechamber configuration. Prechamber combustion is simulated in a piston configuration with the Euler-Lagrangian spray model, the Eddy-Dissipation Concept (EDC) model and the Shell ignition model tuned to model $\mathrm{C}_{10.17} \mathrm{H}_{19.91}$ fuel. Comparisons and insights are drawn between these processes when simulated with RANS and LES; greater capability is realized with LES. It is the purpose of this work to combine the aforementioned models with LES to demonstrate its capability and to present the tuned constants of $\mathrm{C}_{10.17} \mathrm{H}_{19.91}$ with the Shell model in order to produce a physics-reduced but practical engineering model of in-cylinder and prechamber processes. Chapter 2 reviews past prechamber designs and their operation along with previous modelling attempts. Chapter 3 summarizes all of the relevant mathematical models, while Chapter 4 presents the validation studies performed on the spray and ignition sub-models. Chapter 5 shows a demonstration of the final CFD model and Chapter 6 draws conclusions and recommends further work to improve the model. 


\subsection{Literature Review}

\subsection{In-Cylinder Modelling}

With the growing capabilities of computational simulations due to increases in computing power, modelling of problems encompassing more and more complex physics are becoming more commonplace. The challenge of modelling in-cylinder combusting flows with computational fluid dynamics (CFD) is one such problem. Piston engines typically have compressible, turbulent flows, either manifold or in-chamber fuel spray processes consisting of multi-phase physics and evaporation processes, either forced ignition or autoignition, low and high temperature combustion, complex modes of heat transfer and pollutant formation, all of which are occurring in a chamber of varying volume. To further increase the complexity, practical piston engines need to operate over a wide range of conditions, so the previously mentioned classes of physics occur and interact with each other in a daunting number of regimes. Significant progress has been made though since diesel engine simulations began to aid engine research in the late 1970s [1]. Both commercial and open-source CFD software packages have been developed with in-cylinder capabilities, the most commonly used being: KIVA, OpenFOAM, STAR-CD, FIRE, FLUENT and Vectis. Turbulence modelling for combustion purposes is beginning to develop from simplified Reyolds Averaged Navier-Stokes (RANS) to the more complex Large Eddy Simulation (LES) in practical in-cylinder configurations [2]. Several options for extending the applicability of the Euler-Lagrange multiphase model exist, and there exist several options for the treatment of both low and high temperature combustion ranging from modelling ten or fewer to thousands of chemical species [3-8]. Each of these sub-models 
requires validation with experimental data, and as mentioned before the interaction of these physical processes can be difficult to understand, such as the coupling of turbulence and chemistry. There currently does not exist an experimental setup that can be used to validate the interaction of all of these processes and thus isolated experiments must be used and conclusions combined to gain insights.

It is known that all in-cylinder processes are somewhat affected by turbulence and given its recent emergence into practicality, LES offers the modeler significant potential. LES effectively resolves the large scale flow structures and models the small scale structures, leading to a much greater overall resolution of any variable affected by turbulence when compared to RANS solutions. Often though, LES requires a much denser mesh than RANS, limiting its applicability and multiplying its computational cost. Researchers have recently shown that even with coarser meshes, the accuracy of LES predictions exceeds that of RANS [9-11]. LES also has a greater capability than RANS to model cycle-cycle variations, a commonly known process that occurs in piston engines [1213]. Furthermore, modellers often run in-cylinder cases with LES but without LES-specific sub models, such as spray and evaporation models [14]. This flexibility has the added benefit to the modeller that LES can be used without reformulating all of the other incylinder sub-models. It is generally regarded that LES-specific sub-models are required for the best results [1].

Generally speaking, there exists no general all-purpose chemistry model for modelling in-cylinder combustion processes. Several models and their variants exist that all have the capability of matching in-cylinder averaged pressure data reasonably well and 
their application is largely up to the user. The primary classifications of these models are determined by the way in which they couple chemistry and turbulence, and their treatment of chemical kinetics. It is well understood that the turbulent conditions within the combustion chamber greatly influence chemistry but a detailed description of this physical coupling is currently unknown to both experimenters and modellers. It is also known that the most accurate chemistry solutions can be obtained by modelling the largest number of chemical pathways. This approach however is computationally prohibitive and almost all models contain some strategy to reduce the kinetics.

The Eddy Dissipation Model (EDM) and it's extension, the Eddy Dissipation Concept (EDC) are models that assume a very strong relation between chemistry and turbulence and the chemical source terms are heavy functions of the turbulent mixing rate [15-17]. The EDC model has the added benefit that it is capable of solving both premixed and nonpremixed combustion and both infinitely-fast and finite-rate chemistry, making it attractive for modelling processes such as ignition, or slow forming species, such as $\mathrm{NO}_{\mathrm{x}}$. These models, however, are dependent on the accuracy of the turbulence parameters and an averaged turbulence method (RANS) will lead to averaged chemistry source terms (making phenomena such as local quenching or re-ignition difficult to model). Also, these models can incorporate kinetics of any level of detail with a conservation equation for each chemical species, so as the mechanism size increases, the EDC model becomes increasingly time consuming, both in terms of chemistry and CFD calculations.

The flamelet model for turbulent non-premixed combustion was developed with the assumption that chemical time scales are much shorter than the physical (mixing) time 
scales and thus, the chemistry can be decoupled from the fluid dynamics [18]. Implementation of the above assumption leads to the possibility of pre-calculated chemical source terms, which otherwise pose a significant challenge when considered simultaneously with the fluid equations. The flamelet model considers that a turbulent flame can be approximated with a discrete number of laminar flamelets. These flamelets are modelled with the traditional laminar counter-flow diffusion flame configuration where there exist separate streams of fuel and oxidizer that mix and form a flame front at some intermediate location [19-20], see Figure 1.

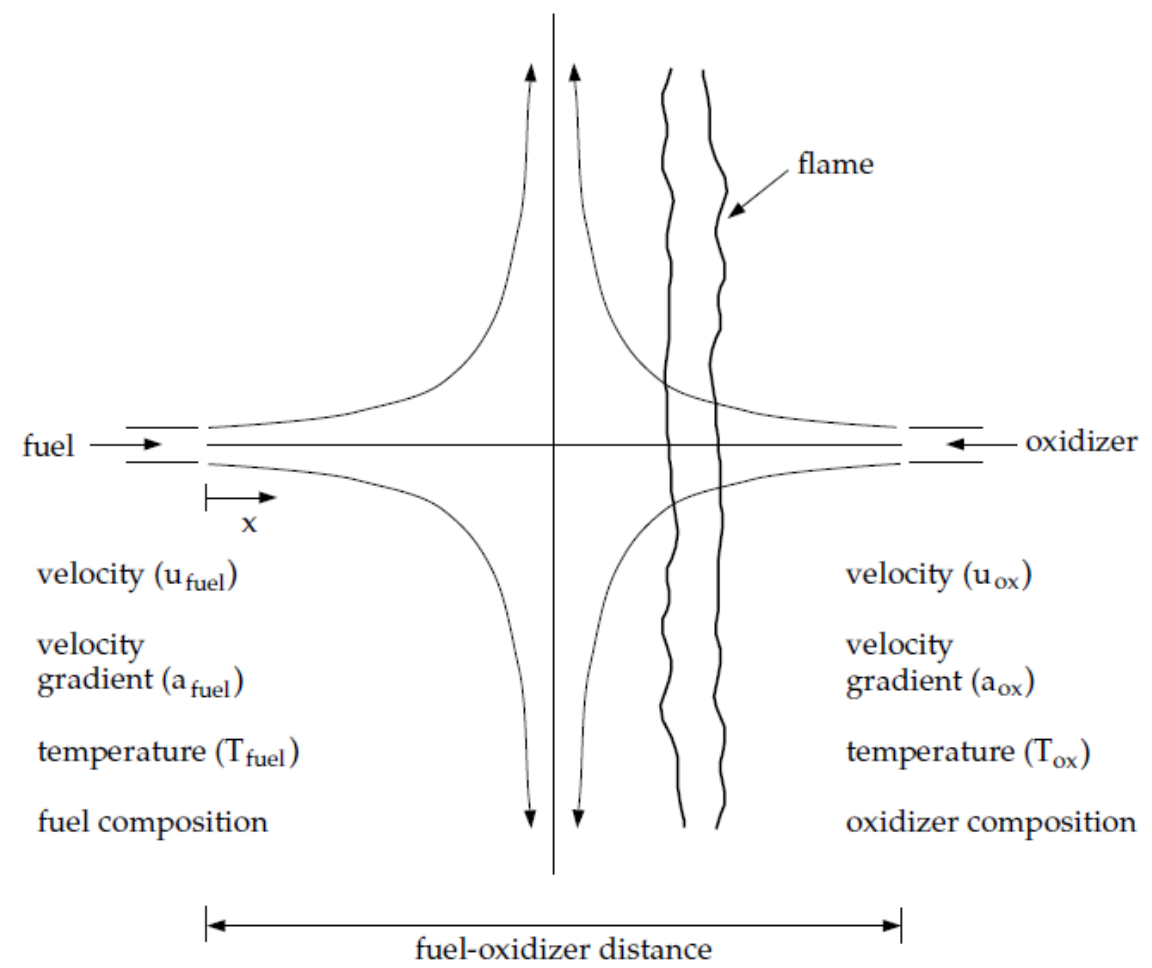

Figure 1 - Counter-flow diffusion flame [21].

The flamelet solution procedure is as follows: firstly, a library of flamelet variables (mass fractions, temperatures, reaction rates) is generated with a dedicated solver from 
scalar transport equations describing the laminar counter-flow diffusion flame. Secondly a Probability Density Function (PDF) is used to convert the laminar library variables into statistically determined turbulent variables for input into the CFD. Lastly, CFD is solved with two additional scalar conservation equations that serve as look-up parameters in the library. This scheme allows detailed chemistry to be solved while only adding two conservation equations, so the CFD computing time is kept low, although the onceprecomputed library and PDF generation time can still be high. The flamelet model as described so far is for steady-state systems. In cases that inherently need to be solved transiently, such as autoignition, re-ignition and extinction, the laminar flamelet model is insufficient. These phenomena all require a temporal resolution of residence times for ignition (representing the pre-ignition radical pool build-up) or extinction (representing the finite amount of time a flame needs to spend above a critical strain rate to be extinguished). The steady laminar flamelet model assumes that the flame responds immediately to the flow field and thus, no residence times are incorporated. Various unsteady flamelet models have been applied to diesel engine cases showing very good capability to predict in-cylinder pressures as well as reasonable pollutant predictions [2225]. These cases, however, require more complex models as well as large libraries or the assumption that only a single global strain rate exists for some time period. The Represetative Interactive Flamelet (RIF) and Flamelet Progress Variable (FPV) models do however represent significant steps towards modelling diesel engine combustion wherein the complete mechanism is incorporated. Further work has extended flamelet models to include the effect of liquid evaporation into the flamelet equations, thus further coupling the diesel processes by including the effect of the fuel evaporation within the libraries [26]. 
Another tabulation based method, the method of Intrinsic Low Dimensional Manifolds (ILDM) has recently been used to predict in-cylinder heat release and pressure [27-28]. The ILDM method, originally proposed by Maas and Pope is a mechanism reduction technique that decomposes the complete mechanism into fast and slow processes [29-30]. The main assumption with the method is that at all times, the state of the system is approaching a low-dimensional manifold which is governed by the ratecontrolling slow processes. Considerably less effort has been dedicated to practical applications of ILDM, as opposed to the efforts put towards improving the model with respect to laboratory flames. ILDM then remains primarily developmental, though it holds significant potential in modelling diesel engine combustion.

\subsection{Prechamber Review}

Lean burning engines pose several key advantages when compared to their stoichiometric counterparts. Firstly, when operated efficiently, less fuel is consumed and thus less $\mathrm{CO}_{2}$ is produced for a given power output. The higher specific heat ratio and higher allowable compression ratio both lead to an increase in thermal efficiency. Lean flames also burn at a lower temperature leading to less thermal $\mathrm{NO}_{\mathrm{x}}$ production and less heat loss to the walls. Despite these prospects, the concept is met with several difficulties. Firstly, the mixture ignitability becomes severely compromised as the equivalence ratio is reduced from stoichiometric, leading to engine misfires resulting in significant performance reduction. Secondly, as hydrocarbon-air equivalence ratios are lowered beyond stoichiometric, flame speed reduces. Lower flame speeds can result in the possibility of the flame not traversing the entire domain in the required time, leading to 
partial combustion, slow in-cylinder pressure rise, and high HC emissions. Furthermore, significant $\mathrm{NO}_{\mathrm{x}}$ reduction is only realized near the lean limit, nearest the region of the least reliable ignition.

Several enhanced ignition methods have been proposed to mitigate the difficulties associated with lean combustion, where the general concept is centered around increasing the ignition energy. Long duration sparks, multiple sparks per cylinder, plasma jet and laser igniters, and various divided chamber (prechamber) concepts are all methods designed to provide stable ignition in lean mixtures by increasing the ignition energy [31]. The multiple sparks per cylinder and prechamber concepts provide several ignition locations, negating the problem of slow flame propagation by increasing the size of the flame front.

Several types of prechambers exist, varying in fuel used, size, shape, fuel delivery, ignition source, and orifice size. The same basic principle is common to all prechambers though - ignition occurs in the prechamber (where there is a relatively easily ignitable mixture) - and the products from this process convect into the main chamber where a leaner mixture exists. The prechamber mixture is typically slightly richer than stoichiometric and is ignited by conventional means (spark or hot surface) and the main chamber mixture is typically lean. Given that the prechamber is smaller than the main chamber, the overall mixture is still lean. In these designs stable ignition is achieved and the active radicals expelled from the prechamber provide a stable homogeneous ignition source in the main chamber, which allows for fast combustion despite low flame speeds. 
Prechambers date back to 1918 with the 2-stroke Ricardo Dolphin engine (see Figure 2) which featured a fuel/air intake and a spark plug within the prechamber, which ignited a lean main chamber [32]. Many further attempts at the prechamber were made but none received widespread success until the Honda CVCC system in 1973 shown in Figure 3 [33]. Soon after, Toyota (Figure 4), Ford, Volkswagen, GM (Figure 5) and Porsche (Figure 6) developed their own comparable concepts [34-38]. However, despite their benefits (low engine-out $\mathrm{NO}_{\mathrm{x}}$, low fuel consumption and fast combustion [39]) prechambers were largely abandoned in the 1980's with the emergence of three-way catalytic converters which offered better emissions reduction. Three-way catalytic converters required stoichiometric combustion for optimal results and thus they were incompatible with prechambers.

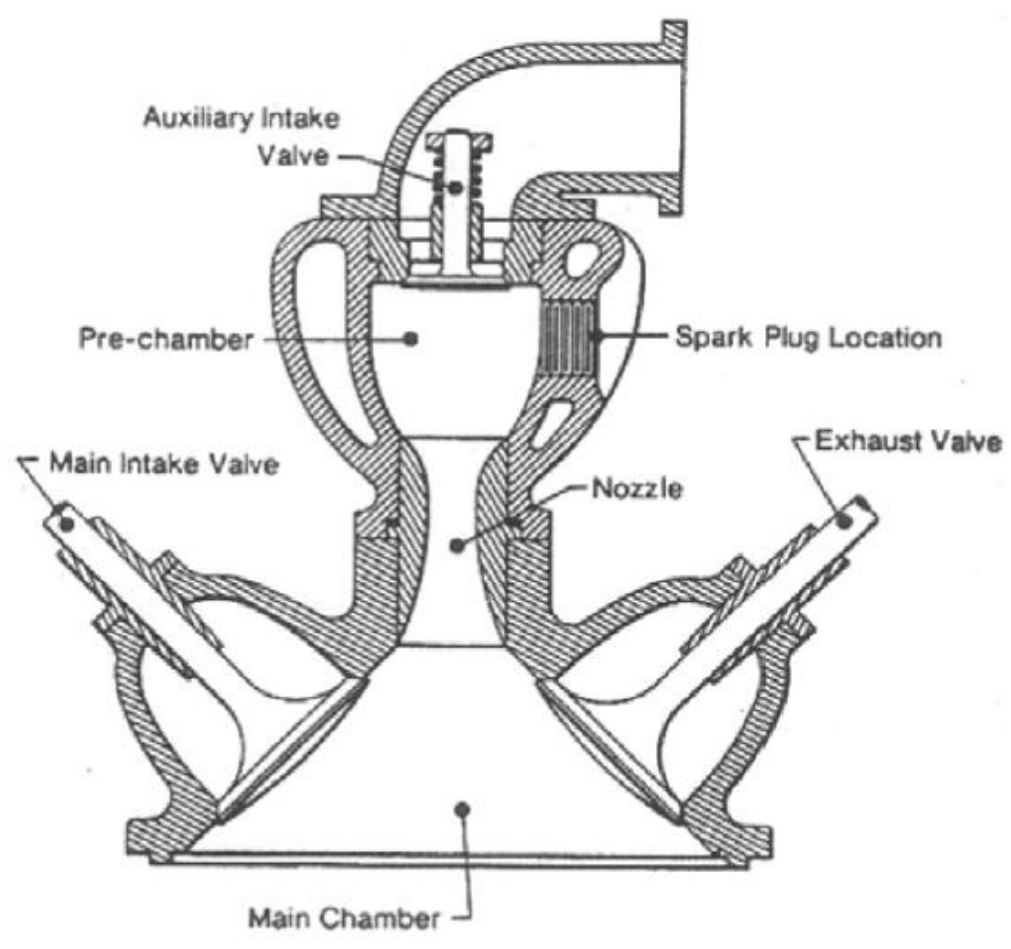

Figure 2 - Cylinder head and prechamber from Ricardo Dolphin engine [32]. 


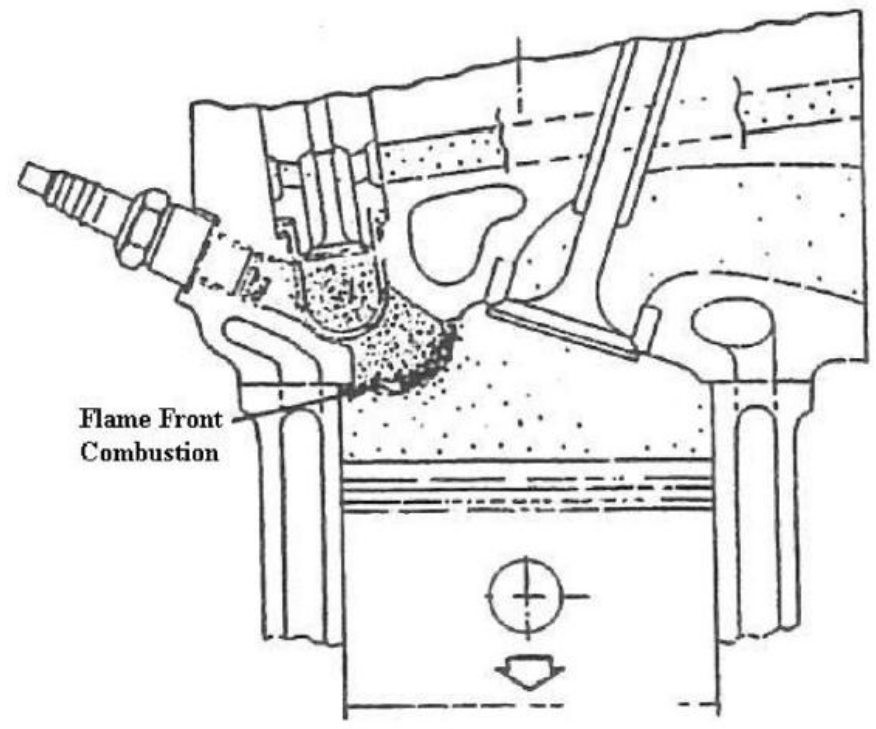

Figure 3 - Cylinder head and prechamber from Honda CVCC [33].

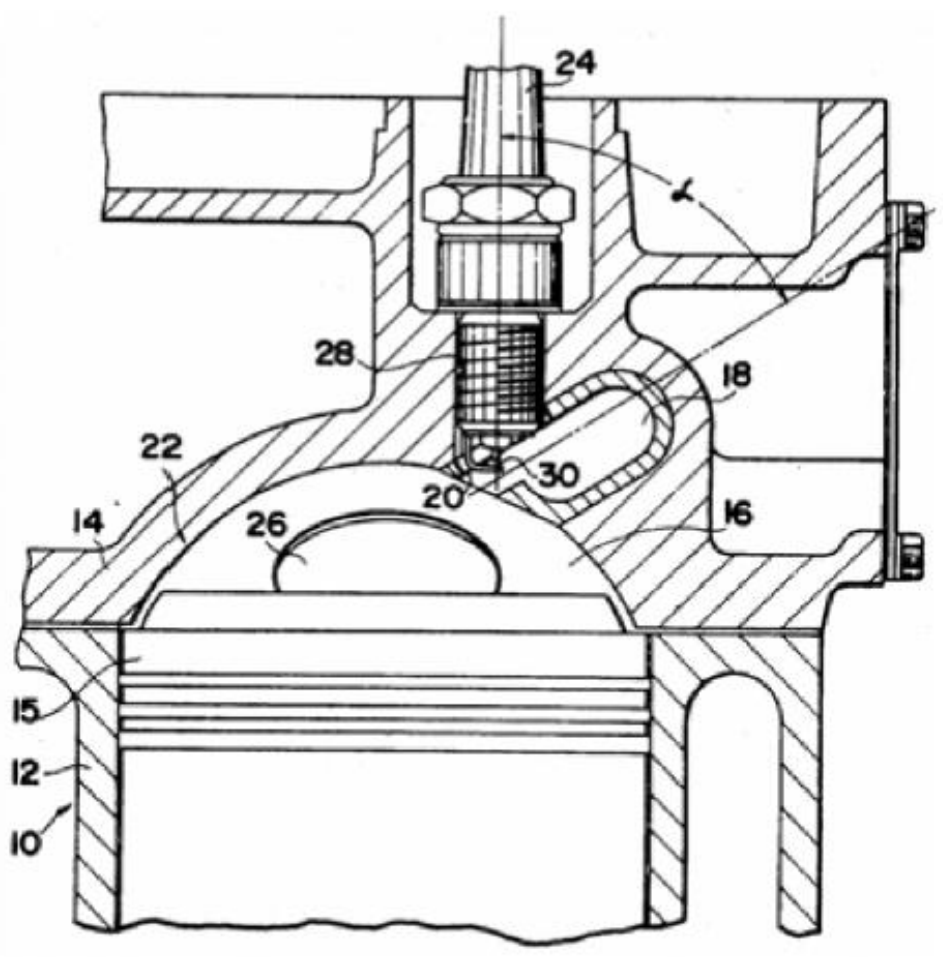

Figure 4-Cylinder head and turbulence generating pot from Toyota [34]. 


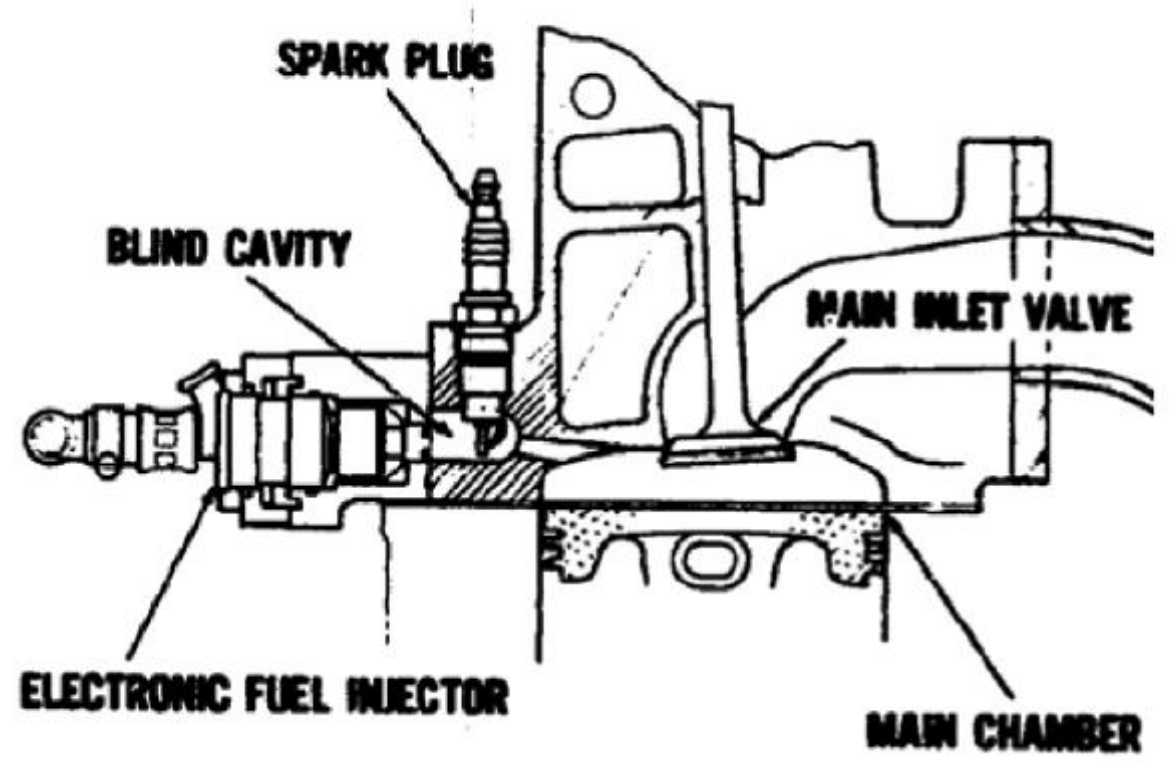

Figure 5 - Cylinder head and prechamber from GM [37].

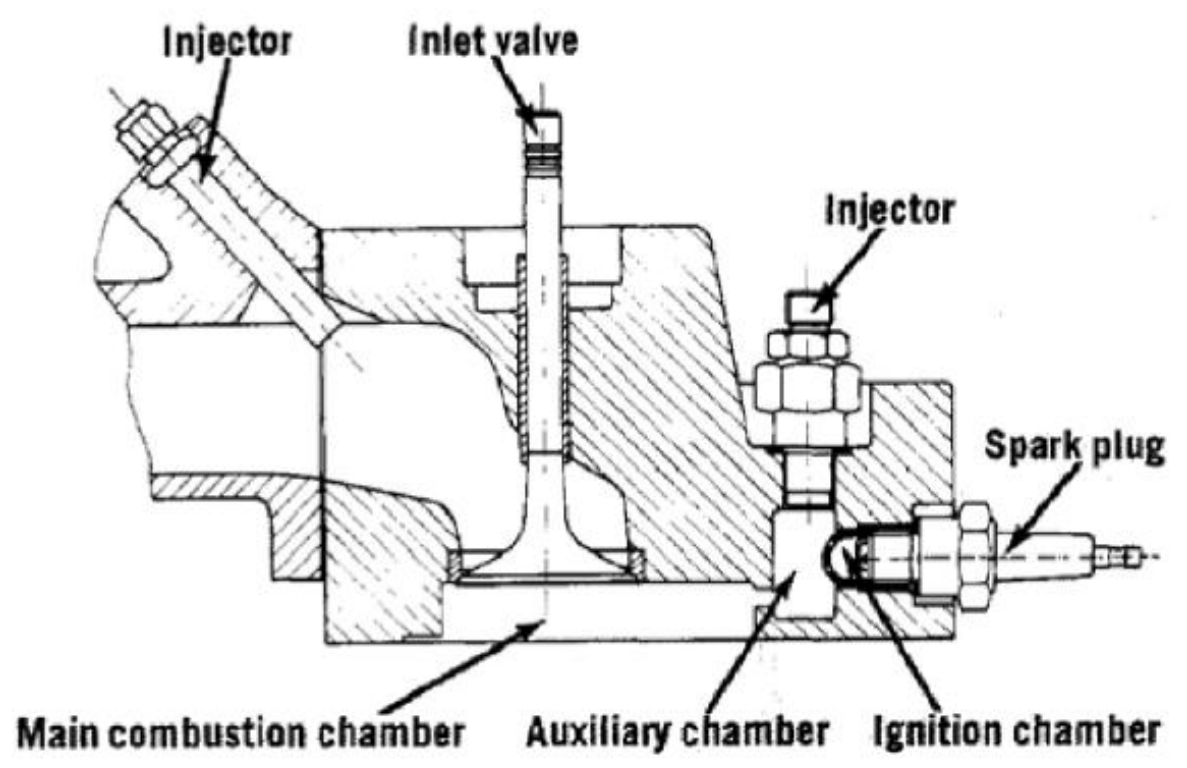

Figure 6-Cylinder head and auxiliary chamber from Porsche [38].

Many prechamber configurations have been explored in recent history albeit mostly in experimental configurations. These include developments of the LAG (Lavinia Aktivatisia 
Gorenia or Avalanche Activated Combustion) technique (where the orifice between chambers is reduced so that the flame from the prechamber is quenched, and only active radicals are expelled into the main chamber, Figure 7), dual-fuel configurations with hydrogen as the prechamber fuel, and dual-mode operation (high-load and part-load). For in-depth reviews of prechamber use the reader is referred to [31, 39-42].

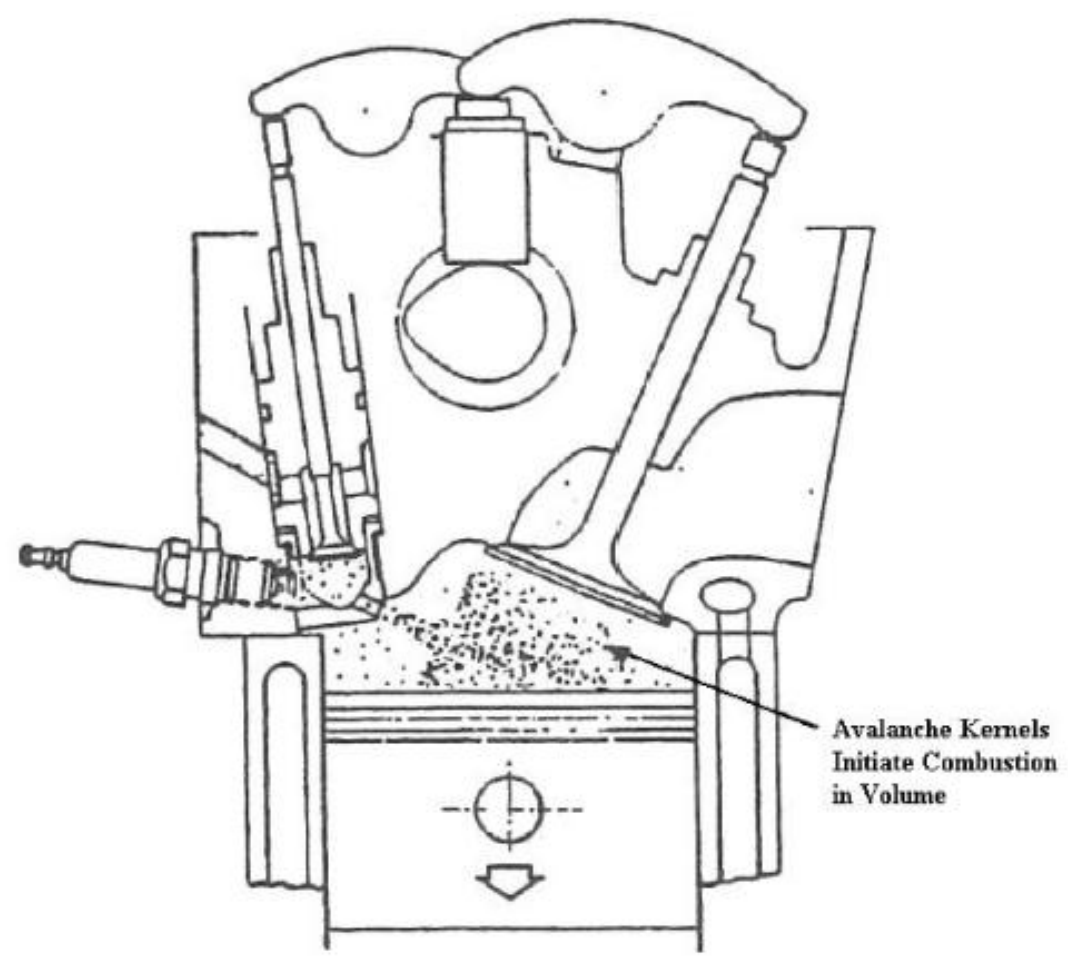

Figure 7 - Cylinder and prechamber demonstrating the LAG process [39].

As mentioned above, the primary reason for the slowed commercial application of prechambers are their incompatibility with current emissions after-treatment systems. Though, with fuel consumption again becoming an increasing concern, lean burning methods with suitable after-treatment may become a viable alternative to stoichiometric methods. 


\subsection{Previous Prechamber CFD Modelling}

In contrast to the large field dedicated to engine modelling with CFD, only a relatively small effort has been made to model prechambers, largely due to the fact that prechamber modelling is essentially a subset of engine modelling with additional chemical and physical complexity. The processes that need to be modelled are: the fuel injection (direct or indirect, single or multi-phase), ignition (forced or automatic), flame propagation (possibly with quenching and another ignition event as with the LAG process), and pollutant formation, as well as resolution of the mass, momentum, composition, energy and turbulence fields, all of which are inherently transient, with scarce experimental data for validation. Thus detailed CFD prechamber modelling is currently not possible, and as with standard diesel modelling, simplified techniques must be used.

Several prechamber-specific CFD cases have been reported in the past, primarily with the KIVA and FLUENT software packages. Of these cases it has been typical practice to use greatly reduced single-digit equation reaction mechanisms while $\mathrm{NO}_{\mathrm{x}}$, if modelled, has been modelled with a form of the Zeldovich mechanism [43-48]. More elaborate models using a dedicated multi-step ignition model or a larger mechanism have been used but considering current capabilities, the treatment of chemistry in prechamber-specific cases can be improved [49-50]. Another very notable feature of these cases is that they all use the $\mathrm{k}-\epsilon$ turbulence model, even in cold flow conditions [51-52]. It will be shown in the present work that the predictions of fuel liquid and vapour penetration in the FLUENT software package are better predicted with LES. For this reason it is justified to build a prechamberspecific case with the inclusion of LES to better account for the fuel injection and subsequent mixing process. 


\subsection{Theory}

\subsection{Governing Equations}

The software being used for this project is ANSYS Fluent, a finite volume computational fluid dynamics code based on solving the standard equations of mass, momentum and energy for compressible and incompressible flows. The compressible instantaneous forms of these equations are shown with Equations 1-3. Here, $\rho$ is the fluid density, $u_{i}$ is the fluid velocity in einsteinian notation, $\delta_{i j}$ is the kronecker-delta function, $p$ is the fluid pressure, $\mu$ is the dynamic or absolute viscosity, $\lambda$ is the second viscosity, usually taken to be $\lambda=-2 \mu / 3, g_{j}$ is the gravity force, $e$ is the internal energy and $q_{j}$ is the conductive heat flux.

$$
\begin{gathered}
\frac{\partial \rho}{\partial t}+\frac{\partial \rho u_{i}}{\partial x_{i}}=0 \\
\frac{\partial \rho u_{j}}{\partial t}+\frac{\partial \rho u_{j} u_{k}}{\partial x_{k}}=-\frac{\partial p}{\partial x_{j}}+\frac{\partial}{\partial x_{j}}\left(\lambda \frac{\partial u_{k}}{\partial x_{k}}\right)+\frac{\partial}{\partial x_{i}}\left[\mu\left(\frac{\partial u_{i}}{\partial x_{j}}+\frac{\partial u_{j}}{\partial x_{i}}\right)\right]+\rho g_{i} \\
\rho \frac{\partial e}{\partial t}+\rho u_{k} \frac{\partial e}{\partial x_{k}}=-p \frac{\partial u_{k}}{\partial x_{k}}+\lambda\left(\frac{\partial u_{k}}{\partial x_{k}}\right)^{2}+\mu\left(\frac{\partial u_{i}}{\partial x_{j}}+\frac{\partial u_{j}}{\partial x_{i}}\right) \frac{\partial u_{j}}{\partial x_{i}}-\frac{\partial q_{j}}{\partial x_{j}}
\end{gathered}
$$

Additionally, for chemically-reacting systems, where $N$ species exist, a conservation equation for $N-1$ species must be solved. The purpose of solving these equations is to determine the species composition at each location in the domain; $N-1$ equations are solved where the conservation equation for the most abundant species (usually $N_{2}$ ) is not solved. Solving the equations for the less abundant species reduces error as the mass 
fraction of the most abundant species can be deduced by summing the mass fractions of all species to unity. Equation 4 shows the expression for the conservation of species.

$$
\frac{\partial \rho Y_{k}}{\partial t}+\frac{\partial}{\partial x_{i}}\left[\rho\left(u_{i}+V_{k, i}\right) Y_{k}\right]=\dot{\omega}_{k}
$$

In the above equation, $Y_{k}$ is the mass fraction of species $k, V_{k, i}$ is the diffusive velocity of species $k$ in direction $i$, and $\dot{\omega}_{k}$ is the production/destruction rate of $k$. Along with the above equations, additional continuous phase equations must be solved for turbulence which will be discussed in the next section. If radiation and/or combustion is to be considered, additional equations need to be considered.

\subsection{Turbulence Models}

Reynolds Averaged Navier-Stokes (RANS) and Large Eddy Simulation (LES) are currently the most commonly used turbulence models in CFD. RANS modelling employs a strategy of averaging the turbulent fluctuations over a certain time, which blurs the transient nature of the turbulence but offers solutions in reasonable amounts of computational cost. LES is a strategy wherein the domain is filtered in a manner so that the large scale structures of the flow are resolved and the small scales of the flow are modelled. This strategy derives its validity in the notion that a large percentage of the flow energy transferred due to turbulence occurs as a result of the large scales of the flow. The large scales are also much more geometrically dependent, and are thus problem specific, whereas the small scales can be described somewhat independently of the flow geometry, leading to them being susceptible to tractable modelling. 


\subsubsection{Reynolds Averaged Navier-Stokes}

The basis of RANS modelling is that the parameters that fluctuate as a result of present turbulence are decomposed into their mean $\bar{\varphi}$ and fluctuating $\varphi^{\prime}$ components as shown in Figure 8.

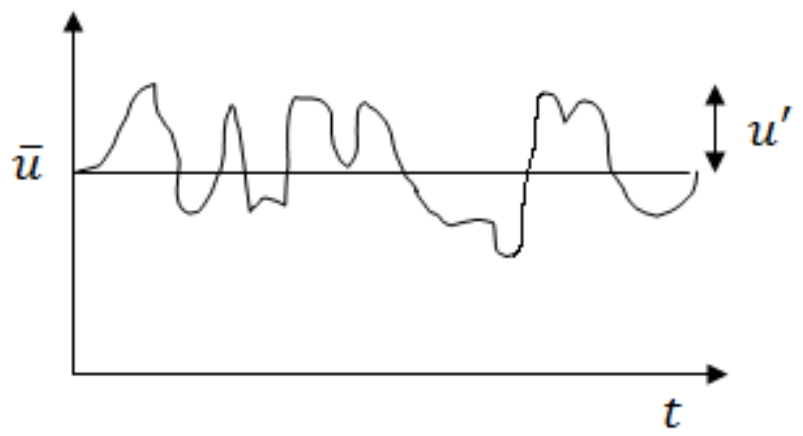

Figure 8 - Visual representation of the mean and fluctuating component of turbulent velocity.

Figure 8 shows a velocity, however, the decomposition can be done for any scalar such as temperature, pressure, or mass fraction. This strategy is shown in general form with Equation 5 where $\varphi$ is any decomposed variable.

$$
\varphi=\bar{\varphi}+\varphi^{\prime}
$$

With the above statistical decomposition defined, the Navier-Stokes equations can be re-written in terms of these new variables. This process will transform the instantaneous conservation equations into ensemble averaged equations. Only the conservation of momentum is shown here but the same process can be carried out for any conservation equation. Note that in Equation 6 the overbar was dropped (aside from the Reynolds stress term). 


$$
\frac{\partial}{\partial t}\left(\rho u_{i}\right)+\frac{\partial}{\partial x_{j}}\left(\rho u_{i} u_{j}\right)=-\frac{\partial p}{\partial x_{i}}+\frac{\partial}{\partial x_{j}}\left[\mu\left(\frac{\partial u_{i}}{\partial x_{j}}+\frac{\partial u_{j}}{\partial x_{i}}-\frac{2}{3} \delta_{i j} \frac{\partial u_{l}}{\partial x_{l}}\right)\right]+\frac{\partial}{\partial x_{j}}\left(-\rho \overline{u_{l}^{\prime} u_{\jmath}^{\prime}}\right)
$$

The end result is that an additional term named the Reynolds stress $\rho \overline{u_{\imath}^{\prime} u_{\jmath}^{\prime}}$ is added as shown above. This term in some form appears in every conservation equation aside from the conservation of mass, and must be modelled. The art of RANS turbulence modelling is then reduced to treatment of the Reynolds stress terms. Often additional conservation equations are required to solve for the Reynolds stresses and close the system of equations; these strategies are usually grouped by the number of conservation equations that they employ. With each additional conservation equation added, the complexity of the system increases by one more dimension and becomes harder to solve numerically. Thus the higher order turbulence models are thought of as being of a higher fidelity than the lower order models. Of the turbulence models considered in this report three will belong to the RANS family: Spalart-Allmaras (1 equation), Realizable k- $\varepsilon$ (2 equations) and the Reynolds Stress model (5 or 7 equations, depending if the problem is 2D or 3D).

In the case of most one and two-equation turbulence models, the last term in Equation 6 is made equal to the mean velocity gradients of the flow. This operation is called the Boussinesq approach and has proven to perform fairly well although it falsely assumes that the turbulent viscosity is an isotropic scalar [21].

$$
-\rho \overline{u_{\imath}^{\prime} u_{\jmath}^{\prime}}=\mu_{t}\left(\frac{\partial u_{i}}{\partial x_{j}}+\frac{\partial u_{j}}{\partial x_{i}}\right)-\frac{2}{3}\left(\rho k+\mu_{t} \frac{\partial u_{k}}{\partial x_{k}}\right) \delta_{i j}
$$


The varying strategies of solving Equation 7 are primarily focused on calculating the turbulent viscosity $\mu_{t}$.

\subsubsection{Realizable $k-\varepsilon$}

The $k-\varepsilon$ family and other two-equation models such as the $k-\omega$ model use the Boussinesq approximation, and an additional transport equation is solved for the turbulent kinetic energy $k$, and either the turbulent dissipation rate $\varepsilon$, or the specific dissipation rate $\omega$. The turbulent viscosity is then deduced as a function of $k$ and $\varepsilon$ or $k$ and $\omega$. The great advantage that two-equation models have over one-equation models is that they can compute both the turbulent velocity and the turbulent length scales, thus they are vastly more applicable to flows with variances in length scales.

The realizable $k-\varepsilon$ model was chosen in this study due to its ability to accurately predict the spreading rate of round jets, a prominent feature for a conical spray. The transport equations for $k$ and $\varepsilon$ are shown below [21]:

$$
\begin{gathered}
\frac{\partial}{\partial t}(\rho k)+\frac{\partial}{\partial x_{j}}\left(\rho k u_{j}\right)=\frac{\partial}{\partial x_{j}}\left[\left(\mu+\frac{\mu_{t}}{\sigma_{k}}\right) \frac{\partial k}{\partial x_{j}}\right]+G_{k}+G_{b}-\rho \varepsilon-Y_{M}+S_{k} \\
\frac{\partial}{\partial t}(\rho \varepsilon)+\frac{\partial}{\partial x_{j}}\left(\rho \varepsilon u_{j}\right)=\frac{\partial}{\partial x_{j}}\left[\left(\mu+\frac{\mu_{t}}{\sigma_{\varepsilon}}\right) \frac{\partial \varepsilon}{\partial x_{j}}\right]+\rho C_{1} S_{\varepsilon}-\rho C_{2} \frac{\varepsilon^{2}}{k+\sqrt{v \varepsilon}}+C_{1 \varepsilon} \frac{\varepsilon}{k} C_{3 \varepsilon} G_{b}+S_{\varepsilon} \\
C_{1}=\max \left[0.43, \frac{\eta}{\eta+5}\right] \\
\eta=S \frac{k}{\varepsilon} \\
S=\sqrt{2 S_{i j} S_{i j}}
\end{gathered}
$$




$$
S_{i j}=\frac{1}{2}\left(\frac{\partial u_{j}}{\partial x_{i}}+\frac{\partial u_{i}}{\partial x_{j}}\right)
$$

Where $G_{k}$ and $G_{b}$ are the production of $k$ from mean velocity gradients and buoyancy respectively. $Y_{M}$ is the fluctuating dilatation in the compressible turbulence contribution to $\varepsilon . \sigma_{k}$ and $\sigma_{\varepsilon}$ are the turbulent Prandtl numbers for $k$ and $\varepsilon . S_{k}$ and $S_{\varepsilon}$ are additional source terms and $C_{2}$ and $C_{3 \varepsilon}$ are model constants. The turbulent viscosity is calculated as follows, in other versions of the $k-\varepsilon$ model the term $C_{\mu}$ is treated as a constant, whereas in the realizable model it is treated in the following manner [21]:

$$
\begin{gathered}
\mu_{t}=\rho C_{\mu} \frac{k^{2}}{\varepsilon} \\
C_{\mu}=\frac{1}{A_{o}+A_{s}\left(\frac{k U^{*}}{\varepsilon}\right)} \\
A_{s}=\sqrt{6} \cos \phi \\
\phi=\frac{1}{3} \cos ^{-1}(\sqrt{6} W) \\
W=\frac{S_{i j} S_{j k} S_{k i}}{S^{3}} \\
U^{*}=\sqrt{S_{i j} S_{i j}+\widetilde{\Omega}_{i j} \widetilde{\Omega}_{i j}} \\
\widetilde{\Omega}_{i j}=\overline{\Omega_{\imath \jmath}}-3 \varepsilon_{i j k} \omega_{k}
\end{gathered}
$$

Here, $A_{o}$ is a constant equal to $4.04, \overline{\Omega_{l \jmath}}$ is the mean rate of the rotation tensor viewed in a rotating reference frame with angular velocity $\omega_{k}$. 


\subsubsection{Large Eddy Simulation}

In terms of resolution of the flow, RANS methods model all flow scales, while in contrast, Direct Numerical Simulation (DNS) resolves all scales of the flow and approximates nothing. LES falls in between these two strategies as it resolves the large flow scales and models the small scales with the use of a filtering process. The large scales of a flow are known to be highly anisotropic and geometrically dependent while the small scales are generally more isotropic. LES exploits this fact in an attempt to provide solutions of a higher order than that with RANS, without the significantly prohibitive constraints of DNS. In an analogous procedure to RANS, the Navier-Stokes equations are filtered instead of averaged. The filtering process separates the large and small flow scales and the end result is transport equations for the large scales of the flow with additional terms governing the small scales that must be modelled. A general filtered variable is formed as shown in Equation 21, where $G\left(\boldsymbol{x}, \boldsymbol{x}^{\prime}\right)$ is the filter function that determines how the scales of the flow are separated.

$$
\bar{\phi}(\boldsymbol{x})=\int \phi\left(\boldsymbol{x}^{\prime}\right) G\left(\boldsymbol{x}, \boldsymbol{x}^{\prime}\right) d \boldsymbol{x}^{\prime}
$$

The momentum equation takes the following form after being filtered, where $\tau_{i j}$ is the subgrid stress also shown below [21].

$$
\frac{\partial}{\partial t}\left(\rho \bar{u}_{i}\right)+\frac{\partial}{\partial x_{j}}\left(\rho \bar{u}_{i} \bar{u}_{j}\right)=\frac{\partial}{\partial x_{j}}\left[\mu\left\{\frac{\partial \bar{u}_{i}}{\partial x_{j}}+\frac{\partial \bar{u}_{j}}{\partial x_{i}}\right\}-\frac{2}{3} \mu \frac{\partial \bar{u}_{l}}{\partial x_{l}} \delta_{i j}\right]-\frac{\partial \bar{p}}{\partial x_{i}}-\frac{\partial \tau_{i j}}{\partial x_{j}}
$$

The primary modelling options when using LES are then in terms of the choice of the filtering function $G$ and the manner in which the sub-grid stresses $\tau_{i j}$ are modelled. 
Equations 23 and 24 show the form of $\tau_{i j}$ for incompressible and compressible flows, respectively.

$$
\begin{aligned}
& \tau_{i j, \text { incomp }}=\rho \overline{u_{\imath} u_{\jmath}}-\rho \bar{u}_{i} \bar{u}_{j} \\
& \tau_{i j, \text { comp }}=\bar{\rho} \widetilde{u_{\imath} u_{J}}-\bar{\rho}_{i} \tilde{u}_{i} \tilde{u}_{j}
\end{aligned}
$$

For incompressible flows, the Boussinesq approximation can be used to compute the sub-grid stresses as shown below, where $\mu_{s g s}$ is the sub-grid turbulent viscosity and $\bar{S}_{i j}$ is the strain rate term for the resolved scale.

$$
\begin{gathered}
\tau_{i j}-\frac{1}{3} \tau_{k k} \delta_{i j}=-2 \mu_{s g s} \bar{S}_{i j} \\
\bar{S}_{i j}=\frac{1}{2}\left(\frac{\partial \bar{u}_{i}}{\partial x_{j}}+\frac{\partial \bar{u}_{j}}{\partial x_{i}}\right)
\end{gathered}
$$

For compressible flows, Equation 25 is split into its deviatoric and isotropic components; the deviatoric component is then modelled with the following Smagorinksy model [21].

$$
\begin{gathered}
\tau_{i j, c o m p}=\left[\tau_{i j}-\frac{1}{3} \tau_{k k} \delta_{i j}\right]_{d e v}+\left[\frac{1}{3} \tau_{k k} \delta_{i j}\right]_{i s o} \\
{\left[\tau_{i j}-\frac{1}{3} \tau_{k k} \delta_{i j}\right]_{d e v}=2 \mu_{s g s}\left(S_{i j}-\frac{1}{3} \tau_{k k} \delta_{i j}\right)}
\end{gathered}
$$

Several models exist for the prediction of $\mu_{s g s}$. This report will consider solely the Smagorinsky-Lilly (S-L) model [21]. The S-L model uses the resolved velocity scales and local mixing length to determine the turbulent viscosity. Equations 29-31 show the makeup 
of the S-L model where $L_{s}$ is the mixing length, $\kappa$ is the von Kármán constant, $d$ is the nearest wall distance, $C_{S}$ is the Smagorinksy constant and $\Delta$ is the local grid scale.

$$
\begin{gathered}
\mu_{s g s}=\rho L_{s}^{2}|\bar{S}| \\
L_{s}=\min \left(\kappa d, C_{s} \Delta\right) \\
|\bar{S}|=\sqrt{2 \bar{S}_{i j} \bar{S}_{i j}}
\end{gathered}
$$

\subsection{Spray Model}

Liquid injections into a gaseous environment occur in a wide number of engineering applications, among the most common of these is the injection of liquid fuel for the purpose of combustion. Depending on the application, these injections occur at a wide variety of operating conditions with a range of fuels. Understanding of these processes is thus crucial in effective and efficient application, especially considering the well-known high dependence that combustion behaviour has on fuel-air mixing. Several methods exist to predict spray behaviour in a 0 or 1 dimensional sense, usually focused on predictions of spray length, spray angle or Sauter mean diameter (SMD) [53-55]. Recently however, CFD has been gaining applicability in modelling sprays in three-dimensional transient environments $[8,56]$. The three primary methods of modelling multiphase flows within CFD are the Euler-Lagrange, Euler-Euler and Volume of Fluid (VOF) methods. For dilute sprays, as fuel injection processes are generally approximated, the Euler-Lagrangian method is the most popular. 
Given the complexity of the governing physics in spray processes, the associated CFD modelling is still considered to be in developmental stages. No singular general model or method exists for accurate prediction of spray behaviour, therefore a sizeable amount of effort is required when attempting to model a spray. A spray's development is widely known to be a function of: nozzle state, nozzle geometry, fuel used, rail conditions, ambient gas conditions, and turbulence. Modelling the entirety of this problem is extremely difficult and only generally attempted with Direct Numerical Simulation (DNS), which is computational prohibitive in almost all cases.

Practical modelling applications are thus limited to relatively large reductions in physical complexity at the benefit of achieving a useful result in a reasonable time [57-61]. In this vein, much spray work simplifies the injection, breakup, and turbulent dispersion processes in several ways.

The Euler-Euler model uses an Eulerian framework to simulate each phase as its own continuum while the Euler-Lagrange method uses an Eulerian method for the continuous bulk phase and a Lagrangian method for the dispersed phase. In applications where the volume fraction of both phases is significant (such as if each phase is present in comparable concentrations) the Euler-Lagrangian model is invalid [21]. In cases where the secondary phase represents a small fraction of the domain's volume (such as dilute applications) the Euler-Lagrange method is much more appropriate [21]. It is common practice in spray modelling to assume that the liquid spray occupies a small portion of the domain's volume and to use a Euler-Lagrange model, namely the discrete phase model. The low volume fraction assumption is only strictly true for regions on the periphery or 
downstream of the spray where significant spreading of the liquid has occurred. In regions near the nozzle, the domain almost entirely comprises liquid and a Lagrangian method is not appropriate. In recent developments, hybrid models have been proposed that use an Eulerian framework to model the near-nozzle portion of the spray that interfaces with a Lagrangian framework in more dilute regions of the spray [8]. In this study the standard discrete phase model is used for simplicity.

\subsubsection{Particle Motion}

The discrete phase model interfaces with the conservation equations of mass, momentum, energy and species with a particle force balance in order to couple the continuous gas and liquid droplet phases. The force balance and appropriate equations are

listed below [21]. $u_{p, i}$ is the particle velocity, $d_{p}$ is the particle diameter, $C_{D}$ is the drag coefficient and subscript $p$ stands for particle. $F_{x}$ represents a number of additional contributions to particle accelerations for special cases, these forces being: thermophoretic forces, Brownian forces, Saffman's lift forces, etc. In most cases these additional forces are negligible and $F_{x}$ is ignored.

$$
\begin{gathered}
\frac{d u_{p, i}}{d t}=F_{D}\left(u_{i}-u_{p}\right)+\frac{g_{x}\left(\rho_{p}-\rho\right)}{\rho_{p}}+F_{x} \\
F_{D}=\frac{18 \mu}{\rho_{p} d_{p}^{2}} \frac{C_{D} R e_{p}}{24} \\
R e_{p}=\frac{\rho d_{p}\left|u_{p}-u\right|}{\mu}
\end{gathered}
$$




\subsubsection{Particle Drag Laws}

In order to account for the drag on the droplet, a dynamic model was used wherein the droplet is assumed to oscillate between a sphere and a disk. The actual shape of the particles is very complex and consists of several modes of oscillation, the sphere-disk assumption only considers the first mode. The drag coefficient is determined for a sphere as a function of particle Reynolds number and then an additional equation is used to determine the droplets deviation from a spherical shape. $C_{d, \text { sphere }}$ is computed as shown below with Equation 35, and the deviation from a sphere is found with Equations 36 and 37. $y$ is the parameter that tracks the shape of the particle, $y=0$ gives a drag coefficient of a sphere while $y=1$ gives a drag coefficient of a disk.

$$
\begin{gathered}
C_{d, \text { sphere }}=\left\{\begin{array}{cc}
0.424 & R e_{p}>1000 \\
\frac{24}{R e_{p}}\left(1+\frac{1}{6} R e_{p}^{2 / 3}\right) & R e_{p} \leq 1000
\end{array}\right. \\
C_{D}=C_{d, \text { sphere }}(1+2.632 y) \\
\frac{d^{2} y}{d t^{2}}=\frac{C_{f}}{C_{b}} \frac{\rho_{g}}{\rho_{l}} \frac{u_{p}^{2}}{r^{2}}-\frac{C_{k} \sigma}{\rho_{l} r^{3}} y-\frac{C_{d d} \mu_{l}}{\rho_{l} r^{2}} \frac{d y}{d t}
\end{gathered}
$$

\subsubsection{Droplet Injection}

The well-known and numerically efficient injection method known as the blob method was used in this study [62]. The blob method assumes that the liquid jet emerging from the nozzle interface can be approximated with large droplets of a diameter of the same order as the nozzle hole diameter. This method is favourable when used with the Euler-Lagrangian framework because the entire liquid domain can be simulated with discrete droplets. If slug flow is assumed in the nozzle then the emerging blob diameter will 
equal the nozzle hole diameter. Realistically however, in diesel engine applications the nozzle is often in a cavitating state and a discharge coefficient is used to model the effective flow area of the nozzle. Figure 9 shows an illustration of the approximation that the blob method employs to model the liquid core, and Figure 10 shows what the blob method actually does. Note that when the blob method is used, there may be some approximate methods to account for primary breakup but essentially only secondary breakup is simulated.

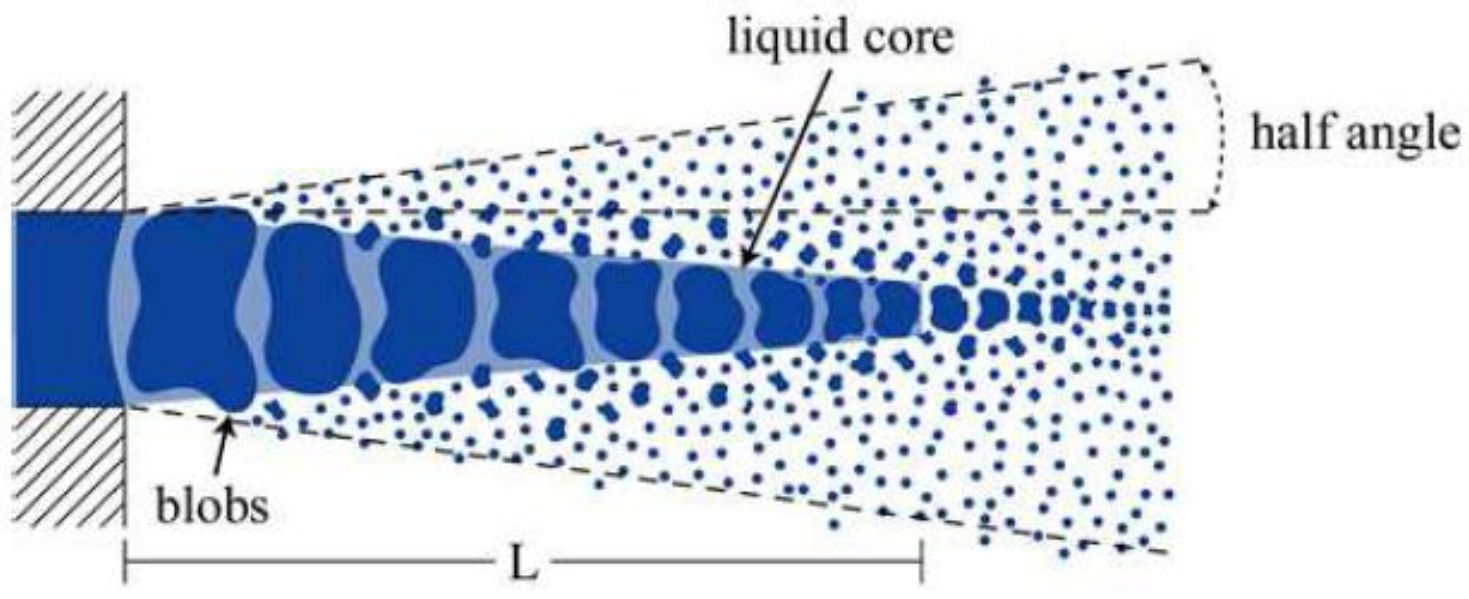

Figure 9 - Blob method approximation of the liquid core [21].

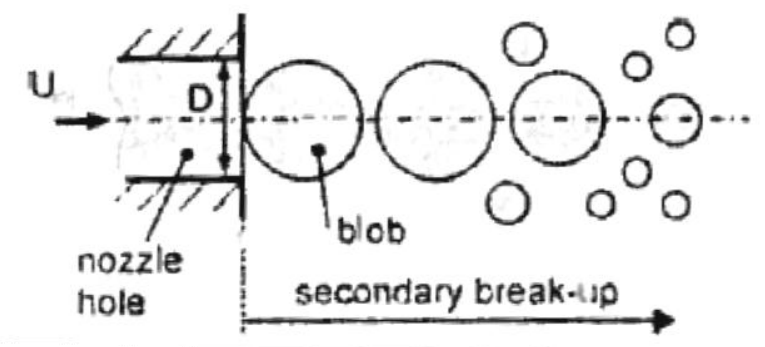

Figure 10 - CFD illustration of injected droplet size [62]. 
A significant drawback of the blob method is that the velocities of the injected droplets are hard to determine. The theoretical maximum injection velocity can be determined from applying the Bernoulli equation across the injector. The actual injection velocity has been reported to be within 70-90\% of this maximum velocity [63-65].

$$
u_{i n j, \max }=\sqrt{\frac{2 \Delta p_{i n j}}{\rho_{l}}}
$$

Several methods exist to predict the injection velocity when cavitation is taken into account. The following method uses a discharge coefficient $C_{d}$ as a function of an area coefficient $C_{a}$ and a velocity coefficient $C_{v}$ to determine the injector mass flow rate $\dot{m}_{f}$, momentum flow rate $M_{f}$ and average injection velocity $u$ [66-67].

$$
\begin{gathered}
C_{d}=C_{a} C_{v} \\
\dot{m}_{f}=C_{a} A \rho_{f} C_{v} u_{i n j, \max } \\
\dot{M}_{f}=\dot{m}_{f} C_{v} u_{\text {inj,max }} \\
C_{a}=\frac{2 A C_{d}^{2} \Delta p_{\text {inj }}}{\dot{M}_{f}} \\
u_{i n j, a v e}=C_{v} u_{i n j, \max }
\end{gathered}
$$

\subsubsection{Breakup Models}

In a typical diesel spray, a wide range of breakup modes occur at various locations in the spray. For this reason, combination breakup models have been developed to better 
predict the different classes of droplet breakup. The combined method for full cone sprays used here is the Kelvin-Helmholtz-Rayleigh-Taylor (KH-RT) model. It has been determined that in the near nozzle region of the spray, the KH model behaviour dominates while the RT model better predicts breakup outside of the liquid core. The boundary between the regions where each model is used is the liquid core length $L$ (See Figure 11), shown with Equation 44. $C_{L}$ is the Levich constant and $d_{o}$ is a reference nozzle diameter.

$$
L=C_{L} d_{o} \sqrt{\frac{\rho_{l}}{\rho_{g}}}
$$

Figure 11 shows the regions in which each model is favoured. In Fluent only $\mathrm{KH}$ breakup is used in the liquid core and both $\mathrm{KH}$ and RT are used in a competing manner in the region outside of the core.

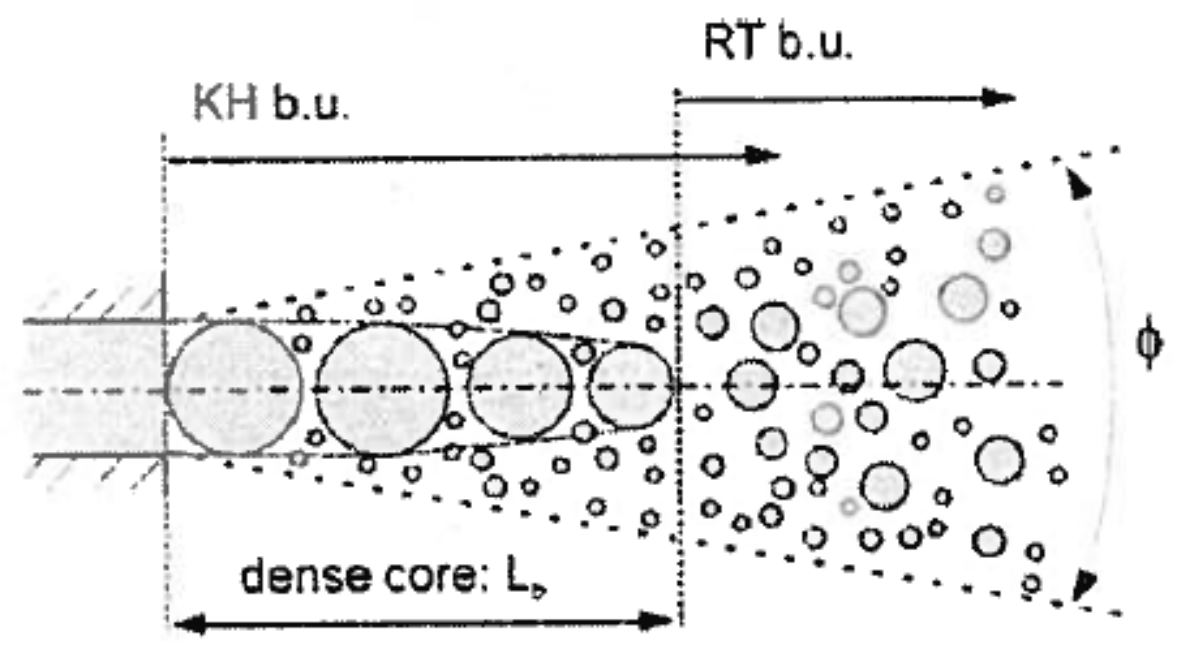

Figure 11 - Illustration of breakup model regions [62]. 


\subsubsection{Kelvin-Helmholtz Breakup Model}

The KH model is based on a first-order linear analysis of a cylindrical liquid jet immersed into a stationary gas. Both phases are assumed to be incompressible and the gas is assumed inviscid. It is also assumed that the liquid-gas interface exists in a sinusoidal form. Due to the relative motion between liquid and gas, these waves grow to a condition where jet breakup occurs. After converting the Navier-Stokes equations for each phase into stream and potential functions, a dispersion equation can be determined, which relates the growth rate of a perturbation to its wavelength [62].

$$
\begin{gathered}
\omega^{2}+2 v_{l} \eta^{2} \omega\left(\frac{I_{1}^{\prime}\left(\eta r_{o}\right)}{I_{o}\left(\eta r_{o}\right)}-\frac{2 \eta l}{\eta^{2}+l^{2}} \frac{I_{1}\left(\eta r_{o}\right)}{I_{o}\left(\eta r_{o}\right)} \frac{I_{1}^{\prime}\left(l r_{o}\right)}{I_{1}\left(l r_{o}\right)}\right) \\
=\frac{\sigma \eta}{\rho_{l} r_{o}^{2}}\left(1-r_{o}^{2} \eta^{2}\right)\left(\frac{l^{2}-\eta^{2}}{l^{2}+\eta^{2}}\right) \frac{I_{1}\left(\eta r_{o}\right)}{I_{o}\left(\eta r_{o}\right)} \\
+\frac{\rho_{g}}{\rho_{l}}\left(u_{r e l}-\frac{i \omega}{\eta}\right)^{2} \eta^{2}\left(\frac{l^{2}-\eta^{2}}{l^{2}+\eta^{2}}\right) \frac{I_{1}\left(\eta r_{o}\right) K_{o}\left(\eta r_{o}\right)}{I_{o}\left(\eta r_{o}\right) K_{1}\left(\eta r_{o}\right)} \\
\eta=\frac{2 \pi}{\Gamma} \\
l^{2}=\eta^{2}+\frac{\omega}{v_{l}}
\end{gathered}
$$

where $\omega$ is the perturbation growth rate, $v_{l}$ is the liquid kinematic viscosity, $\eta$ is the wave number, $\Gamma$ is the perturbations wavelength, $\sigma$ is the liquids surface tension, $r_{o}$ is the undisturbed jet radius, $I_{o}$ and $I_{1}$ are first order modified Bessel functions and $K_{o}$ and $K_{1}$ are second order Bessel functions. Analysis of Equation 45 shows that for some wave number 
there exists a single maximum growth rate. This maximum growth rate represents the greatest instability on the jet and it will cause breakup [68]. From Equation 45:

$$
\begin{aligned}
& \omega_{\max }\left[\frac{\rho_{l} r_{o}^{3}}{\sigma}\right]^{0.5}=\frac{0.34+0.38 W e_{g}^{1.5}}{(1+Z)\left(1+1.4 T^{0.6}\right)} \\
& \frac{\eta_{\max }}{r_{o}}=9.02 \frac{\left(1+0.45 Z^{0.5}\right)\left(1+0.4 T^{0.7}\right)}{\left(1+0.865 W e_{g}^{1.67}\right)^{0.6}}
\end{aligned}
$$

$Z, T$ and $W e$ are the Ohnesorge, Taylor and Weber numbers shown below.

$$
\begin{gathered}
Z=\frac{\mu}{\sqrt{\rho_{l} r_{o} \sigma}} \\
T=Z \sqrt{W e_{g}} \\
W e_{g}=\frac{\rho_{g} r_{o} u_{r e l}^{2}}{\sigma}
\end{gathered}
$$

With the above equations, expressions for the child droplet radius can be obtained and are shown below. The rate of parent droplet radius decrease is also shown where $\tau_{b u}$ is a characteristic break-up time.

$$
\begin{gathered}
r_{\text {child }}=B_{o} \eta_{\max } \\
\frac{d r}{d t}=-\frac{r-r_{\text {child }}}{\tau_{b u}} \\
\tau_{b u}=3.788 B_{1} \frac{r}{\eta_{\max } \omega_{\max }}
\end{gathered}
$$


$B_{o}$ is a constant equalling 0.61 and $B_{1}$ is a constant subject to tuning. $B_{1}$ is thought to contain effects of the nozzle geometry and has been used in the range of 1-60.

\subsubsection{Rayleigh-Taylor Breakup Model}

Like the KH model, the Rayleigh-Taylor (RT) model is based on a stability analysis of a liquid-gas interface subject to surface perturbations. The state of the system is assumed stable if the gas accelerates into the liquid but unstable if the liquid accelerates into the gas. Breakup occurs if the aerodynamic force from the acceleration is large enough. The dispersion equations were then derived with respect to the acceleration $a$ of the droplet; first the drag on the drop is derived and then it is divided by the droplet mass to find the acceleration.

$$
\begin{gathered}
D=\frac{1}{2} \rho_{g} u_{r e l}^{2} \pi r^{2} C_{D} \\
a=\frac{3}{8 r} \frac{\rho_{g}}{\rho_{l}} u_{r e l}^{2} C_{D}
\end{gathered}
$$

Again, as with the $\mathrm{KH}$ model, expressions are found for the maximum perturbation growth rate and its corresponding wave number. The breakup time is assumed to be the reciprocal of the maximum growth rate and the droplet radius is assumed equal to the wavelength of $\omega_{\max } . C_{3}$ is a model constant similar to $B_{1}$ in the $\mathrm{KH}$ model with typical values between 1 and 5.33 [62].

$$
\omega_{\max }=\sqrt{\frac{2}{3 \sqrt{3 \sigma}} \frac{\left[a\left(\rho_{l}-\rho_{g}\right)\right]^{\frac{3}{2}}}{\rho_{l}+\rho_{g}}}
$$




$$
\begin{gathered}
k_{\text {max }}=C_{3} 2 \pi \sqrt{\frac{3 \sigma}{a\left(\rho_{l}-\rho_{g}\right)}} \\
t_{b u}=\frac{1}{\omega_{\max }} \\
r_{\text {child }}=\frac{2 \pi}{k_{\max }}
\end{gathered}
$$

\subsection{Eddy Dissipation Concept}

The combustion model required for this work must have the ability to a) transiently model finite rate chemistry of a scheme consisting of several reactions and species, and b) account for the influence of turbulence on the reactions. With these requirements the Eddy Dissipation Concept (EDC) model was chosen [15]. The EDC is capable of solving infinitely fast chemistry, systems with fast and slow reactions, tabular and full chemistry, all with coupled turbulence [16]. In the EDC model the reaction terms are computed and combined with turbulent length and time scale terms to produce the final source term used in the CFD simulation. It is assumed that reactions occur in the fine scales of the turbulent field, whose length $\zeta^{*}$ is computed via Equation 62 over a time scale $\tau^{*}$ computed with Equation 63. Here $C_{\zeta}$ is the volume fraction constant equal to $2.1377, C_{\tau}$ is the time scale constant equal to 0.4082 , and $v$ is the kinematic viscosity.

$$
\begin{aligned}
\zeta^{*} & =C_{\zeta}\left(\frac{v \epsilon}{k^{2}}\right)^{1 / 4} \\
\tau^{*} & =C_{\tau}\left(\frac{v}{\epsilon}\right)^{1 / 2}
\end{aligned}
$$


$Y_{k}^{*}$ is the fine-scale mass fraction of species $k$, whose calculation is covered in the next sections, and $Y_{k}$ is the initial mass fraction of that species. Then $\dot{\omega}_{k}$ is input into Equation 4 and used in the CFD. Equation 64 thus describes the change in mass fraction of species $k$ that occurs over time $\tau^{*}$ and length scale $\zeta^{*}$, by processes described in sections 3.4.1 and 3.4.2.

$$
\dot{\omega}_{k}=\frac{\rho}{\tau^{*}\left[1-\left(\zeta^{*}\right)^{3}\right]}\left(Y_{k}^{*}-Y_{k}\right)
$$

\subsubsection{Shell Autoignition Model}

For this work it is desired that an ignition model be developed to simulate ignition of $\mathrm{C}_{10.17} \mathrm{H}_{19.91}$ fuel at a useful range of temperatures, pressures, and equivalence ratios. $\mathrm{C}_{10.17} \mathrm{H}_{19.91}$ though, like many hydrocarbon fuels, exhibits conditional ignition behaviour, namely two-stage behaviour at the lower range of a typical engine's operating conditions. Two-stage ignition is a phenomena exhibited by most hydrocarbon fuels where at relatively low temperatures $(600-950 \mathrm{~K})$ a radical pool is formed large enough so that branching and propagation reactions can occur. Branching processes are out-competed by propagation processes leading to the occurrence of a low-temperature cool flame [69]. If conditions allow, branching processes gain influence and a general ignition then occurs with a sharp rise in pressure. The first stage of the ignition process is a time where the fuel is being oxidized and radicals are being produced, but not in high enough concentrations to induce an abrupt rise in temperature. The second stage is the aforementioned cool flame which can eventually lead to a standard compression ignition event. Because this type of ignition is strongly a function of competing reactions, it cannot be described by single-step kinetics and a more advanced chemical model must be used. Figure 12 shows an 
experiment with a clear two-stage ignition event, where $\tau$ is the total ignition time, defined with respect to the time where piston compression ends $P_{C}$. Typically, compression ignition experiments are conducted in a Rapid Compression Machine (RCM) which is essentially a piston that stops once it reaches Top-Dead-Centre (TDC).

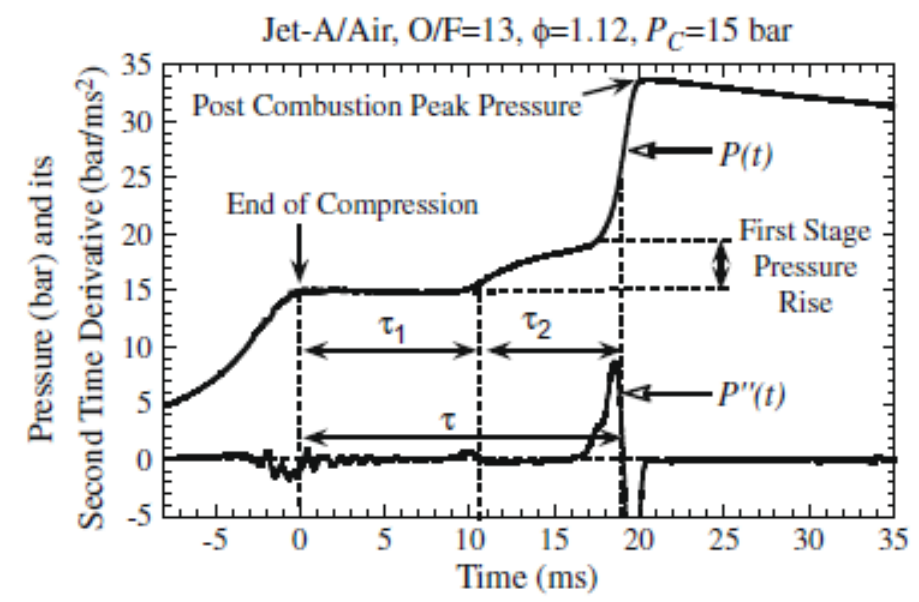

Figure 12 - Characteristic two-stage ignition event [70].

Equation 65 shows the additive description of ignition delay. $\tau_{1}$ begins at the end of compression and ends when the first pressure rise occurs. $\tau_{2}$ begins when the first pressure rise occurs and ends when the second pressure rise occurs.

$$
\tau=\tau_{1}+\tau_{2}
$$

To model the ignition of $\mathrm{C}_{10.17} \mathrm{H}_{19.91}$, the well-known Shell model was used, developed by Halstead in 1977 [71-72]. The Shell model is an 8-step reaction model with generic species capable of predicting the two-stage ignition behaviour of hydrocarbon fuels at low temperatures. The generic species represent groups of like intermediates in a degenerate branching process, those being: radical species $R$, propagating species $Q$, and 
branching species B. The Shell model has seen several modifications since 1977 and has been primarily used to simulate autoignition in gasoline and diesel engines, or isolated diesel spray combustion [73-83]. The Arrhenius constants for the general reaction mechanism must be tuned with experiments for each fuel used. Model constants have been reported for PRF RON 70, 90 and 100, TRF RON 89.5 and 99.6, 2-Me-hex-2-ene, n-decane, n-heptane, and tetradecane [72,81-82]. In this work the Shell model is being characterized for $\mathrm{C}_{10.17} \mathrm{H}_{19.91}$. The form of the model is based on the Schäpertöns/Lee approach with an additional species $\mathrm{T}$, which represents species that have been terminated from the degenerate branching process [80]. Table 1 shows the general Shell model mechanism. A species equation was solved for each species with the Eddy Dissipation Concept (EDC) model and above a certain temperature threshold the Shell model was deactivated and a single-step global mechanism was used.

Table 1. Original Shell model mechanism.

\begin{tabular}{|c|c|c|}
\hline Initiation & $\mathrm{RH}+\mathrm{O}_{2} \rightarrow 2 \mathrm{R}$ & $k_{q}$ \\
\hline \multirow{4}{*}{ Propagation } & $\mathrm{R} \rightarrow \mathrm{R}+\mathrm{P}$ & $k_{p}$ \\
\cline { 2 - 3 } & $\mathrm{R} \rightarrow \mathrm{R}+\mathrm{B}$ & $f_{1} k_{p}$ \\
\cline { 2 - 3 } & $\mathrm{R} \rightarrow \mathrm{R}+\mathrm{Q}$ & $f_{4} k_{p}$ \\
\cline { 2 - 3 } & $\mathrm{R}+\mathrm{Q} \rightarrow \mathrm{R}+\mathrm{B}$ & $f_{2} k_{p}$ \\
\hline \multirow{2}{*}{ Branching } & $\mathrm{B} \rightarrow 2 \mathrm{R}$ & $k_{b}$ \\
\hline \multirow{2}{*}{ Termination } & $\mathrm{R} \rightarrow \mathrm{T}$ & $f_{3} k_{p}$ \\
\hline & $2 \mathrm{R} \rightarrow \mathrm{T}$ & $k_{t}$ \\
\hline
\end{tabular}


The Schäpertöns/Lee modification essentially combines the first three propagation equations in order to preserve a mass balance in each equation. With this change the mechanism is transformed from that in Table 1 to what is shown in Table 2, where all species are conserved. This modification requires the following equation to form reaction products.

$$
R+(\Lambda+1)\left(\frac{1}{m} C_{n} H_{2 m}+p O_{2}\right) \rightarrow q P+f_{1} B+f_{4} Q+R
$$

Table 2. Schäpertöns/Lee mechanism.

\begin{tabular}{|c|c|c|}
\hline Initiation & $\mathrm{RH}+\mathrm{O}_{2} \rightarrow 2 \mathrm{R}$ & $k_{q}$ \\
\hline \multirow{3}{*}{ Propagation } & $\mathrm{R}+(\Lambda+1)\left(\frac{1}{\mathrm{~m}} \mathrm{C}_{\mathrm{n}} \mathrm{H}_{2 \mathrm{~m}}+\mathrm{pO}_{2}\right)$ & $k_{p}$ \\
& $\rightarrow \mathrm{qP}+\mathrm{f}_{1} \mathrm{~B}+\mathrm{f}_{4} \mathrm{Q}+\mathrm{R}$ & $f_{2} k_{p}$ \\
\cline { 2 - 3 } & $\mathrm{R}+\mathrm{Q} \rightarrow \mathrm{R}+\mathrm{B}$ & $k_{b}$ \\
\hline Branching & $\mathrm{B} \rightarrow 2 \mathrm{R}$ & $f_{3} k_{p}$ \\
\hline \multirow{2}{*}{ Termination } & $\mathrm{R} \rightarrow \mathrm{T}$ & $k_{t}$ \\
\cline { 2 - 3 } & $2 \mathrm{R} \rightarrow \mathrm{T}$ & \\
\hline
\end{tabular}

Several supporting equations are required to determine the molecular weights of the representative species and the ratio of products produced. Also, all rate equations follow Arrhenius kinetics other than $k_{p}$, which governs product formation. Given that all other reactions are assumed to be adiabatic and only the main products $\left(\mathrm{CO}_{2}, \mathrm{H}_{2} \mathrm{O}\right.$ and $\mathrm{CO}$, shown as $\mathrm{P}$ in Equation 66) have a non-zero enthalpy of formation, $k_{p}$ plays a very important role in determining heat release. The rate terms take the following forms where 
$A_{p n}, A_{n}, A_{f n}, E_{p n}, E_{n}, E_{f n}, x_{n}$ and $y_{n}$ are all unknown constants that need to be determined for each fuel.

$$
\begin{gathered}
k_{p}=\left[\frac{1}{k_{p 1}\left[O_{2}\right]}+\frac{1}{k_{p 2}}+\frac{1}{k_{p 3}[R H]}\right]^{-1} \\
k_{p n}=A_{p n} \exp \left(\frac{E_{p n}}{R T}\right), n=1,2,3 \\
k_{n}=A_{n} \exp \left(\frac{E_{n}}{R T}\right), n=q, b, t \\
f_{n}=A_{f n} \exp \left(\frac{E_{f n}}{R T}\right)\left[O_{2}\right]^{x_{n}}[R H]^{y_{n}, n=1,2,3,4}
\end{gathered}
$$

Since the representative species are not real species themselves, they must have molecular weights determined as a function of the specific fuel being used so that mass is conserved.

$$
\begin{gathered}
M W_{R}=\frac{M W_{C_{n} H_{2 m}}+M W_{O_{2}}}{2} \\
M W_{B}=2 M W_{R} \\
M W_{Q}=M W_{B} \\
M W_{T}=M W_{R}
\end{gathered}
$$

With the above parameters established, $\Lambda$ can be specified to satisfy Equation 66 . Further from Equation 66, $q$ and $p$ can be determined from the fuel, as in Equation 76, and from $\chi$ which is related to the ratio of carbon-based main products as shown in Equation 
77. The value of $\chi$ is a choice and is typically taken to be 0.67 as is the case with this work $[58,71,76-79,83]$.

$$
\begin{gathered}
\Lambda=\frac{f_{1} M W_{B}+f_{4} M W_{Q}}{M W_{C_{n} H_{2 m}} / m+p M W_{O_{2}}} \\
q=1+\frac{n}{m} \\
\frac{\left[C O_{2}\right]}{[C O]}=\frac{1-\chi}{\chi} \\
p=\frac{n(2-\chi)+m}{2 m} \\
P=\frac{\left[(n / m)\left(\chi C O+(1-\chi) C O_{2}\right)+H_{2} O\right]}{q}
\end{gathered}
$$

With the above system defined, the chemistry source terms can be defined with the following equation, where [i] is the molar concentration of species $i, v_{i}^{\prime \prime}$ and $v_{i}^{\prime}$ are the stoichiometric coefficients of the products and reactants, respectively, and $k_{j}$ is the rate term for reaction $j$. The specific form of each rate equation is given in Appendix A.

$$
\frac{d[i]}{d t}=\sum\left\{\left(v_{i}^{\prime \prime}-v_{i}^{\prime}\right) k_{j}\left(\prod[i]^{v_{i}^{\prime}}\right)\right\}
$$

\subsubsection{High Temperature Model}

The shell model has been proven to be able to predict low temperature (600-950K) kinetics of hydrocarbon fuels but it is not applicable to high temperature combustion. It is thus common practice to switch mechanisms when ignition is achieved and a high temperature is reached in a cell. For time-economy and practicality reasons, a single 
reaction model was chosen to represent the high temperature reaction. This reaction is simply the global mechanism for the oxidation of $\mathrm{C}_{10.17} \mathrm{H}_{19.91}$ and it switched on whenever the temperature in a cell exceeds $1100 \mathrm{~K}$. Table 3 shows the single-step mechanism and the reaction term which is determined with a standard Arrhenius equation. The model is coupled to the CFD (Equation 4) with Equations 80 and 62-64 as with the Shell Model.

Table 3. High temperature mechanism.

\begin{tabular}{|c|c|c|}
\hline Global & $\mathrm{C}_{10.17} \mathrm{H}_{19.91}+15.14 \mathrm{O}_{2}+56.95 \mathrm{~N}_{2}$ & $k_{h}$ \\
Reaction & $\rightarrow 10.17 \mathrm{CO}_{2}+9.95 \mathrm{H}_{2} \mathrm{O}+56.95 \mathrm{~N}_{2}$ & \\
\hline
\end{tabular}




\subsection{Validation Studies}

\subsection{Spray Model}

The formulation of the spray model used was the Euler-Lagrange Discrete Phase Model (DPM), which models individual parcels representing groups of droplets of similar character. The phases are modeled in a two-way coupled manner though no additional stochastic methods are used to model the inter-phase turbulence coupling. Droplet injection is done via the blob method where primary atomization is ignored and further breakup of the particles is handled solely with secondary breakup models. The KelvinHelmoltz (KH) and Rayleigh-Taylor (RT) models were used in a competing manner for breakup, where the KH model is used in the liquid core (liquid core here refers to a region with a high number density of droplets, not a liquid column) and the models compete outside of the liquid core. Avoidance of the primary breakup is known to be a very nonphysical assumption. To account for this possible source of error the constant $B_{1}$ from Equation 55 can be tuned to match experimental data [84-85]. It is also well known that gaseous phase turbulence has a large influence on fuel-air mixing For this reason several turbulence models were tested (for a detailed description of each spray model see Section 3.3).

The case being replicated is an experiment conducted by Sandia Laboratories of an n-Heptane spray. The nozzle being used has a single hole and is spraying pre-heated fuel (373 K) into a hot environment $(1000 \mathrm{~K})$ in order to simulate internal combustion enginelike conditions. Further details can be obtained directly from Sandia [86]. Liquid and vapour penetration results were obtained through time-averaged Mie-scattering and 
Schlieren visualization. The rail pressure was $154.33 \mathrm{MPa}$ and the ambient pressure was 1.33 MPa. An estimated $17.8 \mathrm{mg}$ of fuel was injected over a duration of $6.8 \mathrm{~ms}$. Simulations were conducted for the first $1.21 \mathrm{~ms}$. A schematic of the nozzle is shown in Figure 13.

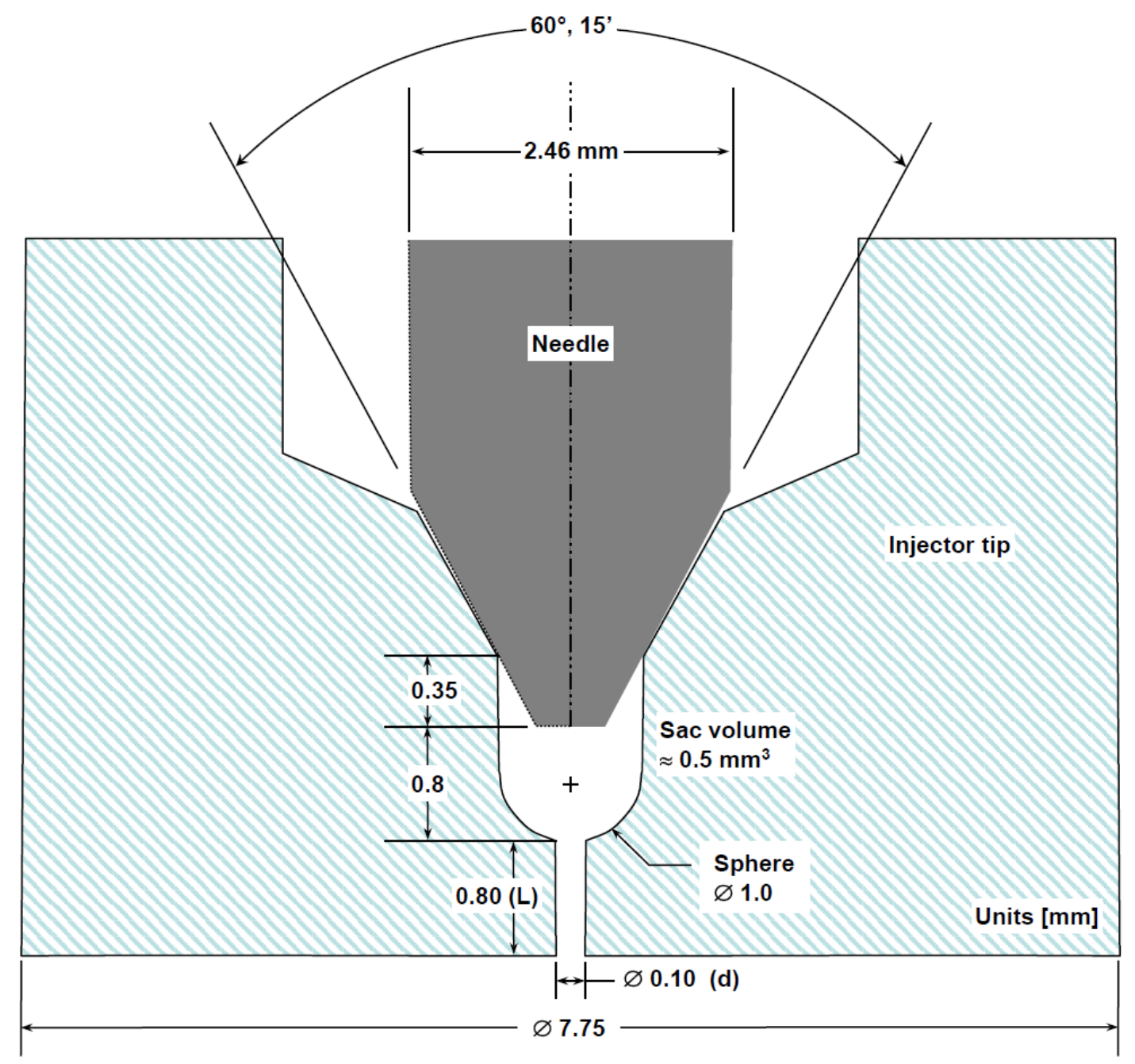

Figure 13 - Nozzle geometry [86].

The simulation geometry was chosen as a simple cylinder of a $2 \mathrm{~cm}$ diameter and $12.5 \mathrm{~cm}$ length. No periodicity was used to allow for the stochastic nature of the droplets as well as the non-uniformity of the LES simulations. In all cases it was verified that the size of 
the domain was large enough so that the far-field and exit boundary conditions did not affect the length or spread of the spray.

A weakness of the Euler-Lagrange multiphase model is that in many cases it is mesh dependent. The theory applied in the model assumes that the discrete phase occupies a small volume compared to that of the continuous phase (volume fraction of $<10-12 \%$ ). Numerically this statement must be true in every computational cell. In areas where the volume loading becomes high, the modeller has two options: selectively reduce the volume of discrete phase in given cells or enlarge the local mesh volume, thus sacrificing solution accuracy. Both cases result in a user-selected modification to the physics and neither option is fully satisfactory. For this reason, only a simple mesh independence study was conducted of two meshes, a original course mesh (M1) and a refined mesh (M2) constructed so that the volume loading would approach the limit of $10-12 \%$.

The ICEM-CFD software package was used to create each mesh, to ensure good mesh quality the 0-grid function was used on the inlet and outlet faces. Figure 14 shows an isometric view of the entire cylinder and Figures 15a and b show the face grids for M1 and M2. The number of nodes in the $z$ direction for M1 is 200 and 340 for M2. Table 4 lists some pertinent quality data for each mesh. Mesh quality is typically ranged from $0-1$, here, 1 represents a perfectly oriented cell while 0 represents the opposite. Poor mesh quality $(0 \sim 0.3)$ is known to adversely affect solution quality. In Fluent a minimum quality of 0.01 is recommended while a higher quality is stressed [21]. Skewness is ranged from $0-1$ and is a measure of a cell's deviation from its ideal equilateral shape. Here 0 represents the ideal shape and 1 represents a sliver which is two coplanar nodes in 3D or 2 collinear nodes in 2D. In Fluent it is recommended that maximum skewness be below 0.95 to avoid 
convergence difficulties [21]. Aspect ratio is the ratio of a cells edges, here a value of 1 is desired (representing an equilateral cell) and any values above 1 represent non-equilateral cells. Table 4 lists the quality of the mesh elements with the lowest quality (minimum quality), the skew of the elements with the highest skew (maximum skewness) and the aspect ratios of the elements with the highest aspect ratios (maximum aspect ratio), all of which are well within acceptable limits.

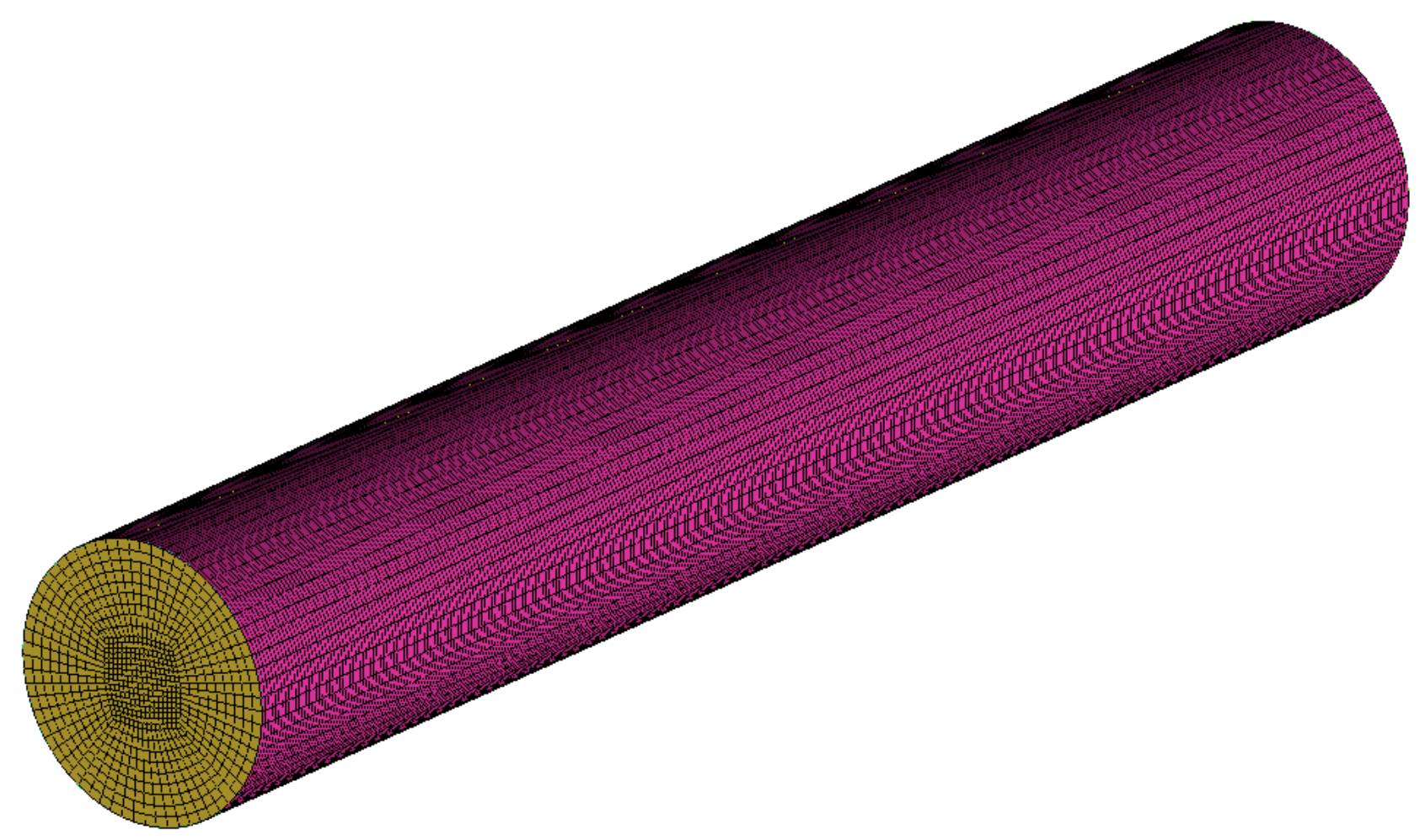

Figure 14 - Isometric view of entire domain. 

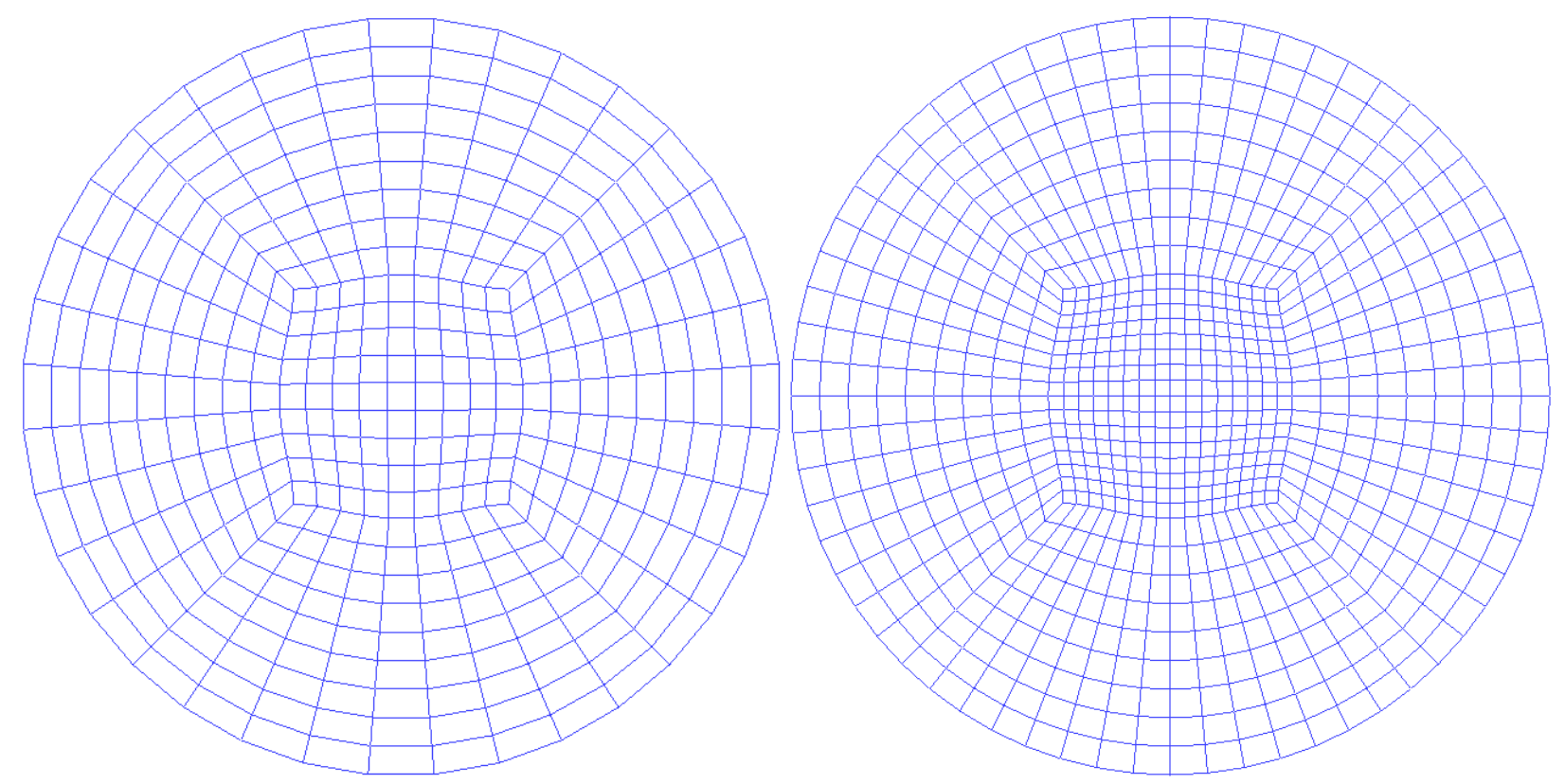

Figures 15a and $b$ - Face views for M1 and M2.

Table 4 - Mesh Data

\begin{tabular}{|c|c|c|c|c|}
\hline Mesh & Minimum Quality & $\begin{array}{c}\text { Maximum } \\
\text { Skewness }\end{array}$ & $\begin{array}{c}\text { Maximum Aspect } \\
\text { Ratio }\end{array}$ & Number of Nodes \\
\hline M1 & 0.6 & 0.65 & 2.67 & 84800 \\
\hline M2 & 0.65 & 0.6 & 2.57 & 294100 \\
\hline
\end{tabular}

All cases were simulated using a transient semi-implicit formulation and second order upwind discretization for all variables. The coupled solver was used which solves the momentum equations, a pressure corrected mass equation and the energy equation in a coupled manner while the turbulence and DPM equations are solved individually. A time step size of $1.51 \mathrm{e}-06$ was chosen so that the particles were calculated at least once per cell. Solution convergence was obtained for every equation at every time step at a residual level 
of 1e-06. The case has a pressure outlet downstream of the spray and a symmetry condition on the side walls. The physical inlet boundary in the case is a wall. No nozzle geometry is modeled. The injection point is located along the axis of the cylinder $1.25 \mathrm{~mm}$ from the inlet wall.

There are a significant amount of variables to test while spray modelling; a complete test of variables has not been completed in this work. Only the most influential variables were tested, those being: Eulerian turbulence model, mesh density and $\mathrm{KH}$ breakup constant $B_{1}$. Testing these three variables led to 44 simulations running between $4-10$ hours each. The number of injected parcels, initial variance of droplet sizes, time step dependence, RT breakup constant $C_{3}$ and level of free stream turbulence were not tested. Firstly each turbulence model was tested over a range of $B_{1}$ with M1 so that a suitable range of $B_{1}$ could be determined The best validated cases where then tested with M2 over the reduced $B_{1}$ range. The Spalart-Allmaras, Realizable k- $\varepsilon$, Reynolds Stress Method (RSM) and LES with the Smagorinsky-Lilly model were used and compared.

Simulations of a range of $B_{1}$ of 1-50 were conducted for each turbulence model although the appropriate range for this nozzle is deemed to be between 1 and 5 . Each simulation lasted $1.2 \mathrm{~ms}$, this time was chosen because it was relevant for the prechamber studies to be explained later. Figures 16 to 21 show the vapour and liquid penetration graphs for each turbulence model aside from the Spalart-Allmaras model, for which the results were unsatisfactory, Figures 22 and 23 compare the best cases with each turbulence model. The best results were obtained with the LES turbulence model with a $B_{1}$ of 1 when considering validation with both vapour and liquid lengths. The maximum errors 
for the LES, RSM, Realizable k- $\varepsilon$ and Spalart-Allmaras models were $10 \%, 24.3 \%, 30.5 \%$ and $68.8 \%$ respectively over the range tested. The behavioural differences in the vapour length curves between turbulence models are large when comparing the RANS models with LES. All turbulence models predicted the initial vapour length well, corresponding to the transient portion of the spray when the vapour length is determined by the liquid core. After a certain amount of time all the RANS models began to under-predict the vapour length significantly, this can be seen after $0.3 \mathrm{~ms}$ with the $\mathrm{k}-\varepsilon$ case and after $0.4 \mathrm{~ms}$ with the RSM case (See Figures 18 and 20). The $B_{1}$ variation had a negligible effect on the gaseous phase with the RANS models while Figure 16 shows that $B_{1}$ variations affected the LES solution. $B_{1}$ had a significant effect on the liquid length for all cases. A larger $B_{1}$ means that the characteristic break-up time is longer, thus liquid penetration is higher. The effect of varying $B_{1}$ for each turbulence model is different, however the appropriate range can be said to be within 1-1.73.

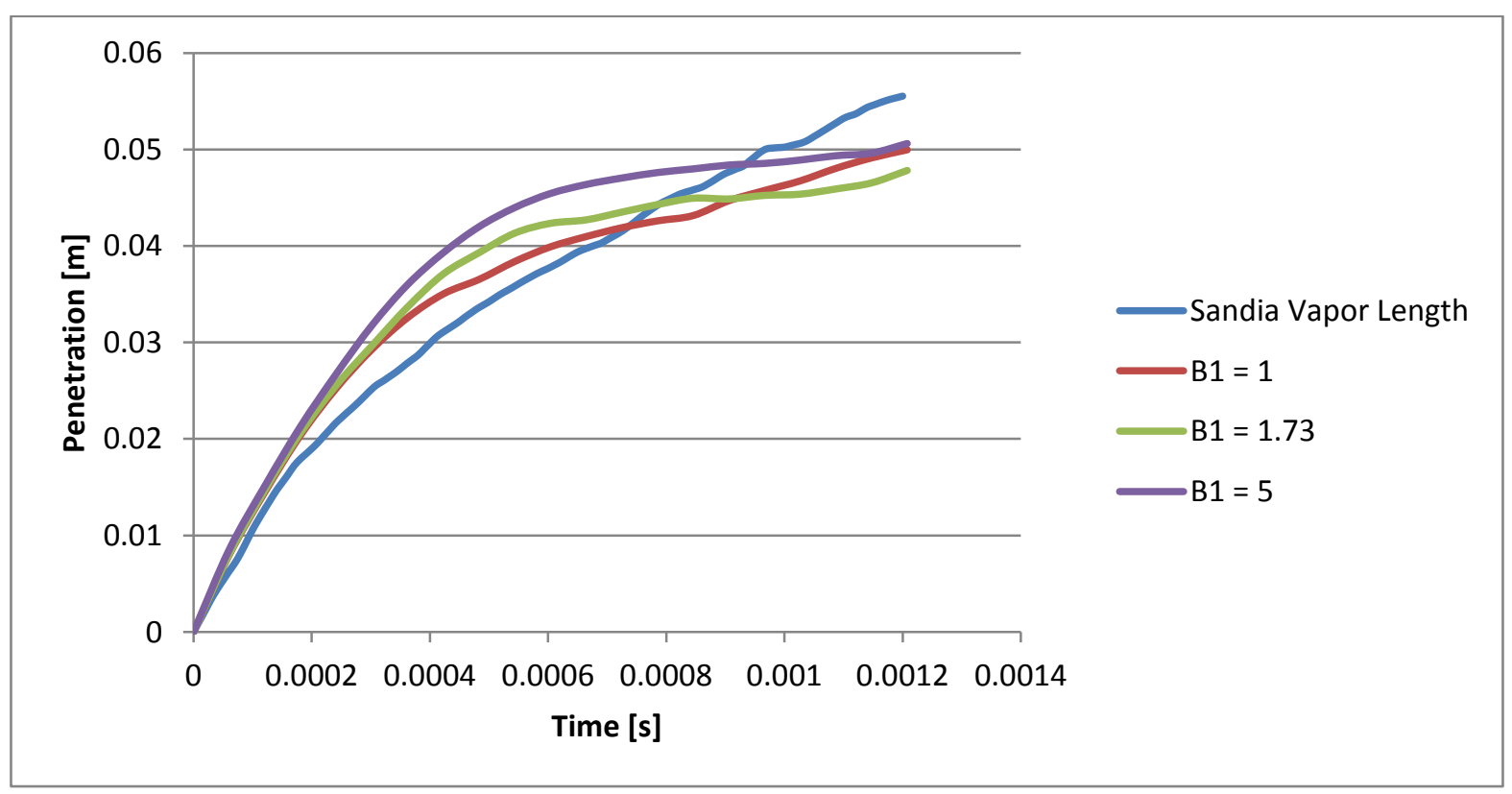

Figure 16 - Vapour length validation results for the LES model. 


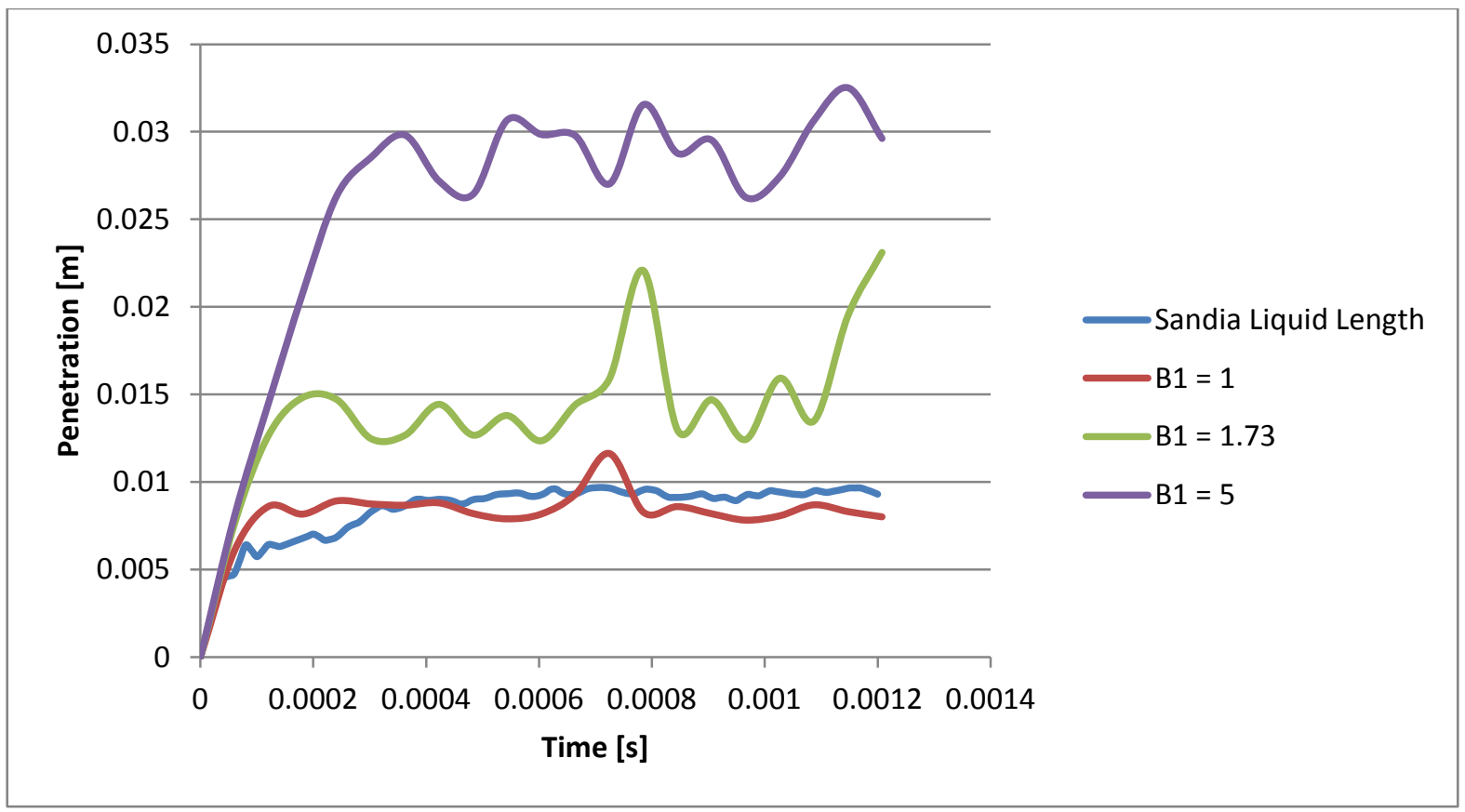

Figure 17 - Liquid length validation results for the LES model.

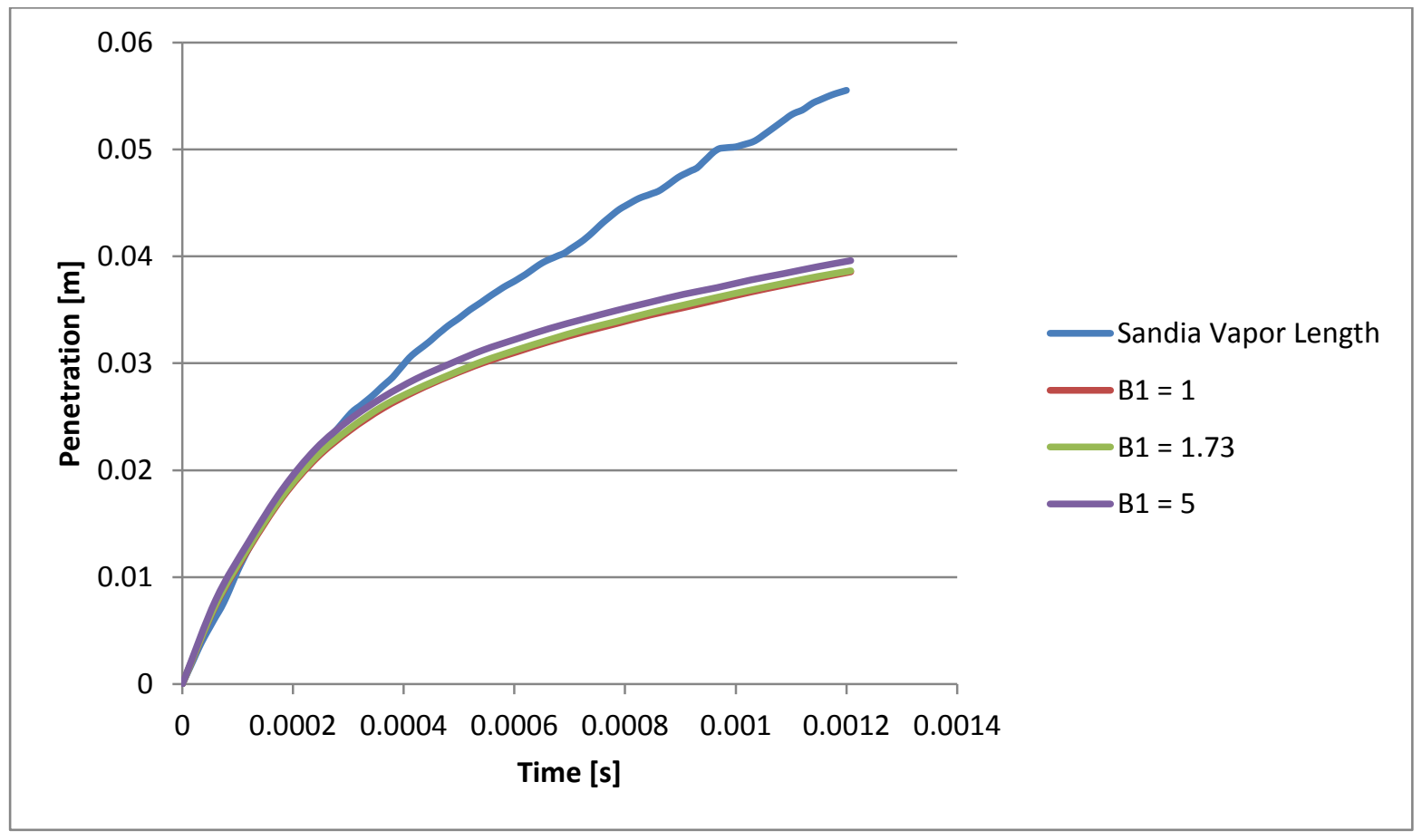

Figure 18 - Vapour length validation results for the Realizable $k-\varepsilon$ model. 


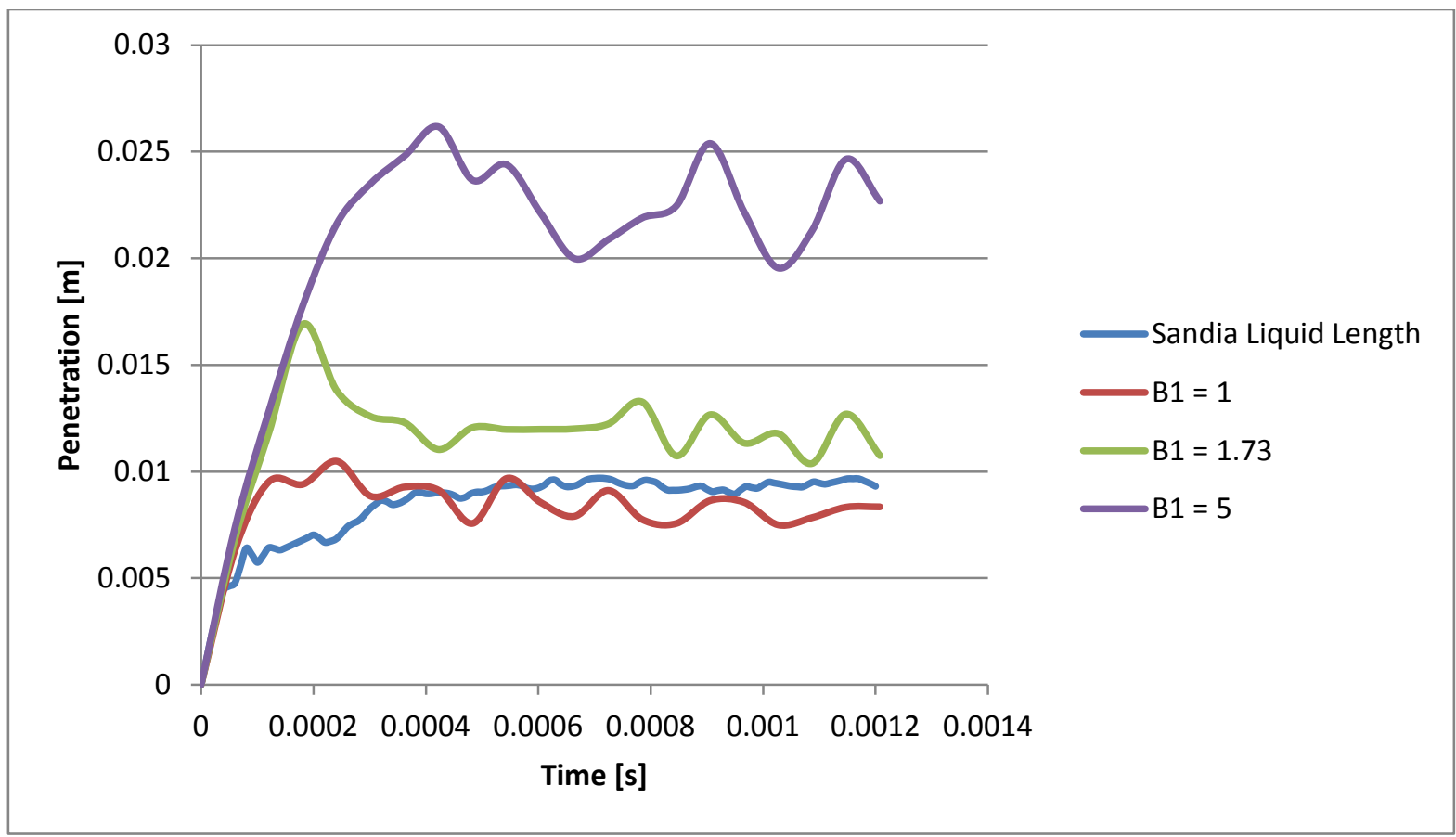

Figure 19 - Liquid length validation results for the Realizable $k-\varepsilon$ model.

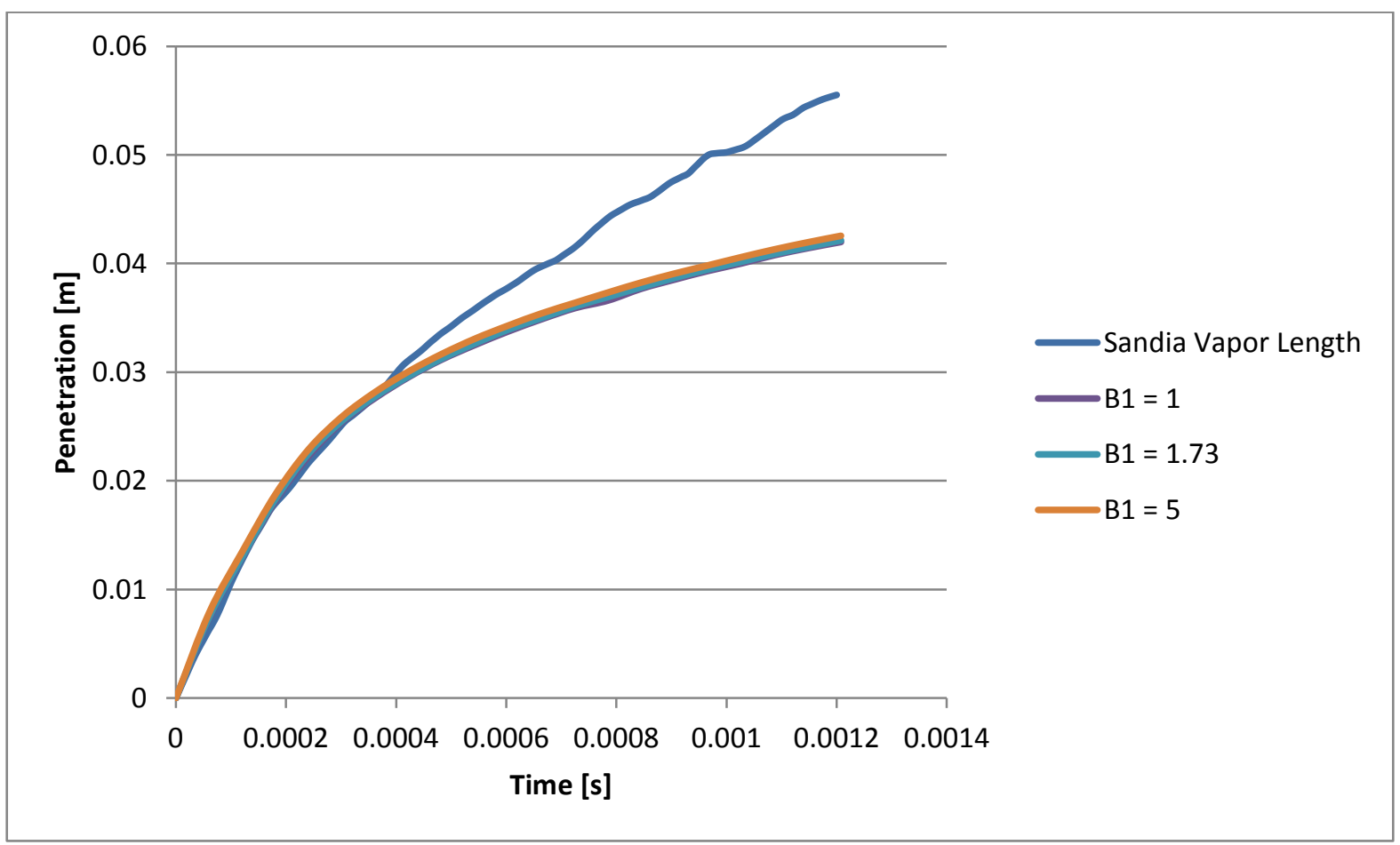

Figure 20 - Vapour length validation results for the Reynolds Stress Model. 


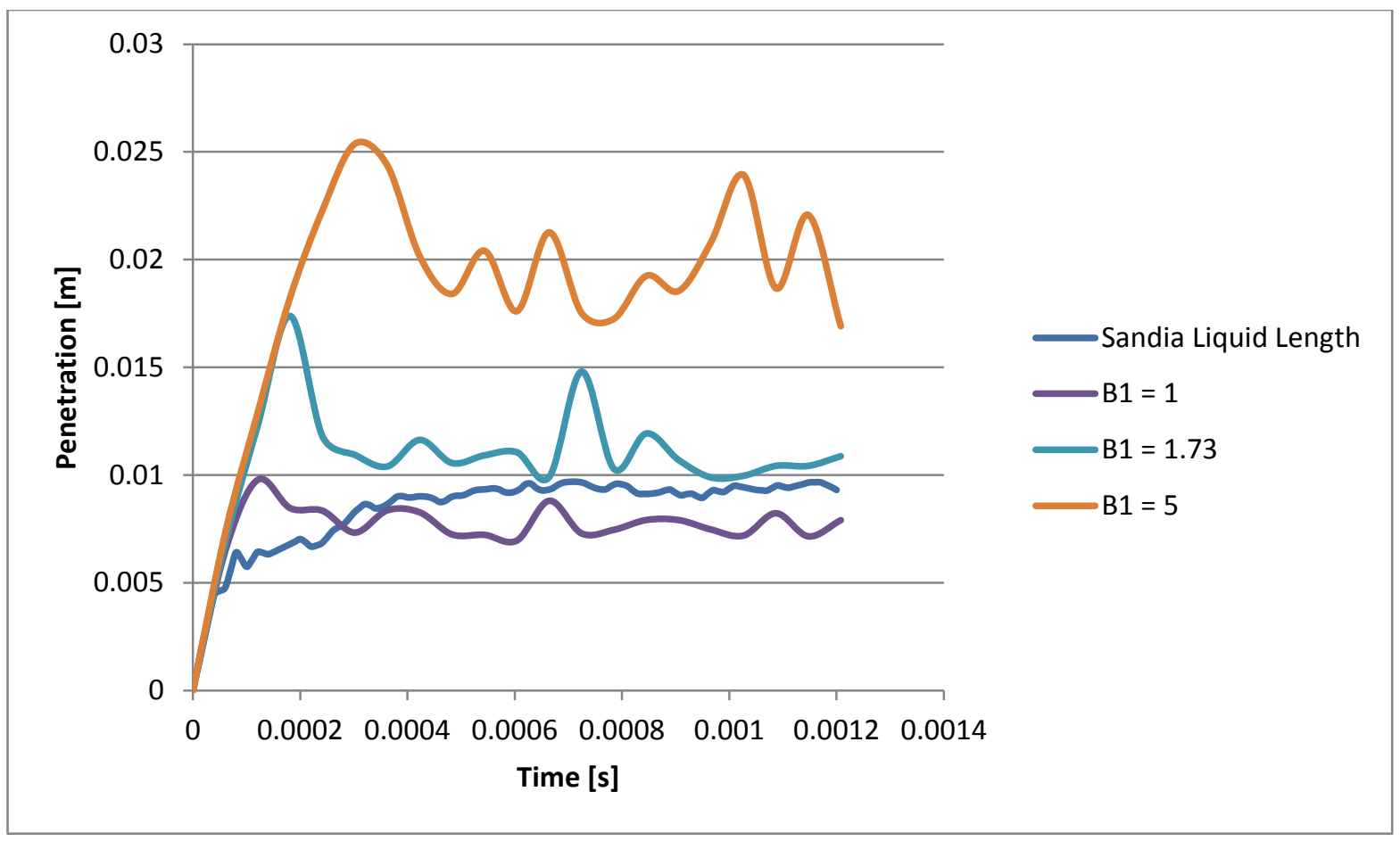

Figure 21 - Liquid length validation results for the Reynolds Stress Model.

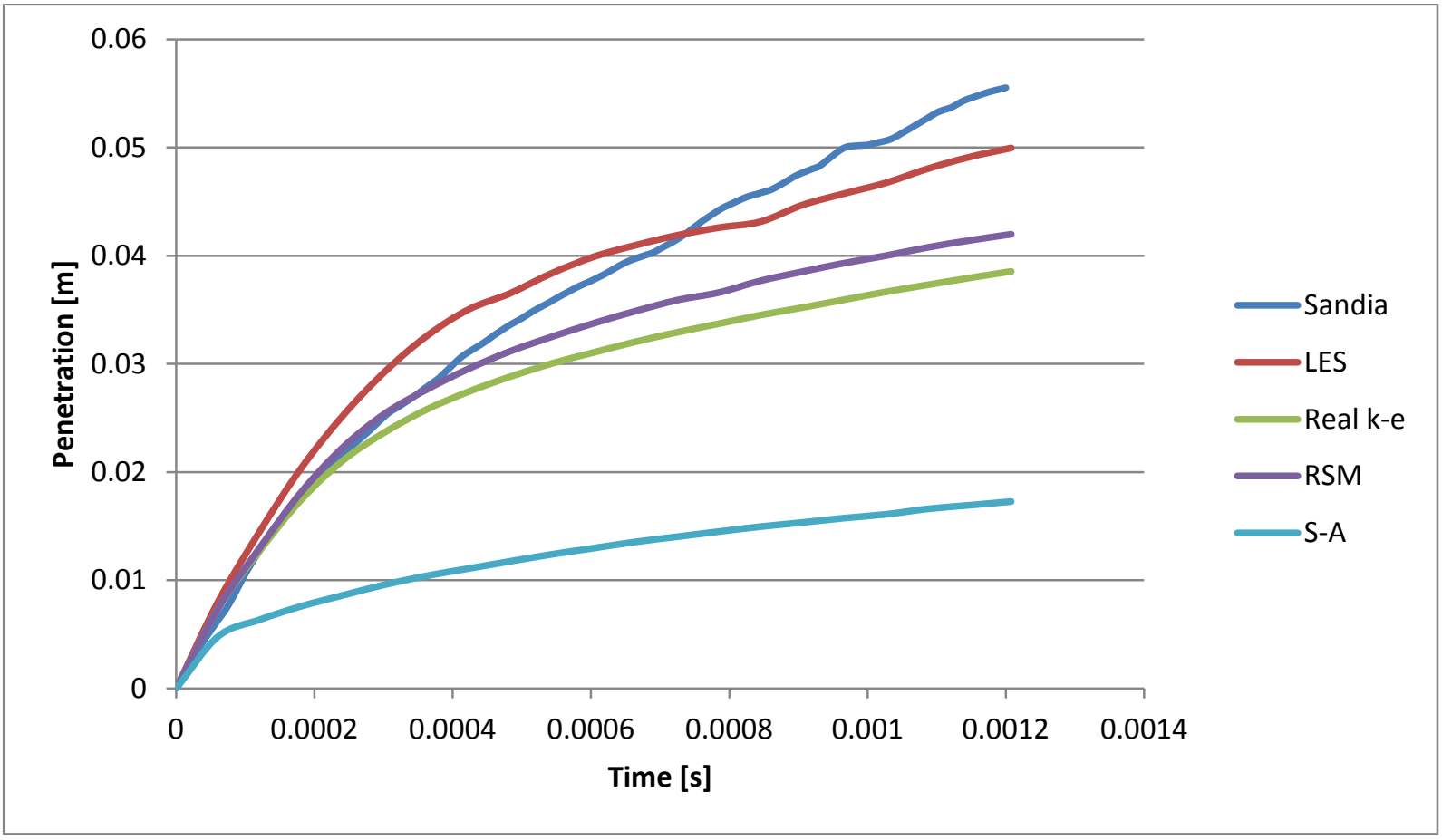

Figure 22 - Vapour length comparison with all turbulence models. 


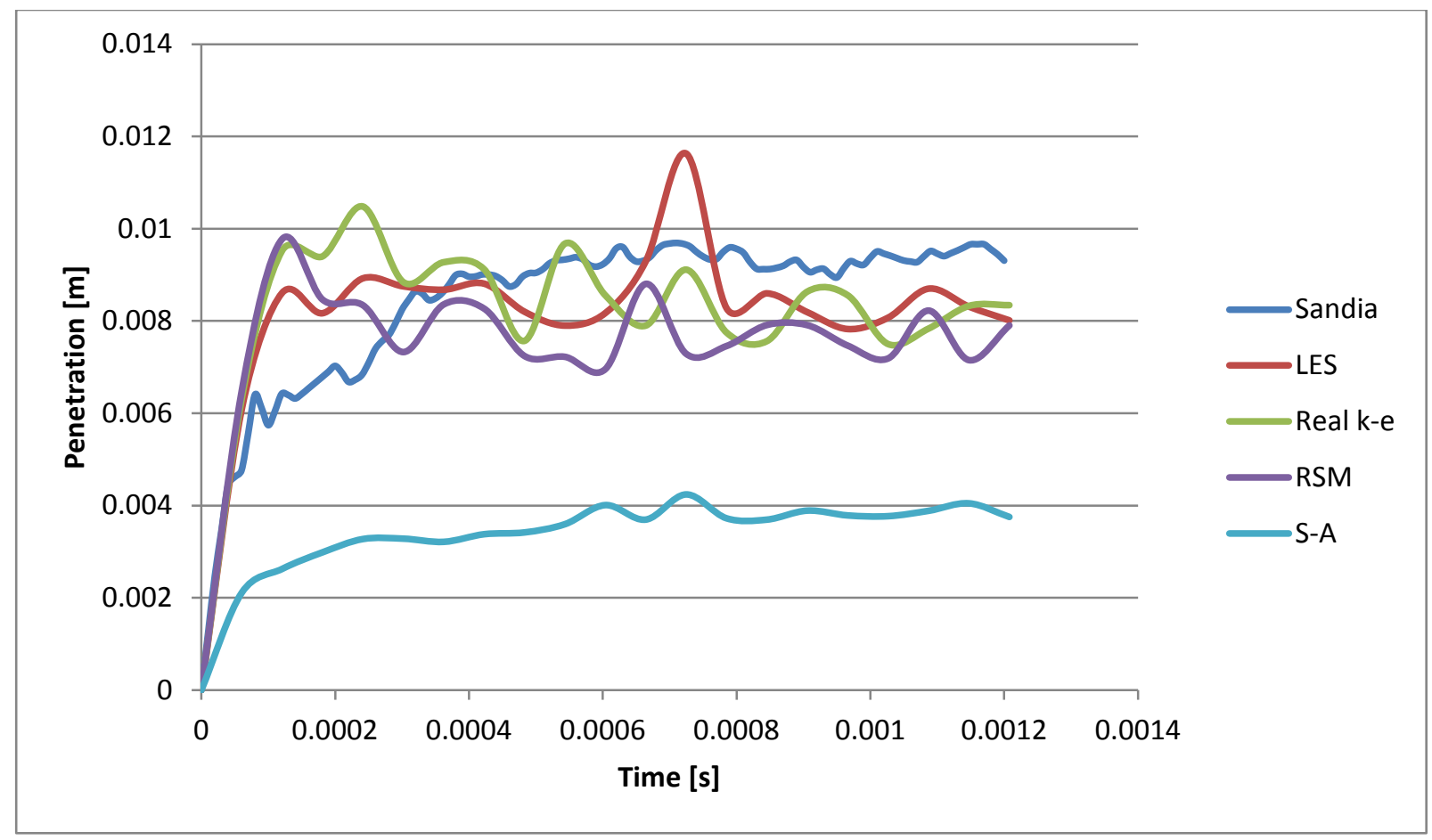

Figure 23 - Liquid length comparison with all turbulence models.

After tuning several variables and testing of different turbulence models for both the gas and liquid phase, a good match was determined with experimental data for predictions of both liquid and vapour length. The best case was that with the LES model with a $B_{1}$ of 1 . The most dominant modelling choice in the system was the gas phase turbulence model, as the results with the LES model differed greatly from that of the Realizable k- $\epsilon$, RSM and Spalart-Allmaras models. In most cases the variable $B_{1}$ had a small effect on the vapour penetration but a very large effect on the liquid penetration. For all cases it was most suitable to reduce the value of $B_{1}$ close to or at the minimum reported value of 1 . In future work it would be ideal to be able to validate results at different operating conditions, primarily with different rail pressures and different fuels. At this point, it is this author's opinion that the two weakest points in this type of spray modelling 
are the prediction of the injection velocity and the DPM mesh dependency. This injection velocity needs to be determined from detailed knowledge of the nozzle condition which represents a significant added complexity to the system. The reduced mesh sensitivity requires a largely new numerical treatment of the particles, both of which are formidable tasks. Thus, future work should be directed towards alleviating these issues. The model as is, is considered to be acceptable as the final configuration (shown in Figure 24) has a maximum error of $10 \%$ over the range tested.

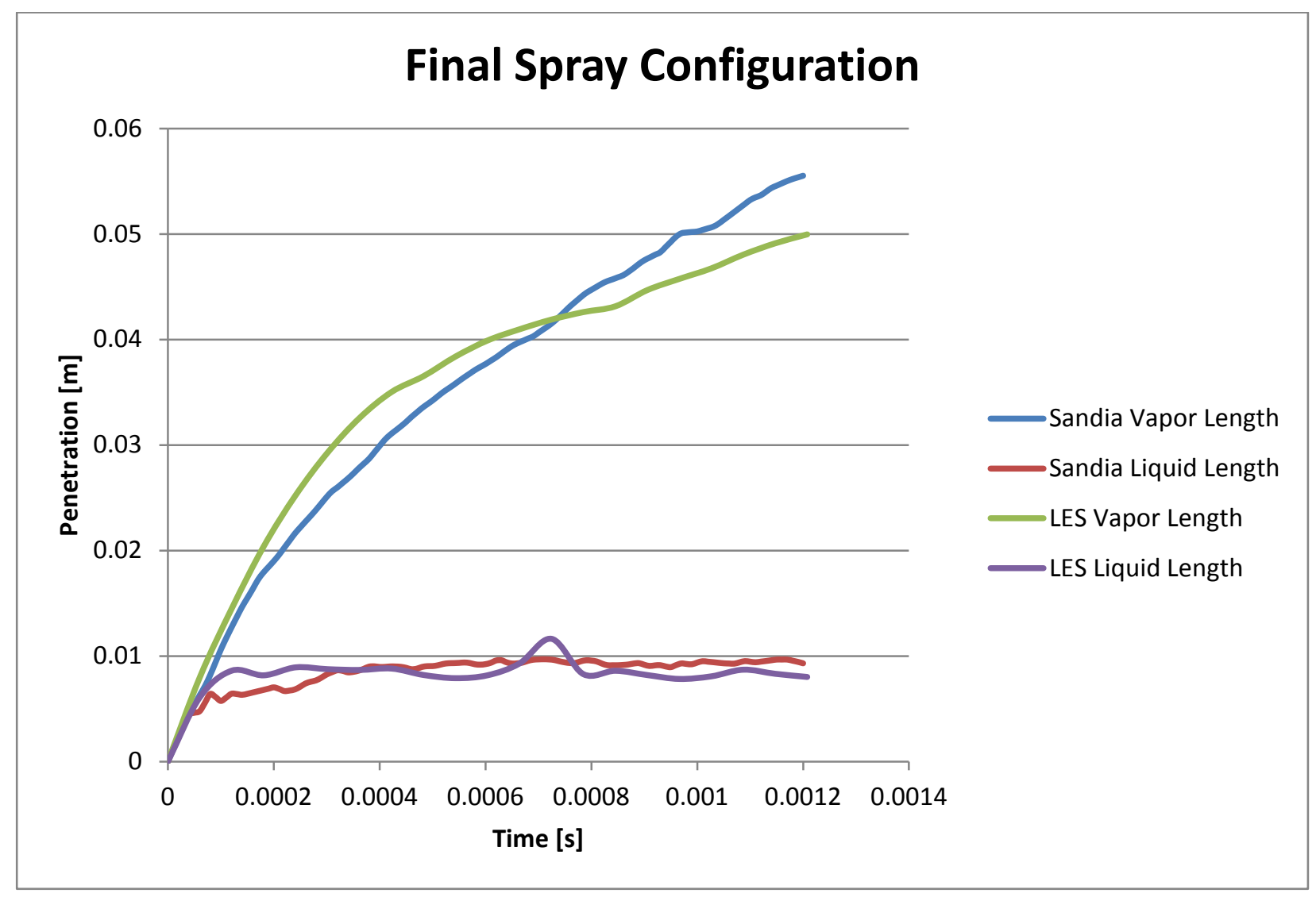

Figure 24 - Vapour and liquid length validation for final LES case. 


\subsection{Shell Model}

To validate the behaviour of the Shell model, Rapid Compression Machine (RCM) experiments were used with various $\mathrm{C}_{10.17} \mathrm{H}_{19.91}$ fuel/oxidizer mixtures at various thermodynamic conditions. An RCM is essentially a piston which has an initial gas mixture at a known temperature and pressure that moves only from Bottom-Dead-Centre (BDC) to Top-Dead-Centre (TDC) in order to raise the temperature and pressure of the mixture. Once at TDC no further motion of the piston is allowed and measurements of the gas phase can be taken without any effects due to volume change (such as the return to BDC in a piston engine). RCM experiments share a similar operation to piston engines and thus they make enabling devices for experimental studies of compression-ignition. All experiments are referenced by their end-of-compression temperature and pressures, being those of the state that exists at TDC, allowing a consistent reference for experiments that have different strokes and clearance volumes. The downside to this reference choice is that no flow field information regarding the events that led to this reference state are considered. For example, two different experiments with two different end-of-compression states will likely have different end-of-compression flow fields (velocity, turbulent fields etc.). By characterizing the experiments by only end-of-compression temperature and pressure, the variation in all other parameters of the flow fields between experiments are ignored. This characterization essentially implies the assumption that the primary state parameters affecting ignition delay time are temperature and pressure and not pre-existing velocity fields - an assumption that may be true in idealized experimental cases but not in cases with significant aerodynamic strain on the chemistry. Thus, these RCM experiments are only claimed to predict the chemical ignition delay and not the mechanical ignition delay. 
The effect of mechanical compression induced vortices on the chemistry of RCM devices has been studied with the general conclusion being that creviced pistons can somewhat mitigate "roll-up vortices". However, the pre-existing flow field at end-of-compression conditions still affects the chemistry [87-91]. For this reason it was chosen to model the compression process so that initial conditions can be defined at the beginning of compression and not the end when conditions are less likely to be homogeneous.

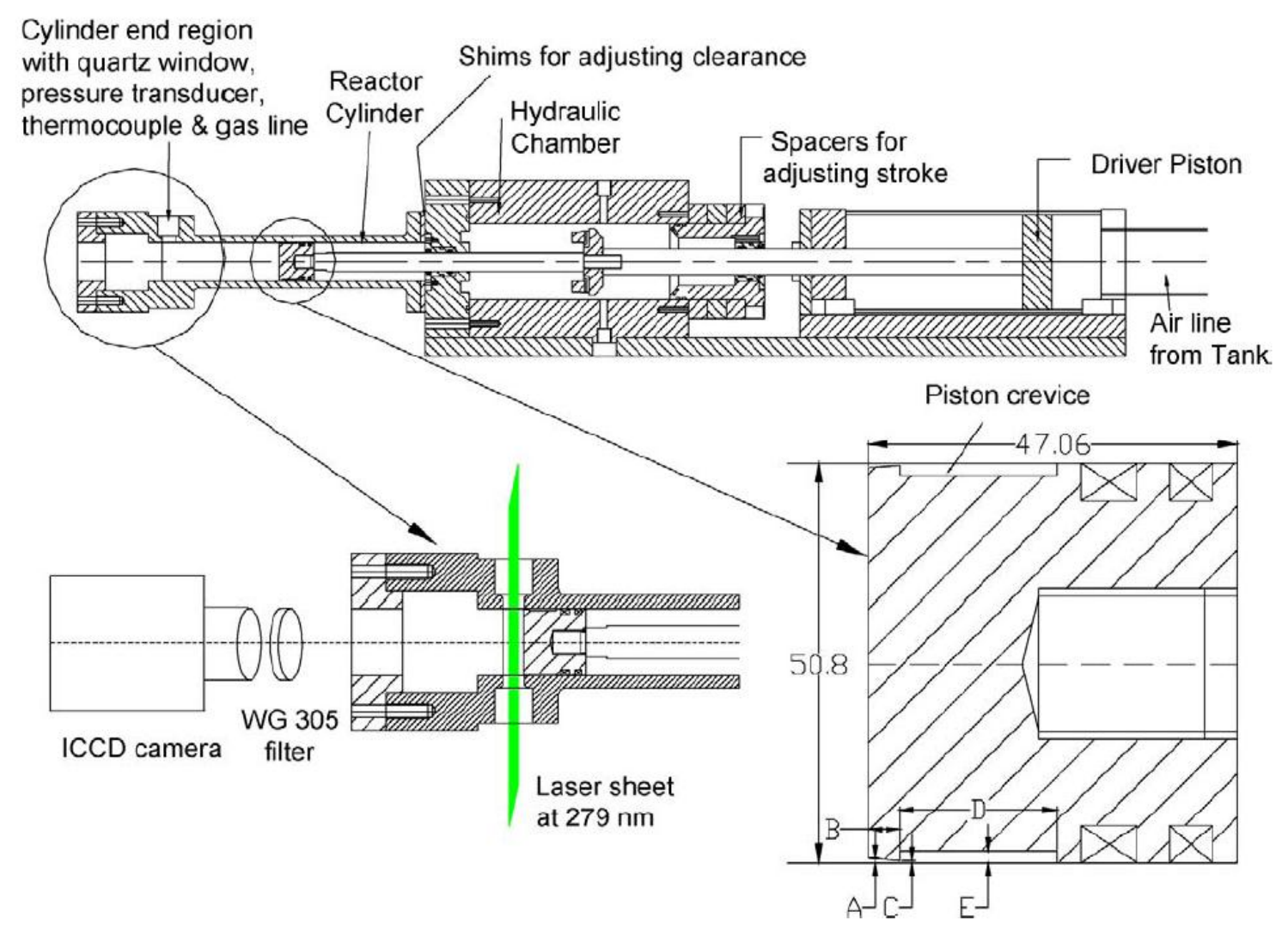

Figure 25 - RCM Schematic [87].

The RCM used by Mittal and Sung as shown in Figure 25 consists of driver and reactor cylinders of 5 and 2 in bores respectively, and stroke is variable between 7 and 10 
in. Compression strokes last approximately $30 \mathrm{~ms}$ with a stroke of $10 \mathrm{in}$. With a pressure transducer and thermocouple located in the cylinder, end pressure-time or temperaturetime angle data can be recorded and compared to numerical results. A variation in incylinder temperatures and pressures between experiments can be obtained by varying the stroke. The stroke can be adjusted by adding or removing spacers, while the clearance volume can be adjusted by using shims. Planar laser-induced fluorescence (PLIF) of an acetone tracer in nitrogen was used with an intensified CCD camera to characterize the temperature field during the experiment.

An in-depth study of the ignition of $\mathrm{C}_{10.17} \mathrm{H}_{19.91}$ (04POSF4658) and JP-8 was carried out using the previously described apparatus by Kumar and Sung in the end-ofcompression ranges of $650-1100 \mathrm{~K}$ and $7-30$ bar with an equivalence ratio range of 0.42 2.26 [70]. This test database was made available and served as the basis of validation for all results in this section.

The simulation geometry was chosen to be a $45^{\circ}$ cut of a simple cylinder of radius 1 in and length $7 \mathrm{in}$. The length of the cylinder was not changed in order to simulate a change in stroke in the RCM, instead the mesh was deformed with a different time history in order to attain the same end of compression temperature and pressure as in the specific experiment.

ICEM-CFD was again used to develop the mesh with the O-grid function, here all faces of the model are walls to simulate an enclosed vessel. The grid dimensions were $22 \times 30 \times 200$ in the $r, \theta$ and $z$ directions respectively. The grid was kept at a similar size as the grid used for the spray validation. Ideally, since this is a single-phase simulation it 
would be best to decrease the mesh size continually until a mesh independent solution is obtained. In this case however, the mesh size is limited by the two-phase nature of the spray and thus there is little purpose in validating one model on a much more dense mesh than another model when both models will end up coupled at a later stage. To ensure that mesh-dependence of the chemistry model was not severe, tests were ran with meshes of two and four times the density with a maximum change in results of $5 \%$. The decided-upon mesh is shown in Figure 26.

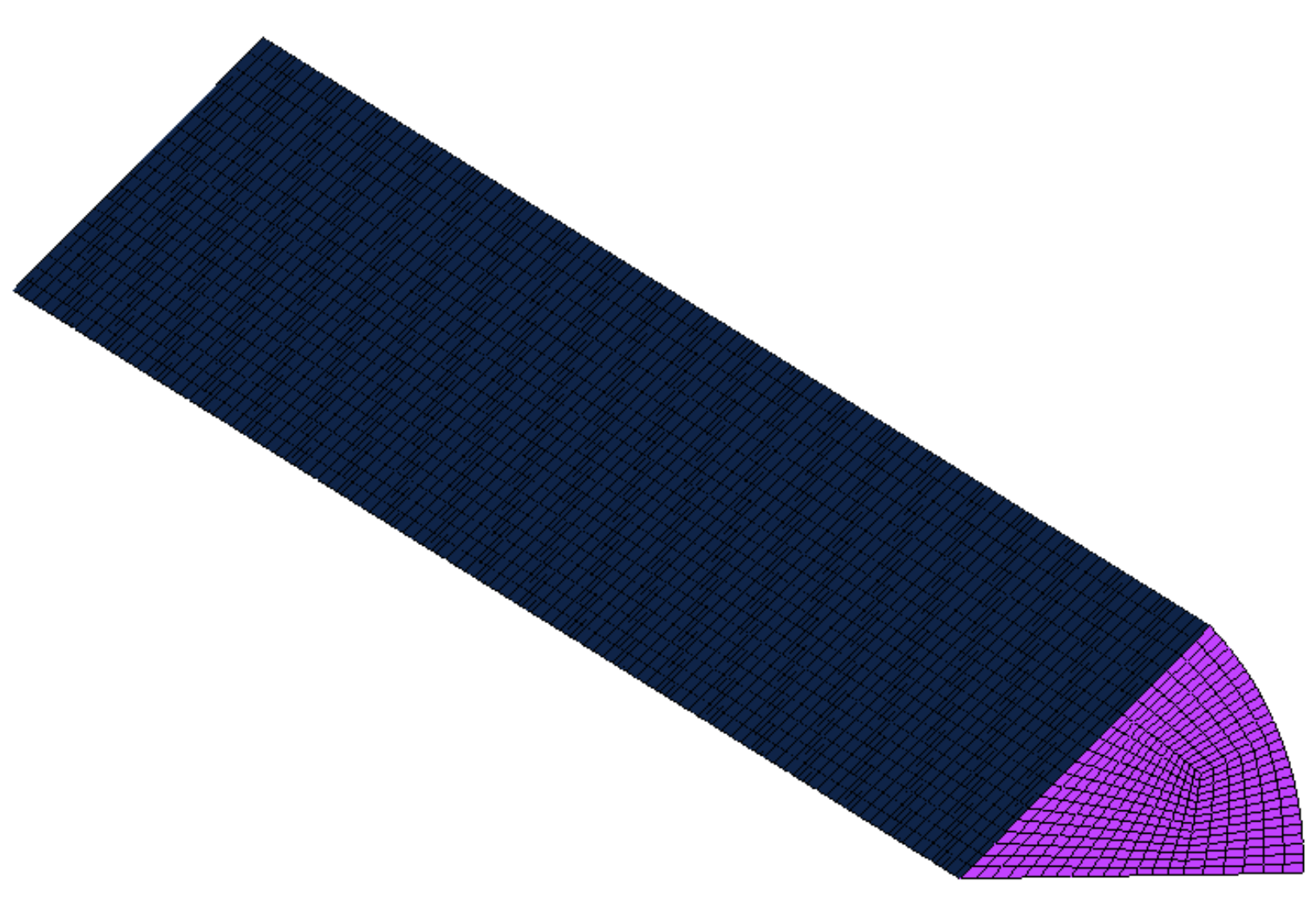

Figure 26 - Isometric view of RCM mesh.

To effectively model a piston, all dynamic meshing characteristics were handled with built-in functions in Fluent, those being: crank period, crank angle step size, stroke, and connecting rod length. The Eddy Dissipation Concept (EDC) model was used with a 
User Defined Function (UDF) to calculate the species source terms from the Shell model. The Realizable k- $\varepsilon$ model was used with a time step size of $8.3 \mathrm{e}-05 \mathrm{~s}$ or two time steps per crank angle. Simulations were also tested at four time steps per crank angle with negligible differences. The solver settings were set the same as with the spray validation, those being: a coupled semi-implicit solver with second order upwind discretization with the convergence criteria for all variables set at $1 \mathrm{e}-06$. The initial gas temperature and pressure were varied along with initial equivalence ratio in order to produce end-of-compression conditions that matched the experiments. Within the EDC model a volume fraction constant of 2.1377 and a time scale constant of 0.4083 were used. The Ordinary Differential Equation (ODE) error tolerance was set to $1 \mathrm{e}-14$ for both the absolute and relative tolerances because solution errors were found at the default values. The molecular weights were determined from Equations 71-74 while the $c_{p}$ and $\Delta h_{f}^{o}$ for the intermediate species: T, Q, B and R were set to zero to ignore any direct contribution of intermediate species production on heat release. All other properties of the intermediate species were set to that of nitrogen.

Four conditions were simulated and compared with experimental data at two different end-of-compression pressures and two different initial equivalence ratios. The conditions are summarized in Table 5. These equivalence ratios and pressures were chosen because they cover a range likely to occur in an engine and they display obvious two-stage ignition behaviour. Each condition was tested over an appropriate end-of-compression temperature range, typically from 650-900 K. 
Table 5 - RCM conditions simulated.

\begin{tabular}{|l|l|l|}
\hline Condition & Equivalence Ratio $\Phi$ & Pressure $P_{c}[\mathrm{bar}]$ \\
\hline 1 & 1.12 & 7 \\
\hline 2 & 1.12 & 15 \\
\hline 3 & 0.76 & 7 \\
\hline 4 & 0.76 & 15 \\
\hline
\end{tabular}

A typical temporal pressure curve is shown in Figure 27 comparing experimental results and simulation results from the Shell model. After the end of compression at $30 \mathrm{~ms}$ both curves show a short time with no pressure rise, this is the first ignition delay, then at roughly $50 \mathrm{~ms}$ both curves show the main ignition event, the time in between these events being the second ignition delay. It is immediately obvious that the Shell model is overpredicting pressure in the second ignition delay stage, this is a feature shared by all of the conditions tested in this work. Given that only the main products $\left(\mathrm{CO}_{2}, \mathrm{H}_{2} \mathrm{O}\right.$ and $\left.\mathrm{CO}\right)$ can directly raise the pressure it is clear that the reaction rates for these species are being overpredicted. As long as this higher pressure occurring in the second ignition delay region does not affect $\tau_{2}$ then it is taken to be of little consequence because the value of $\tau_{2}$ (the timing of the main ignition event) is much more important than the gas composition prior to the main ignition event. In reality, the main ignition event is a function of the prior gas composition which consists of a large number of radical species being created and consumed in multiple reactions, the Shell model however uses a reduced chemical mechanism where only one radial species and three intermediate species are simulated. 
Thus it is reasonable that the gas composition simulations would not match that of the experiment at every point in time.

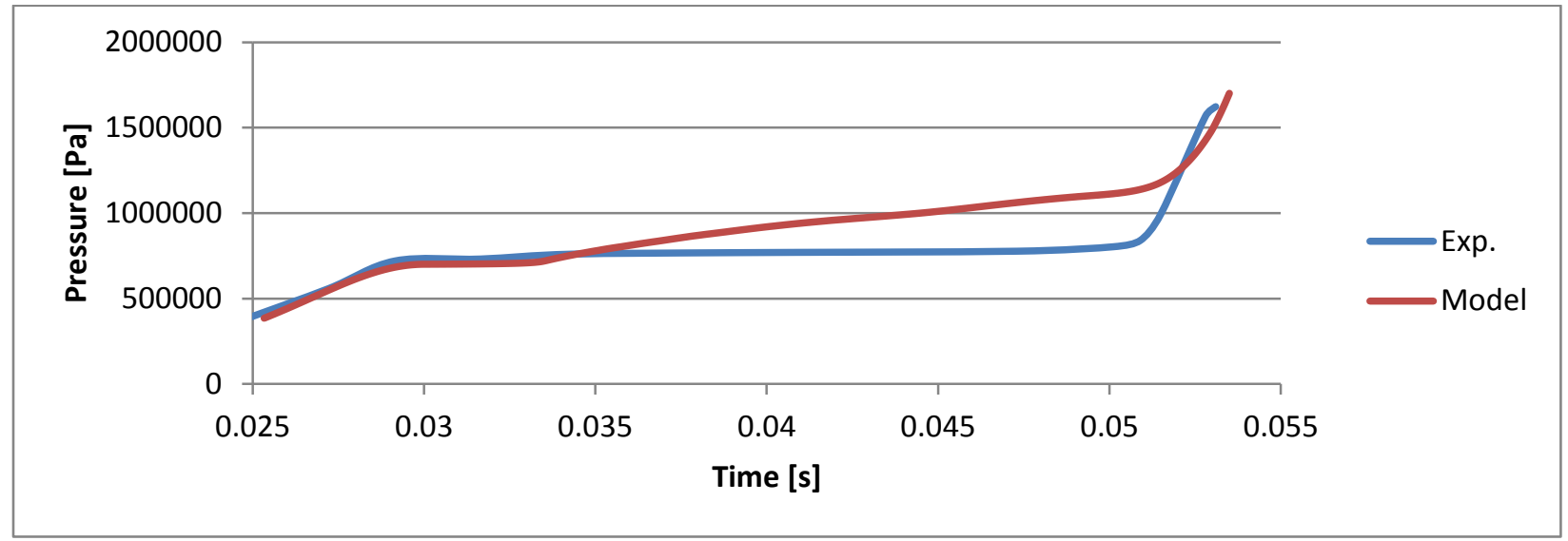

Figure 27 - Shell model comparison with RCM experiment.

Figures 28-31 show the results for total ignition delay $\tau$ as a function of end-ofcompression temperature $T_{C}$ for the four conditions previously mentioned.

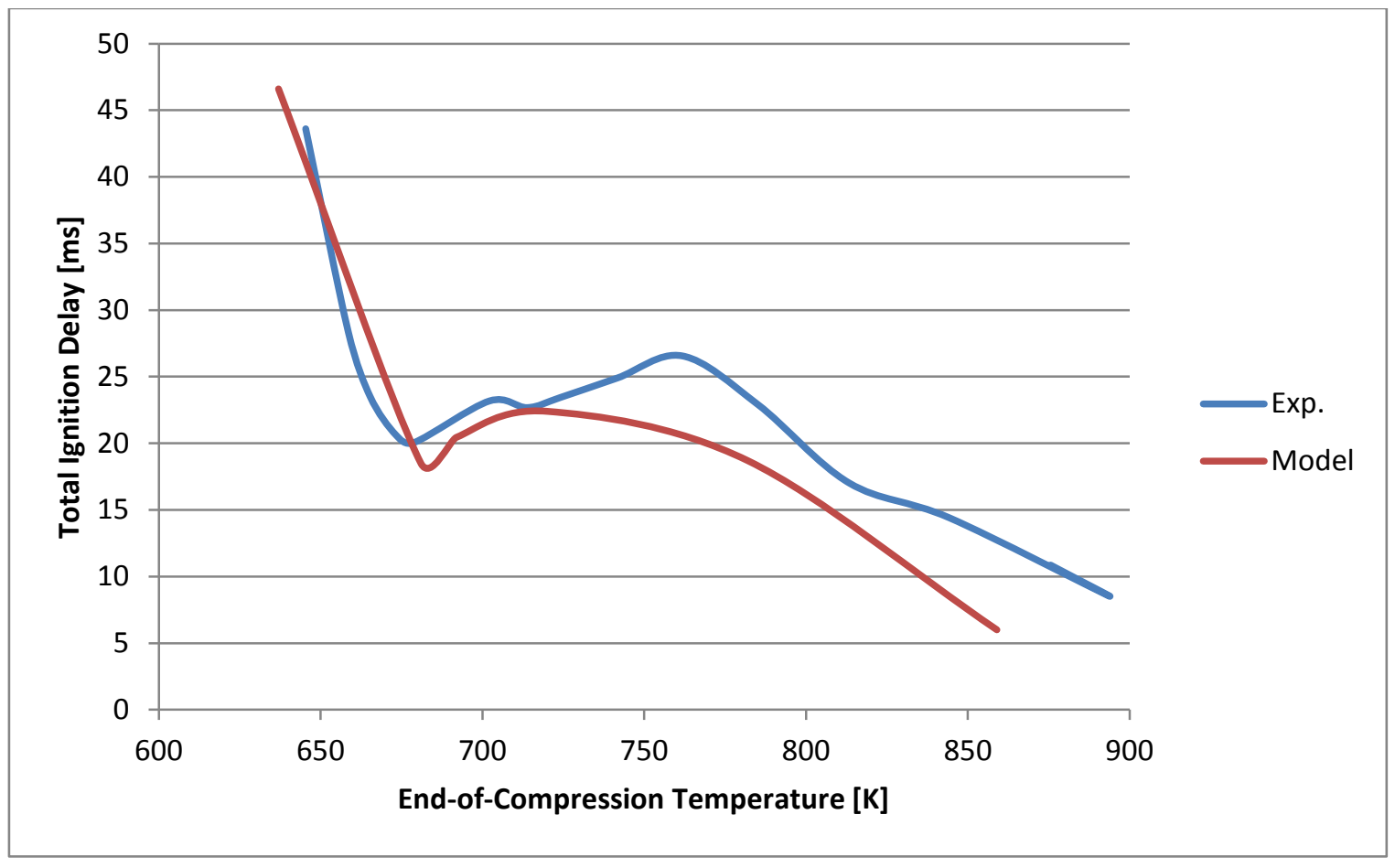

Figure 28 - Configuration 1 total ignition delay, $\Phi=1.12, P_{c}=7 \mathrm{bar}$. 


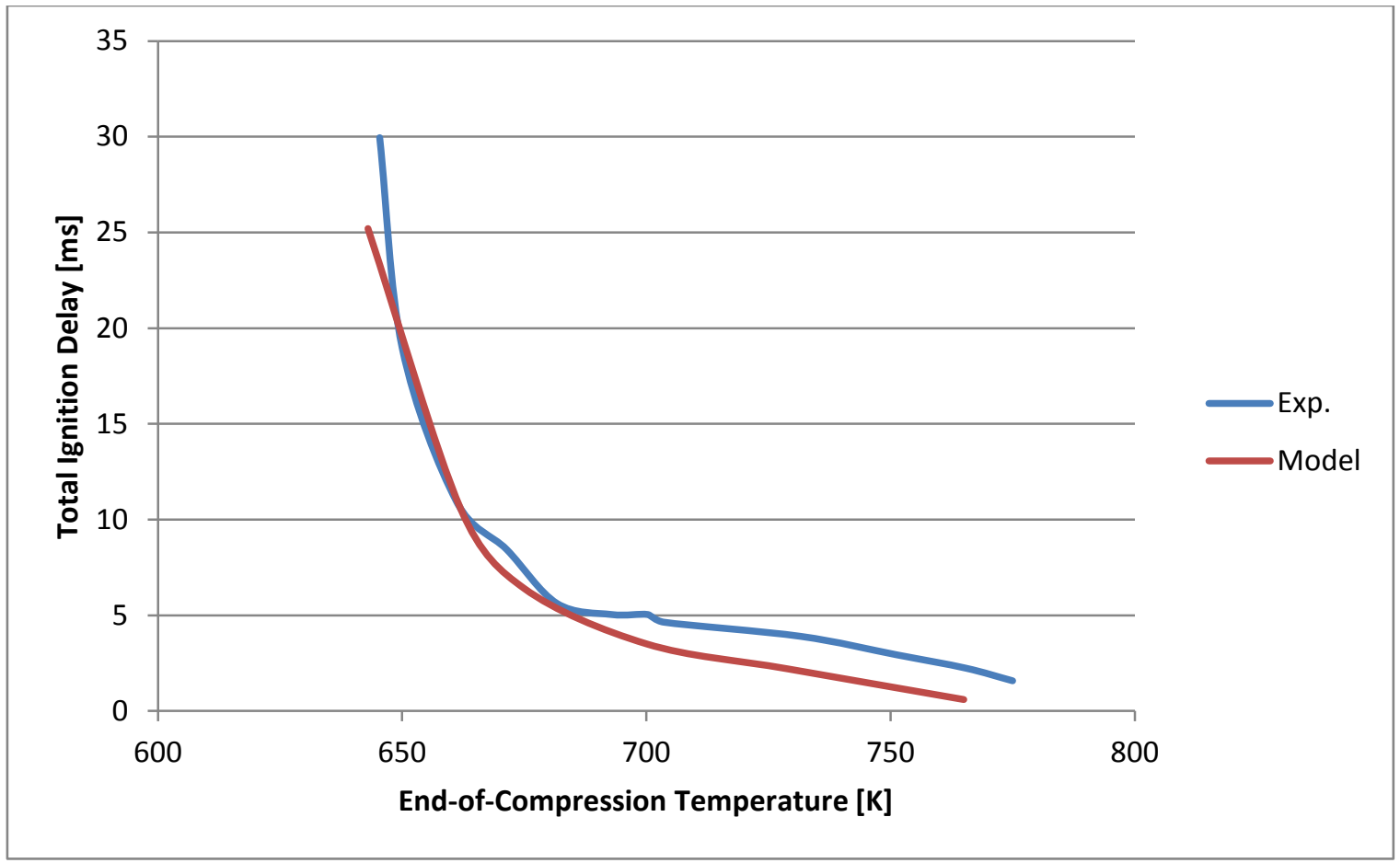

Figure 29 - Configuration 2 total ignition delay, $\Phi=1.12, P_{c}=15 \mathrm{bar}$.

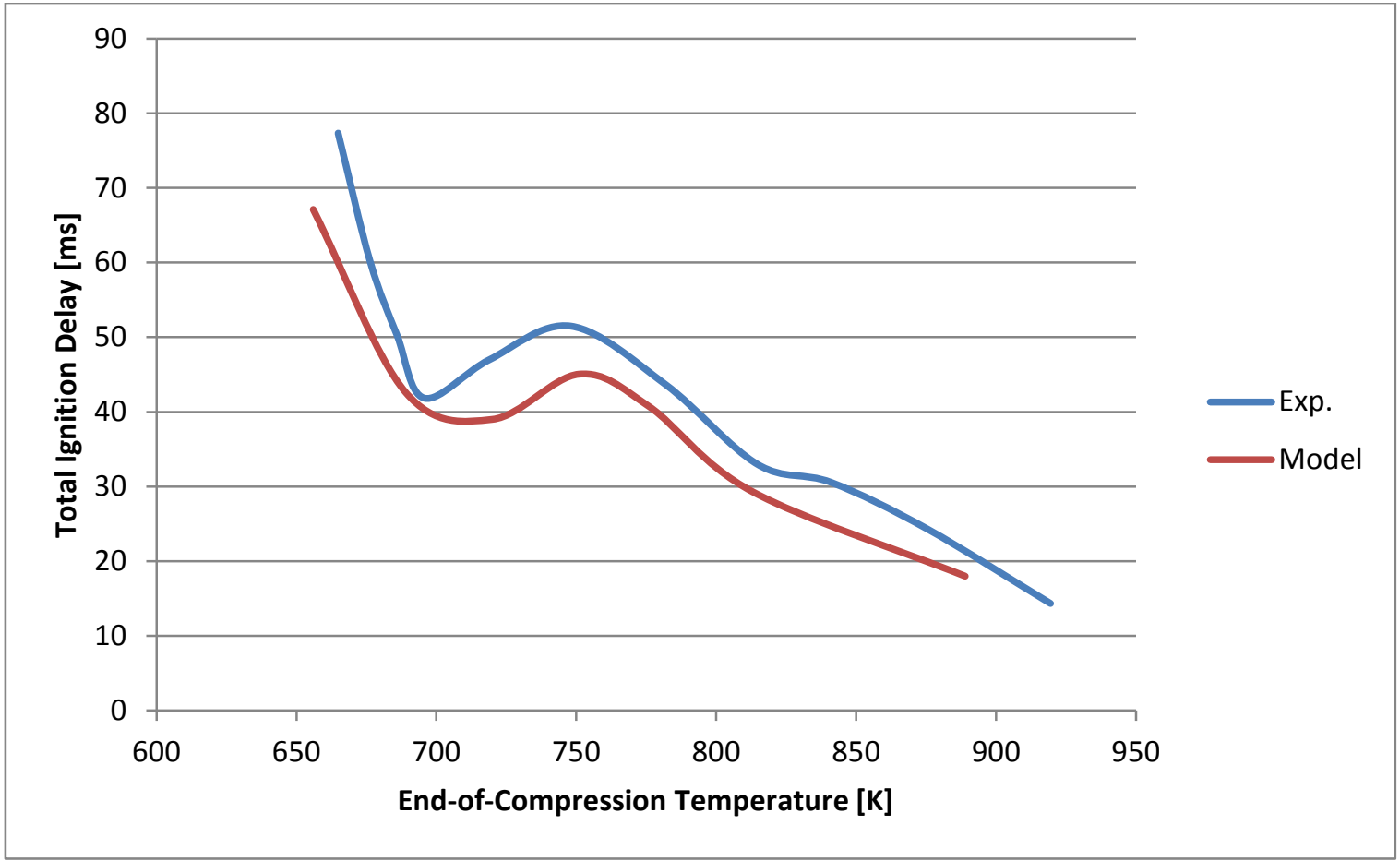

Figure 30 - Configuration 3 total ignition delay, $\Phi=0.76, P_{c}=7$ bar. 


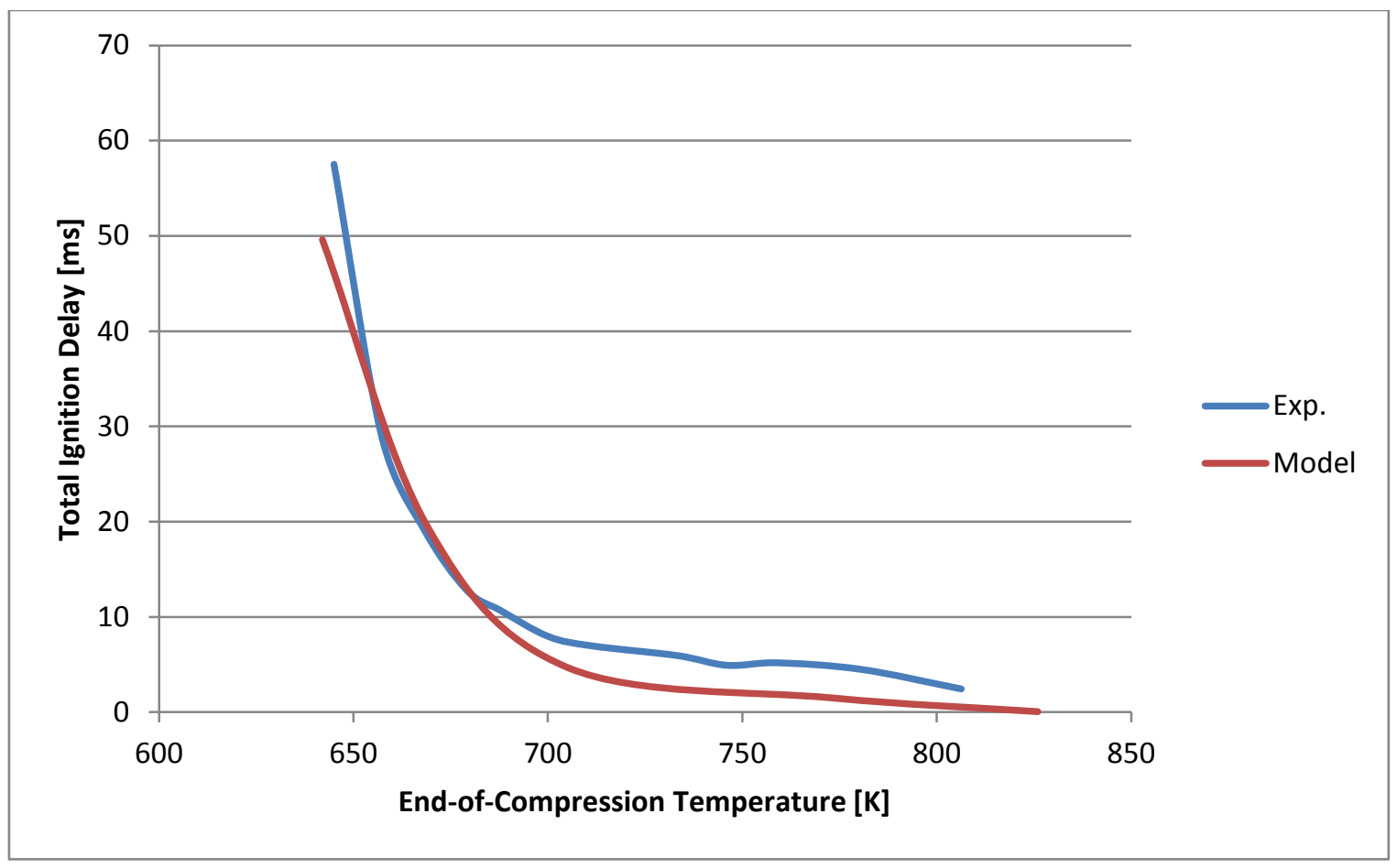

Figure 31 - Configuration 4 total ignition delay, $\Phi=0.76, P_{c}=15$ bar.

Table 6 lists the constants used to tune the Shell model to produce the above ignition delay curves. Figures 28-31 show the total ignition delay: $\tau, \tau_{1}$ and $\tau_{2}$ curves are shown in Appendix B. When comparing Figures 28 and 29 the effect of increasing the pressure from 7 to 15 bar with $\Phi=1.12$ clearly shows a reduced second stage delay presence, the same can be seen when comparing Figures 30 and 31 (7 to 15 bar with $\Phi=0.76)$. In each case the Shell model predictions are better when the second stage delay is less prevalent though Figures 28 and 30 clearly show an ability to predict second stage behaviour. At 15 bar maximum discrepancies of 1.64 and $1.69 \mathrm{~ms}$ are seen for the $\Phi=1.12$ and $\Phi=0.76$ cases, respectively. At 7 bar maximum discrepancies of $6.56 \mathrm{~ms}$ and $7.98 \mathrm{~ms}$ are seen for the $\Phi=1.12$ and $\Phi=0.76$ cases, respectively. Figures 29 and 31 show that the Shell model's accuracy decreases at a certain point $(\sim 675 \mathrm{~K})$ when $T_{c}$ is increased. This 
can be explained by the fact that in the experiments all tests that showed a negative $\tau_{1}$ were given a $\tau_{1}$ of zero instead of the actual value (See Figures B.1, B.3, B.5 and B.7). Physically these cases represent those where ignition occurs before the piston reaches TDC. The slight pressure increase as a result from $\tau_{1}$ can be hard to determine on a pressure-time curve during the compression stroke. In this work the first temporal derivative of pressure was used to find $\tau_{1}$ and it was possible to define the moment when the first stage pressure rise occurred. When comparing model and experimental results, the Shell model predicts lower $\tau$ values then what was measured as $\tau_{1}$ becomes negative. This discrepancy is taken to be a result of different measurement methods and not a result of the model. Still however, the validation results are much better when the case is less dependent on $\tau_{2}$ and better results are expected with higher than stoichiometric equivalence ratios and higher compression ratios. These points of the model must be understood but they are taken to be of less consequence with the current prechamber work because prechambers are typically stiochiometric or richer with high compression ratios. 
Table 6 - Shell model constants for $C_{10.17} H_{19.91}$

\begin{tabular}{|c|c|}
\hline Parameter & Value $[\mathrm{cal} / \mathrm{mol} / \mathrm{cm} / \mathrm{s}]$ \\
\hline$A_{p 1}$ & $4.36 \mathrm{E}+13$ \\
\hline$A_{p 2}$ & $5.08 \mathrm{E}+13$ \\
\hline$A_{p 3}$ & $3.9 \mathrm{E}+13$ \\
\hline$A_{q}$ & $1.1 \mathrm{E}+09$ \\
\hline$A_{b}$ & $4.5 \mathrm{E}+17$ \\
\hline$A_{t}$ & $1.64 \mathrm{E}+10$ \\
\hline$A_{f 1}$ & $7.8 \mathrm{E}-04$ \\
\hline$A_{f 2}$ & $4.1 \mathrm{E}+02$ \\
\hline$A_{f 3}$ & $4.18 \mathrm{E}-01$ \\
\hline$A_{f 4}$ & $2.62 \mathrm{E}+05$ \\
\hline$E_{p 1}$ & $2.93 \mathrm{E}+06$ \\
\hline$E_{p 2}$ & $8.28 \mathrm{E}+07$ \\
\hline$E_{p 3}$ & $3.64 \mathrm{E}+07$ \\
\hline$E_{q}$ & $1.4 \mathrm{E}+08$ \\
\hline$E_{b}$ & $2.07 \mathrm{E}+08$ \\
\hline$E_{t}$ & $3.26 \mathrm{E}+07$ \\
\hline$E_{f 1}$ & $-1.21 \mathrm{E}+07$ \\
\hline$E_{f 2}$ & $-3.01 \mathrm{E}+07$ \\
\hline$E_{f 3}$ & $6.15 \mathrm{E}+07$ \\
\hline$E_{f 4}$ & $1.38 \mathrm{E}+08$ \\
\hline$x_{1}$ & 0 \\
\hline$y_{1}$ & 0 \\
\hline$x_{2}$ & 0 \\
\hline$y_{2}$ & 0 \\
\hline$x_{4}$ & 0 \\
\hline$y_{4}$ & 0 \\
\hline
\end{tabular}




\subsection{Prechamber Simulations}

To demonstrate the function of the combination of the aforementioned and tested models, a prechamber case was built based on the work done at the Laboratory of Industrial Systems (LENI) at École Polytechnique Fédérale de Lausanne (EPFL) [43,50, 9396]. The engine at LENI is a large-bore stationary engine built for cogeneration purposes that runs on either natural gas or biogas. The engine has a bore of $95.25 \mathrm{~mm}$, stroke of $114.3 \mathrm{~mm}$, and a pent-roof combustion chamber with a non-fueled prechamber at the roof apex with a heated surface. Figure 32 shows the position of the prechamber in the cylinder head and Figure 33 shows an enlarged image of the prechamber where the heated walls are highlighted; effectively these heated walls serve the purpose of a glow plug.

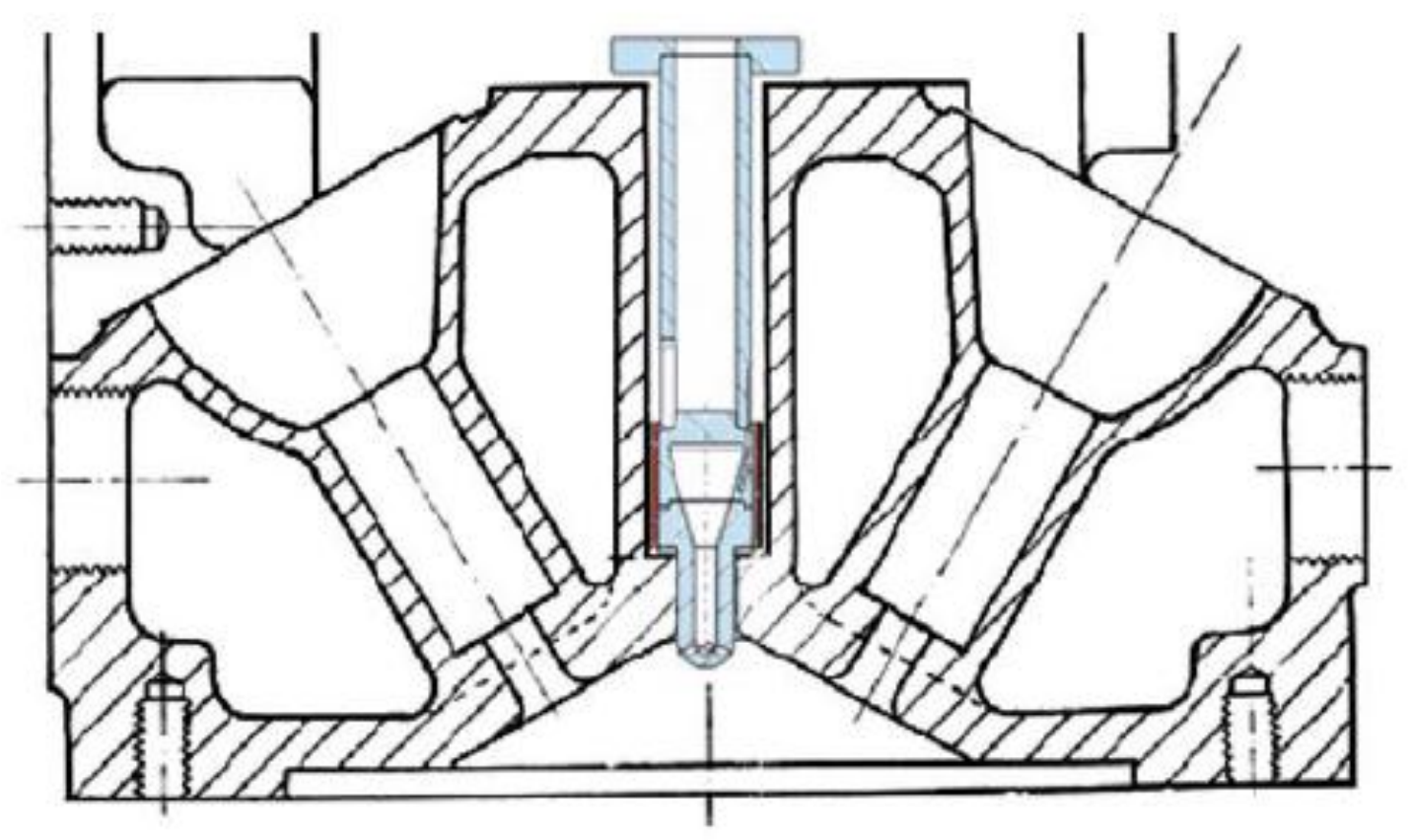

Figure 32 - Position of autoignition prechamber in cylinder head [93]. 

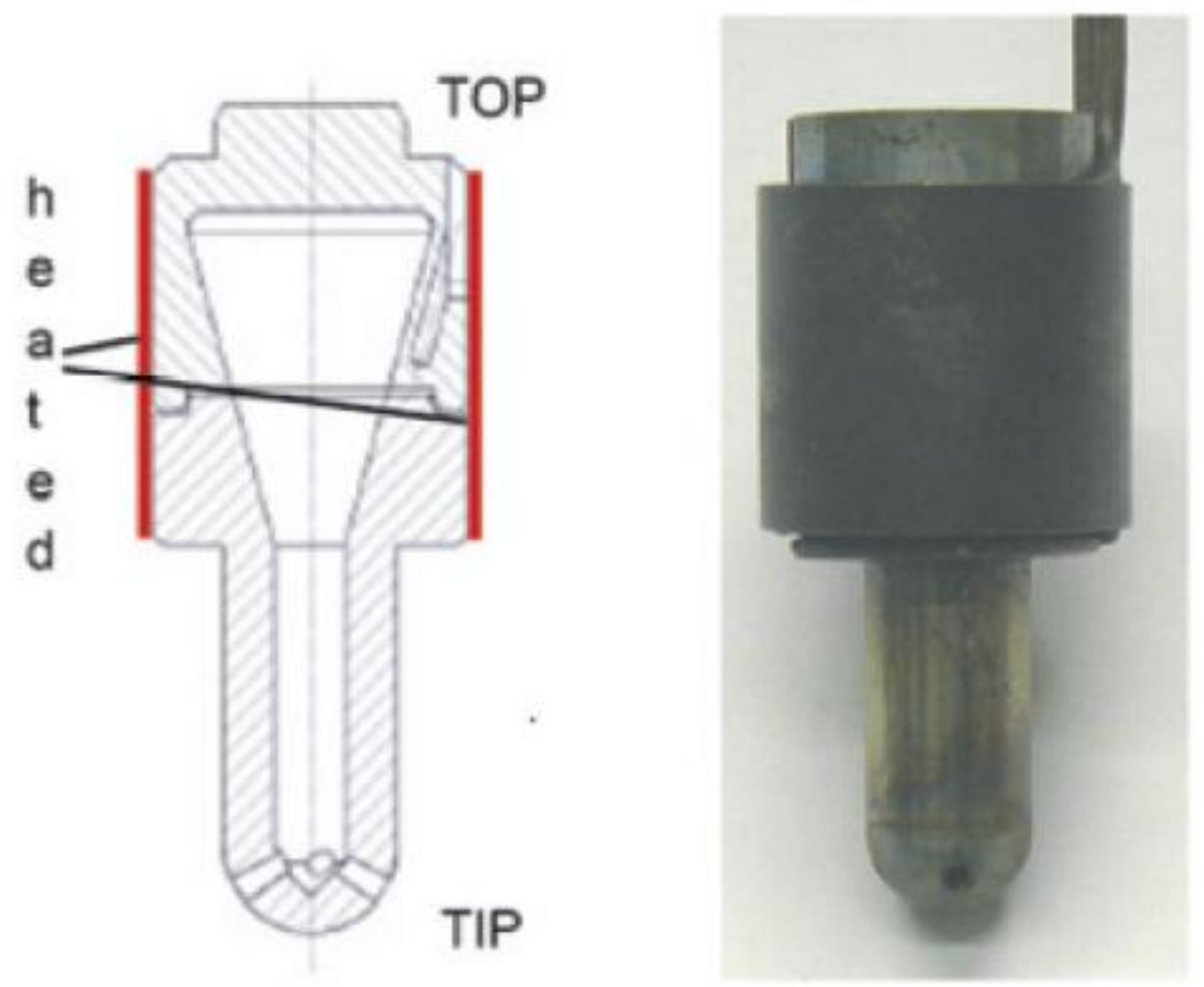

Figure 33 - Prechamber design [43].

The volume of the prechamber is $1630 \mathrm{~mm}^{3}$ with four holes of $1.5 \mathrm{~mm}$ diameter, inclined $58^{\circ}$ in the axial direction, and $10^{\circ}$ in the radial direction. The heating coil dissipates a maximum of $195 \mathrm{~W}$. The engine and prechamber presented here are not used for validation purposes; they are a geometry and operating condition reference chosen because of the large amount of information made public about them. In the simulations presented in this section this configuration is modelled with a premixed main charge and a directly fuelled prechamber with $\mathrm{C}_{10.17} \mathrm{H}_{19.91}$. It is desired to study this fuel's autoignition behaviour in a prechamber environment, regardless of the type of engine in which that prechamber would be used. The conventional use of prechambers has been in a 
reciprocating engine context, for consistency, that context will be used here. It may seem counter-intuitive to be studying an aviation fuel in an automotive context, here though, the method is justified as the physical characteristics related to droplet formation, break-up and evaporation and ignition delay are nonetheless relevant.

The model was built in Catia v5, meshed in ICEM-CFD, and simulated in Fluent. A non-conformal structured mesh was used to model the domain, primarily using the 0-grid function in ICEM-CFD. With the fuel nozzle located at the top of the prechamber, the mesh in the prechamber was sized to achieve similar volume fractions as discussed in Section 4.1. The mesh is shown in Figure 34. Because LES was used the full $360^{\circ}$ model was built where the non-conformal interfaces exist between the nozzles and prechamber.

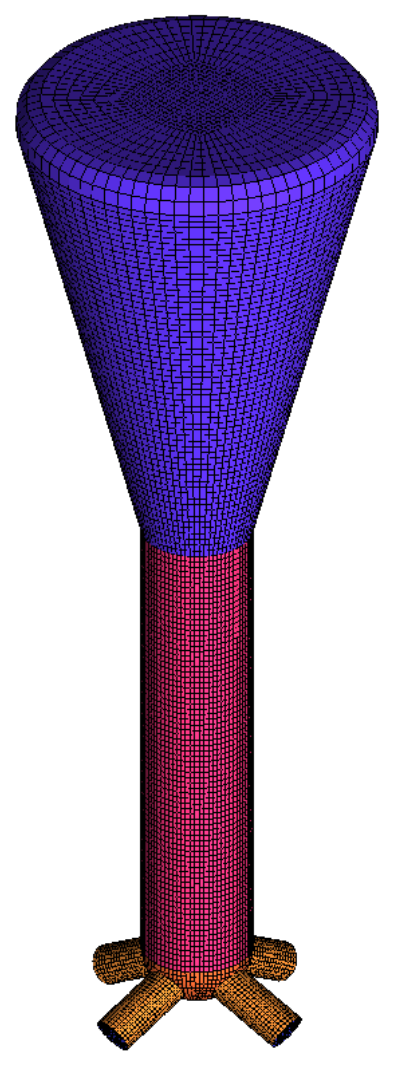

Figure 34-Prechamber mesh where purple section is heated to promote ignition. 
All wall boundaries of the model are given a constant temperature of $376 \mathrm{~K}$ except for the heated prechamber wall as shown in Figures 33 and 34 (purple mesh section in Figure 34), which is heated to $793 \mathrm{~K}$. The nozzle inlets are specified as pressure inlets, temporal volume averaged temperature and pressure profiles taken from simulations of the entire piston/prechamber configuration (not shown here) are used to specify the boundary conditions at these inlets. It is assumed that there is a uniform, lean, $\Phi=0.846$ mixture in the main chamber that enters the prechamber though the pressure inlets at the first time step. No valve motion is considered. The same solver and discretization settings as mentioned in earlier sections were used for this case which is initialized with a constant pressure of $86 \mathrm{kPa}$ and temperature of $376 \mathrm{~K}$ (note that no Exhaust Gas Ricirculation (EGR) is considered) in all mesh zones. The materials are set up as was described in Section 4.2. The in-cylinder parameters were set as shown in Table 7. Simulations are conducted from $\operatorname{BDC}\left(-360^{\circ}\right)$ to $\operatorname{TDC}\left(0^{\circ}\right)$ with a time step of $1 \mathrm{E}-06 \mathrm{~s}$.

Table 7 - In-cylinder parameters.

\begin{tabular}{|l|l|l|}
\hline Crank Shaft Speed [rpm] & Piston Stroke [m] & Connecting Rod Length [m] \\
\hline 1500 & 0.1143 & 0.22225 \\
\hline
\end{tabular}

Fuel is injected into the prechamber at a later time with the strategy being that a rich mixture is created in the prechamber to allow for stable ignition while a lean mixture resides in the cylinder from the intake process. The different spray configurations tested are listed in Table 8. The spray parameters tested are spray timing, spray duration and cone angle, 12 spray configurations in total were simulated. 
Table 8 - Spray parameters.

\begin{tabular}{|l|l|l|l|l|l|l|l|}
\hline Configuration & $\begin{array}{l}\text { Starting } \\
\text { Crank } \\
\text { Angle }\left[^{\circ}\right]\end{array}$ & $\begin{array}{l}\text { Stopping } \\
\text { Crank } \\
\text { Angle }\left[^{\circ}\right]\end{array}$ & $\begin{array}{l}\text { Spray } \\
\text { Duration } \\
\left.{ }^{\circ}\right]\end{array}$ & $\begin{array}{l}\text { Velocity } \\
\text { Magnitude } \\
{[\mathrm{m} / \mathrm{s}]}\end{array}$ & $\begin{array}{l}\text { Cone } \\
\text { Angle } \\
{\left[{ }^{\circ}\right]}\end{array}$ & $\begin{array}{l}\text { Total } \\
\text { Flow } \\
\text { Rate } \\
{[\mathrm{kg} / \mathrm{s}]}\end{array}$ & $\begin{array}{l}\text { Mass } \\
\text { Injected } \\
{[\mathrm{mg}]}\end{array}$ \\
\hline 1 & -90 & -81 & 9 & 150 & 10 & 0.001 & 0.5 \\
\hline 2 & -90 & -72 & 18 & 150 & 10 & 0.001 & 1 \\
\hline 3 & -90 & -81 & 9 & 150 & 30 & 0.001 & 0.5 \\
\hline 4 & -90 & -72 & 18 & 150 & 30 & 0.001 & 1 \\
\hline 5 & -72 & -63 & 9 & 150 & 10 & 0.001 & 0.5 \\
\hline 6 & -72 & -54 & 18 & 150 & 10 & 0.001 & 1 \\
\hline 7 & -72 & -63 & 9 & 150 & 30 & 0.001 & 0.5 \\
\hline 8 & -72 & -54 & 18 & 150 & 30 & 0.001 & 1 \\
\hline 9 & -45 & -36 & 9 & 150 & 10 & 0.001 & 0.5 \\
\hline 10 & -45 & -27 & 18 & 150 & 10 & 0.001 & 1 \\
\hline 11 & -45 & -36 & 9 & 150 & 30 & 0.001 & 0.5 \\
\hline 12 & -45 & -27 & 18 & 150 & 30 & 0.001 & 1 \\
\hline
\end{tabular}

All results are compared with a baseline case where no additional fuel is injected into the prechamber; only the lean mixture from the main chamber is used. In all of the following figures this case will be referred to as 'baseline' where the cases with a spray are referred to by their configuration number listed in Table 8 .

The primary goal is to predict the timing of ignition as a result of varying spray parameters. It is desirable to have the spray evaporate quickly to avoid cooling of the prechamber gases from the fuel. It is also important to have the flame front traverse the domain quickly to provide the largest pressure pulse and to avoid the production of slow forming pollutants $\left(\mathrm{ex} . \mathrm{NO}_{\mathrm{x}}\right.$ ). Also, complete combustion of the fuel is important in terms of maximum energy production and avoiding unburned hydrocarbon and soot emissions. The two graphs that will be used to evaluate these points are volume-averaged pressure and 
equivalence ratio graphs. The pressure graphs directly show timing and magnitude of the pressure rise from combustion, whether ignition is single or multi-stage. The equivalence ratio graphs show the evaporation and burning rates of the fuel as well as the amount of fuel that was oxidized. Figure 35 shows the equivalence ratio profiles for each case tested; the timing of each injection can clearly be seen as the spray curves deviate from the baseline. It is also seen that the average equivalence ratio achieved is higher for cases where the spray occurred earlier, when comparing sprays that injected the same amount of fuel. The burning rate of each case can be estimated by focusing on the rapid decrease in equivalence ratio that occurs after $-40{ }^{\circ} \mathrm{CA}$. A stronger two-stage combustion behaviour is seen with the leaner cases as well as a slower burning rate, while the richer cases show a much sharper decrease in equivalence ratio, indicating stronger single-stage ignition. This dependence of ignition stages on equivalence ratio is expected as shown in the validation cases discussed in Section 4.2. Another feature shown in Figure 35 is the remaining equivalence ratio after combustion. Complete combustion would result in the equivalence ratio becoming zero after combustion. Most of the configurations nearly achieve complete oxidation of the fuel, although some clearly do not; Case 2 for example has an equivalence ratio of roughly 0.4 at $0{ }^{\circ} \mathrm{CA}$. Figure 36 shows the variation in pressure pulse achieved by combustion where the peak occurs between -11 and $-2{ }^{\circ} \mathrm{CA}$ as a result of the varied spray parameters. The magnitude of the pressure peak widely varies with the different spray configurations; Case 9 barely deviates from the baseline case whereas Case 4 produces a pressure rise of over $1 \mathrm{~atm}$. The following sub-sections will describe the effect of varying each spray parameter in more detail. 


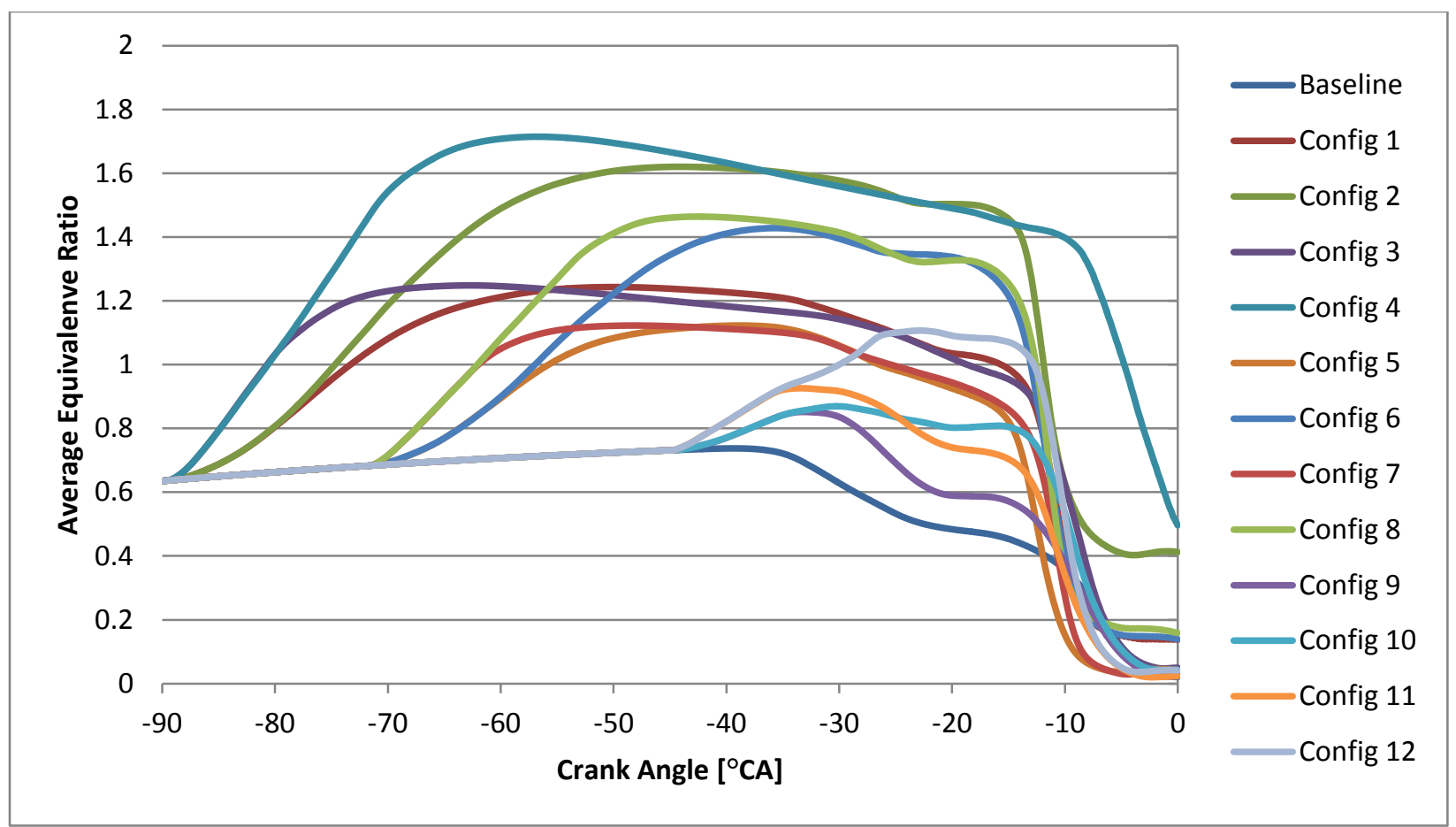

Figure 35-Equivalence ratios for all spray configurations simulated.

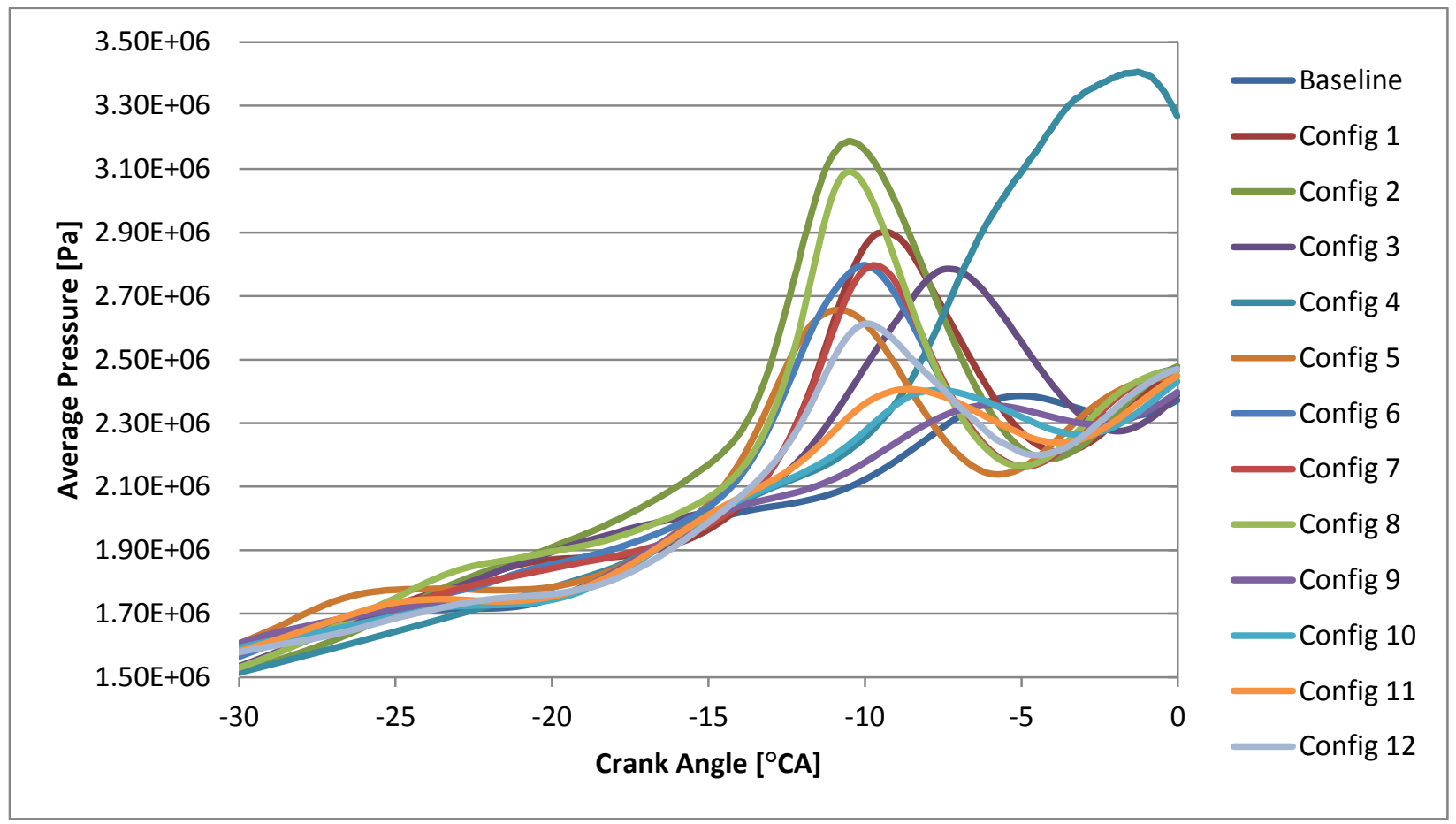

Figure 36-Pressures for all spray configurations simulated. 


\subsection{Spray Timing}

Figures 37-40 compare sprays that were injected at different times with the same duration and cone angle.

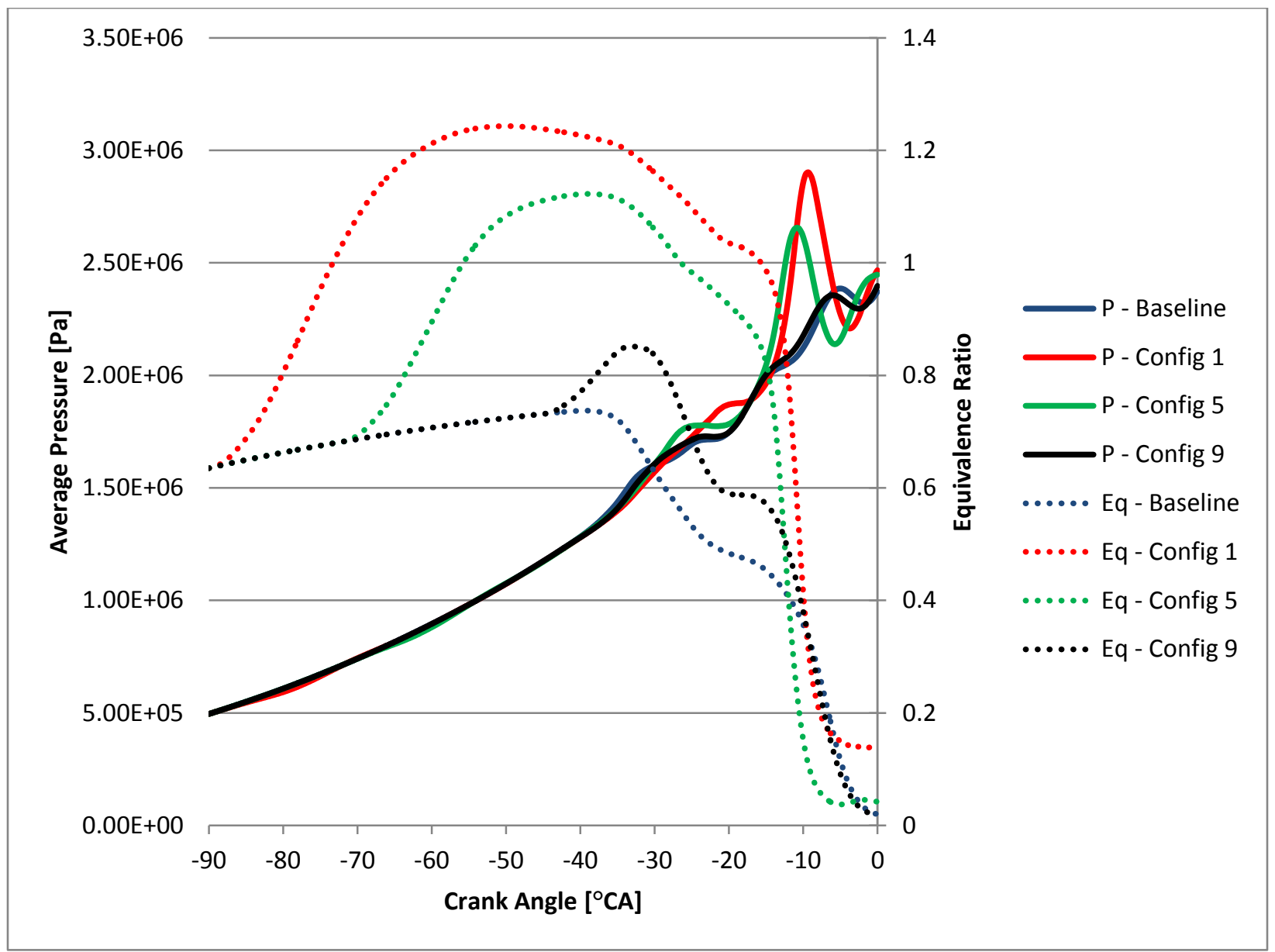

Figure 37- Pressures and equivalence ratios for all sprays lasting $9^{\circ} \mathrm{CA}$ with a $10^{\circ}$ cone.

The sprays in Figure 37 injected into the domain earlier show a larger pressure pulse which occurs later in time, meaning that the timing of the pressure pulse is inversely proportional to the injection timing for Cases 1, 5 and 9. Also, in Case 9 the spray is injected too late to provide an appreciable pressure pulse relative to the baseline case. 


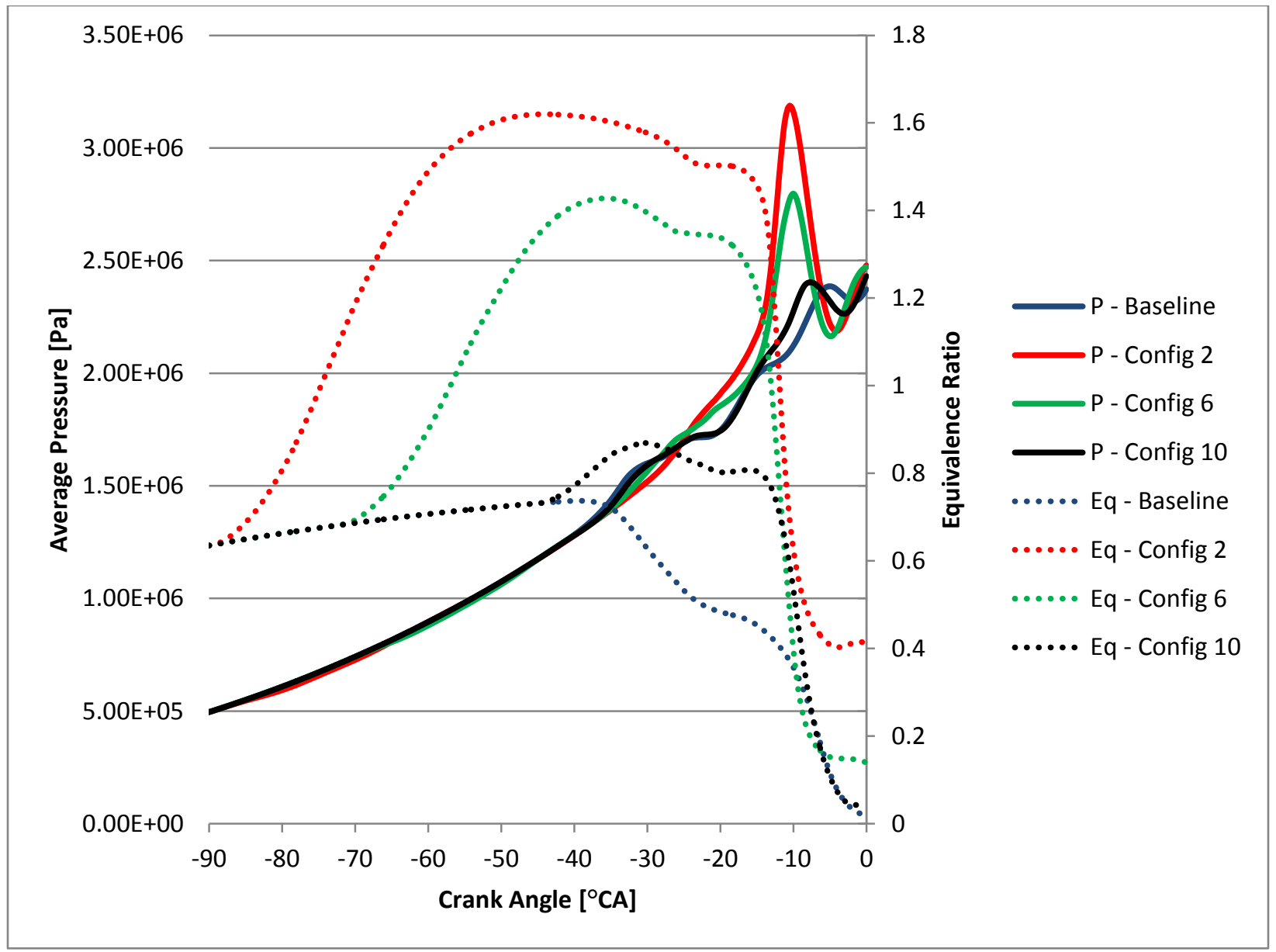

Figure 38-Pressures and equivalence ratios for all sprays lasting $18^{\circ} \mathrm{CA}$ with a $10^{\circ}$ cone.

Figure 38 shows the same cases as in Figure 37 except the injections last twice as long while injecting fuel at the same rate. Again the largest pressure pulse is seen with the case where the spray occurred earliest although less dependence of the pressure pulse timing on injection timing is seen. As expected, the pressure pulse magnitude is much larger when twice as much fuel is injected. The amount of fuel left unburned however, is much larger for the cases in Figure 38 than in Figure 37, meaning that although the pressure pulse is larger, more unburned hydrocarbon and soot emissions are likely. 


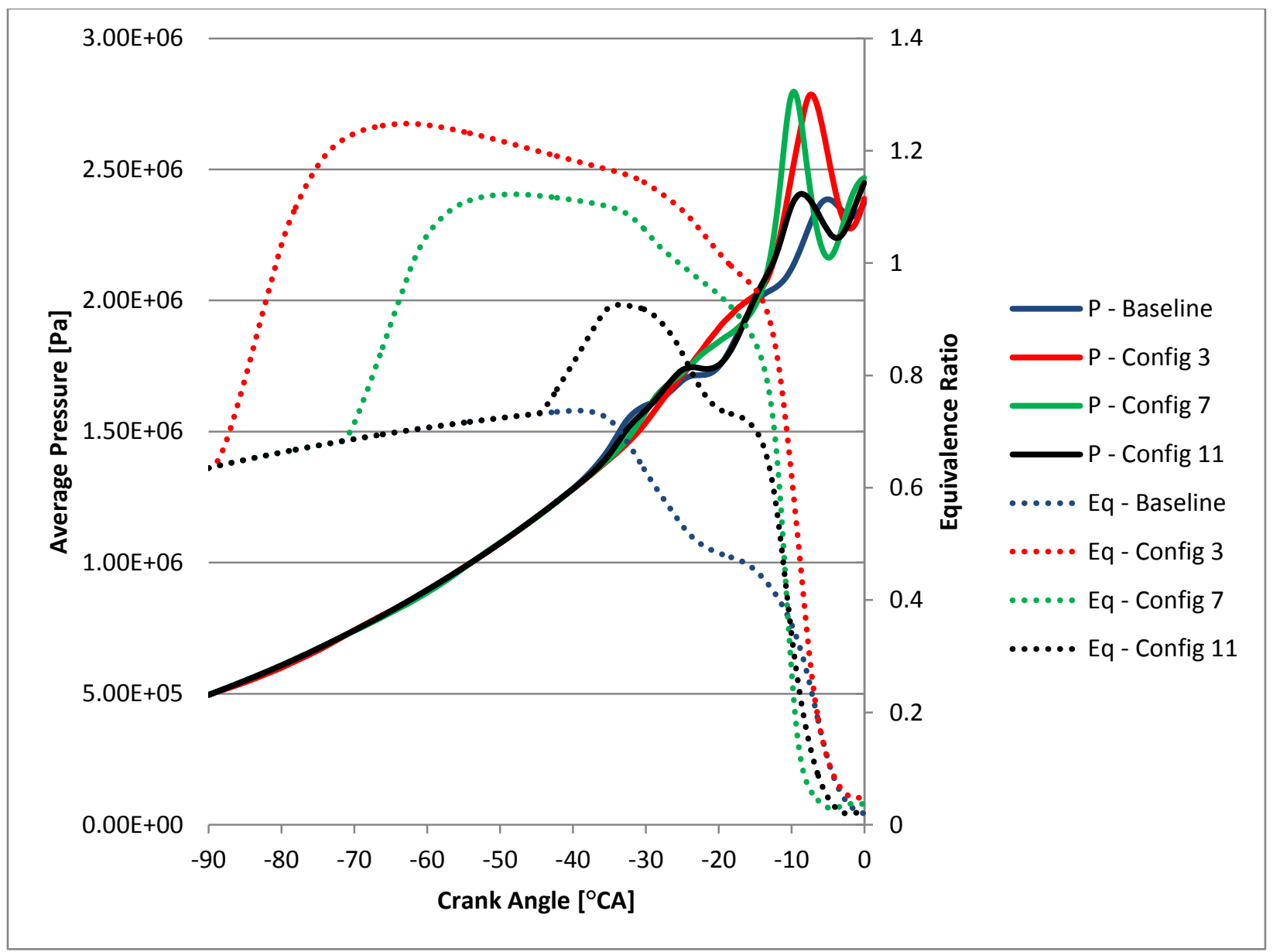

Figure 39- Pressures and equivalence ratios for all sprays lasting $9^{\circ} \mathrm{CA}$ with a $30^{\circ}$ cone.

When the $9^{\circ} \mathrm{CA}$ sprays have their cone angles changed from $10^{\circ}$ to $30^{\circ}$, the pressure pulse magnitude is no longer as dependent on injection timing (Figure 39). This behaviour can be theorized by the notion that fuel is more evenly distributed in the radial direction of the prechamber with a larger cone angle, and a more even equivalence ratio is obtained sooner after the injection begins. There is also less unburned fuel at $0{ }^{\circ} \mathrm{CA}$ which suggests that the larger cone angle helps develop a more even equivalence ratio prior to ignition. 


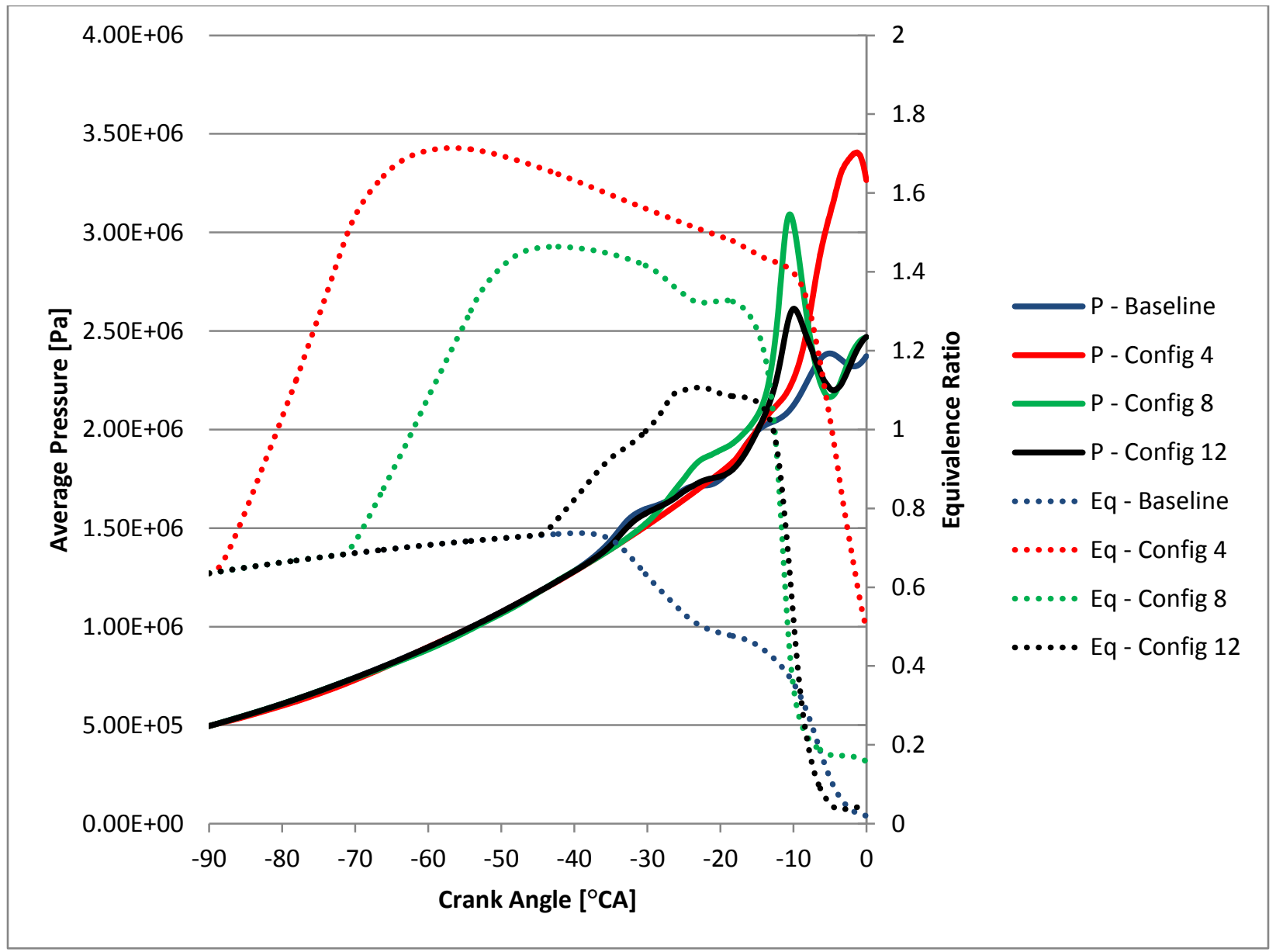

Figure 40-Pressures and equivalence ratios for all sprays lasting $18^{\circ} \mathrm{CA}$ with a $30^{\circ}$ cone.

If the cases shown in Figure 39 (Cases 3, 7 and 11) are given twice the injection time, the cases in Figure 40 are produced (Cases 4, 8 and 12), which unlike those of Figure 39, show a dependence of pressure pulse magnitude on injection timing. Case 4 shows a much longer ignition delay than the other cases. This result can be explained by realizing that Case 4 injects the most fuel at the earliest time with the largest cone angle, so it would be expected that the ignition delay would be longest because the large amount of cold fuel is most evenly distributed in the prechamber, effectively cooling the prechamber gasses and delaying ignition. This hypothesis is confirmed when comparing pre-ignition temporal 
temperature profiles for all cases as shown in Figure 41. The effect of the cold fuel's introduction into the domain on the average temperature can clearly be seen for each spray configuration. Case 4 reduces the domains temperature by the largest amount and this is identified as one reason for the longer ignition delay.

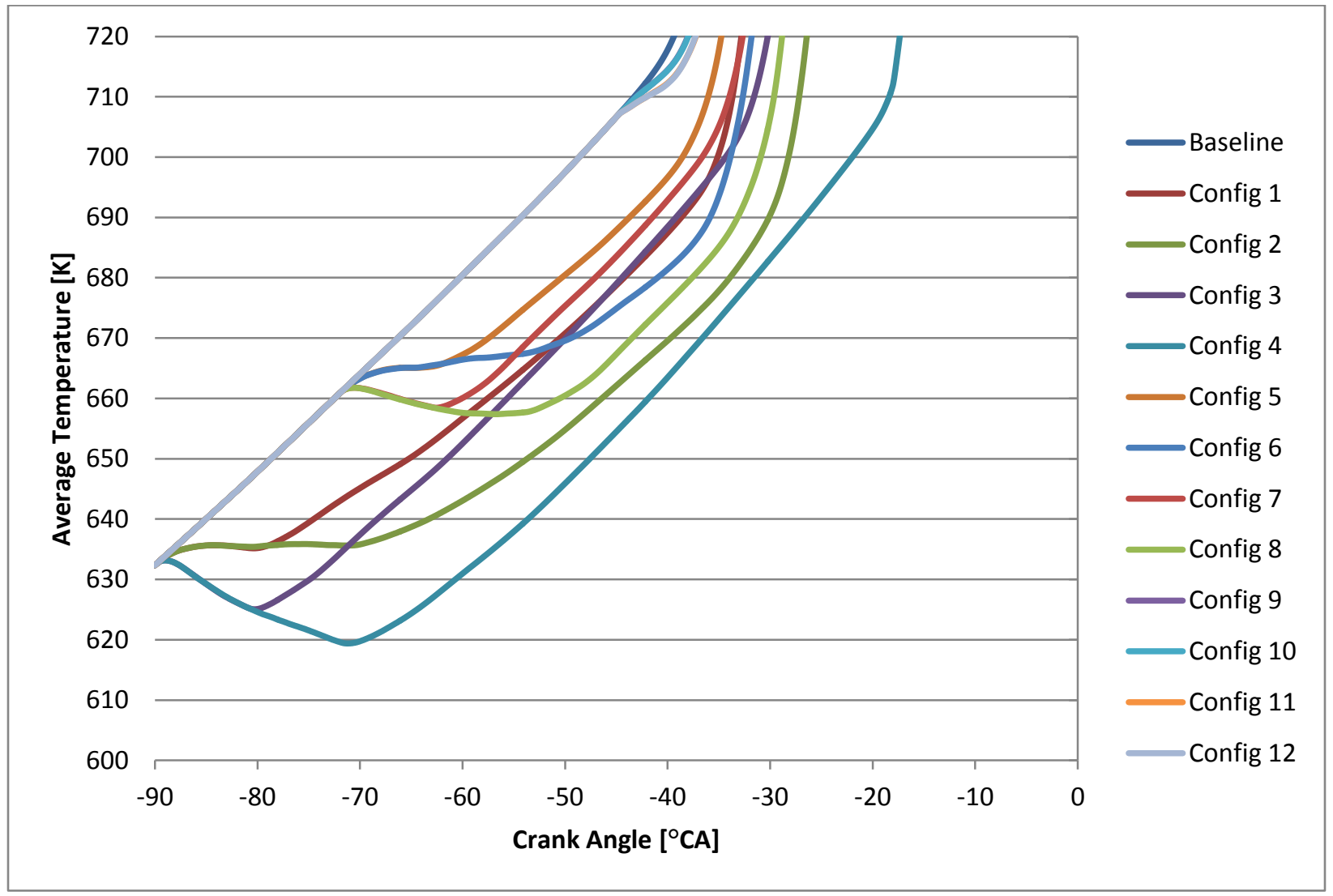

Figure 41-Pre-ignition temperatures for all sprays.

\subsection{Injected Mass}

Figure 42 displays the changes in pressure and equivalence ratio for Cases 1 and 2, where the injection duration was doubled. The same figures for each other comparison of injection duration can be found in Appendix C. 


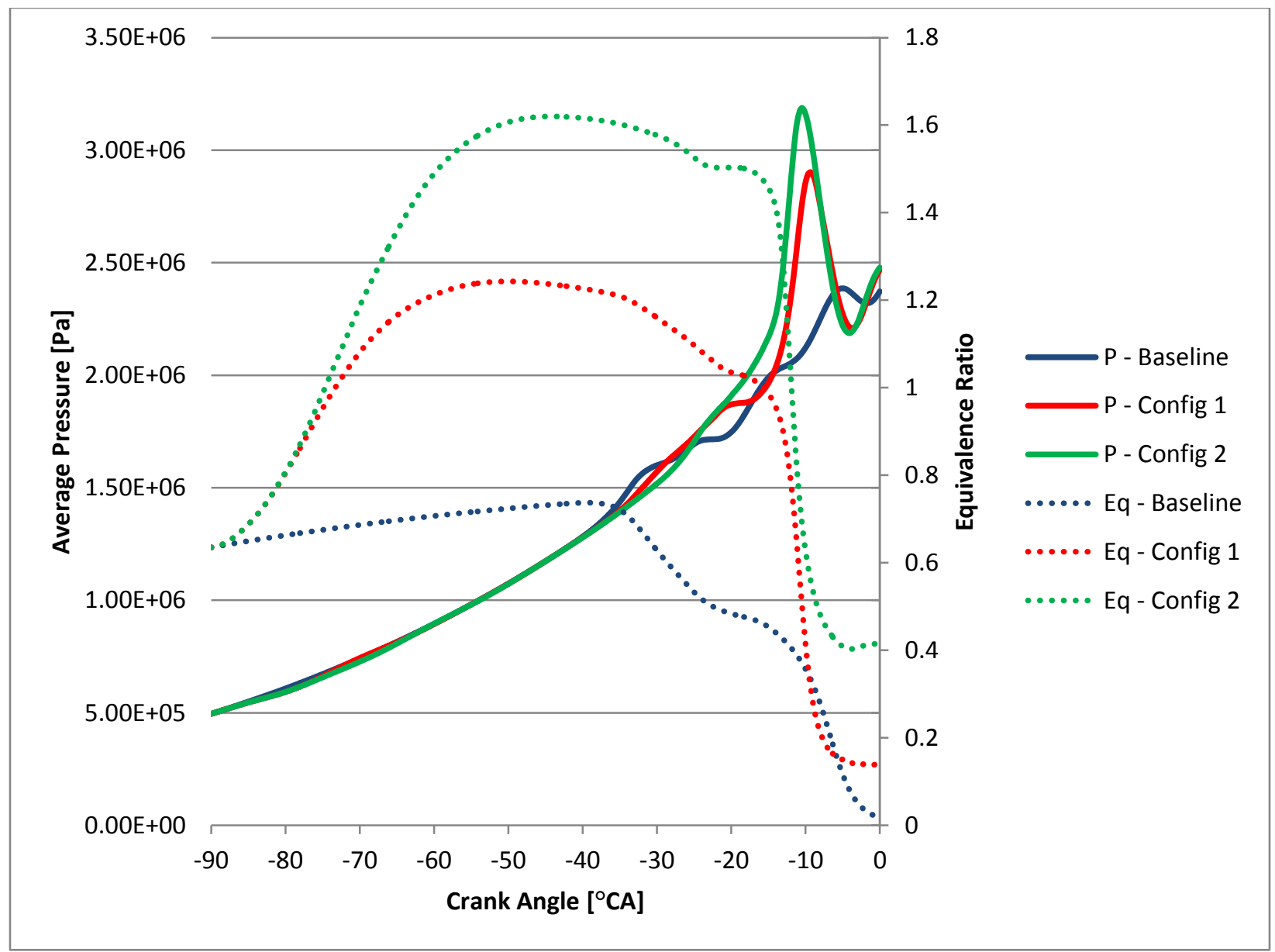

Figure 42-Pressures and equivalence ratios for sprays beginning at $-90^{\circ} \mathrm{CA}$ with a cone angle of $10^{\circ}$ with durations of 9 and $18^{\circ} \mathrm{CA}$.

The effect of injecting twice the amount of fuel into the domain for a given injection timing and cone angle increased the equivalence ratio and subsequently the pressure pulse as would be expected. Also, with an equivalence ratio much larger than 1 it would be expected that some amount of fuel is left unoxidized which can be confirmed in Figure 42 . These trends also occur in each other set of cases where injection duration was varied (See Appendix C). 


\subsection{Spray Angle}

When all other parameters were kept constant and the spray angle was changed from $10^{\circ}$ to $30^{\circ}$ there was a similar effect on all of the cases. For this reason only Cases 1 and 3 (injection beginning at $-90^{\circ} \mathrm{CA}$ with a duration of $9^{\circ} \mathrm{CA}$ and cone angles of $10^{\circ}$ and $30^{\circ}$ ) are included in this section (Figure 43). The figures for the remaining cases are included in Appendix D.

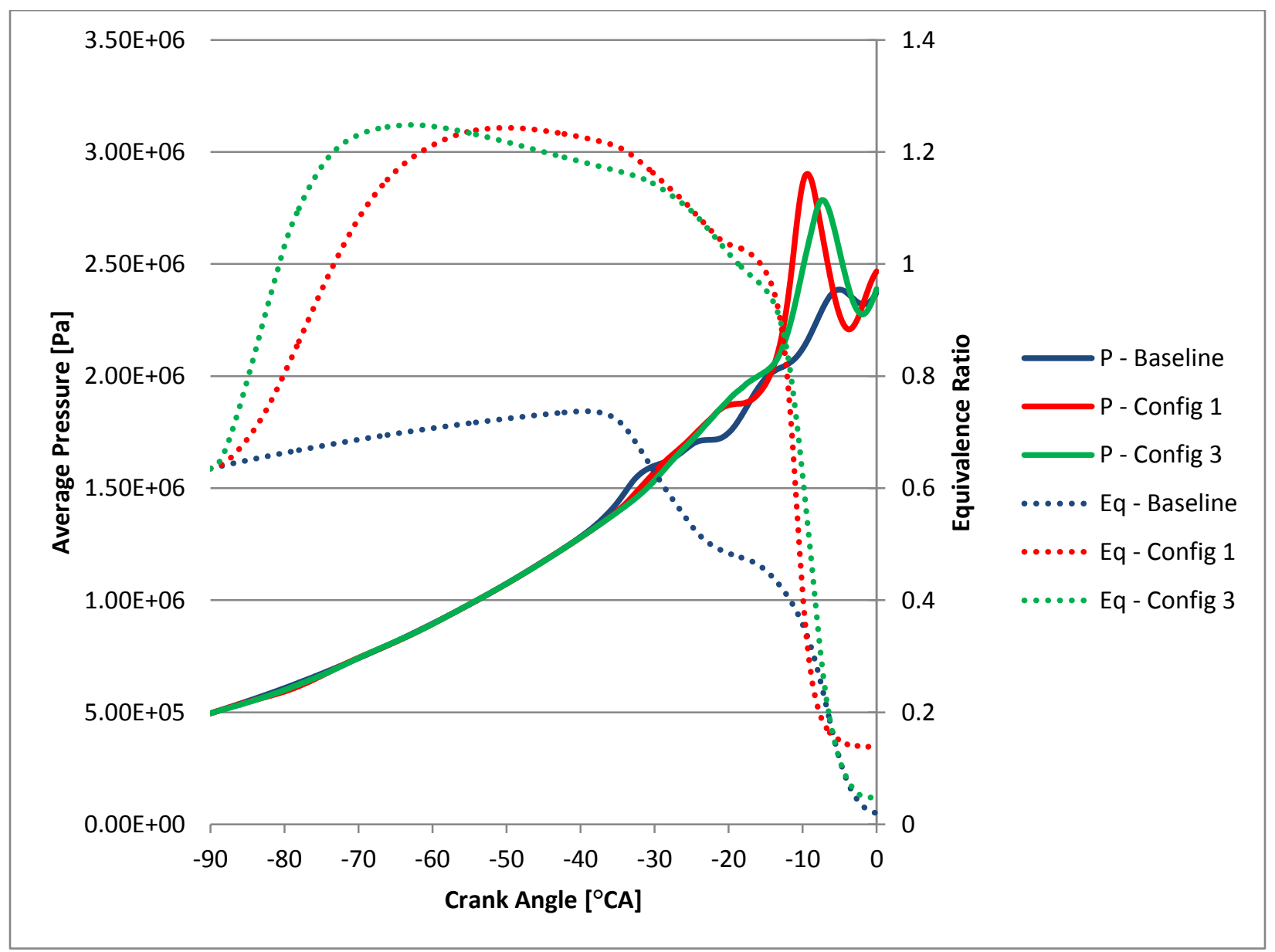

Figure 43- Pressures and equivalence ratios for sprays beginning at $-90^{\circ} \mathrm{CA}$ with a duration of $9^{\circ} \mathrm{CA}$ and cone angles of $10^{\circ}$ and $30^{\circ}$. 
The primary difference noticed when the spray angle is changed is the increased evaporation rate of the fuel which is a mass flux term representing the fuel's entrance into the domain as a vapour after it evaporates from its liquid droplet state. This change is shown in Figure 43 by the larger gradient in the equivalence ratio curves in the -90 to -60 ${ }^{\circ} \mathrm{CA}$ region. The point in time when the maximum equivalence ratio is obtained occurs 0.7 ms earlier. Faster evaporation rates lead to faster transfer of thermodynamic properties between now-vapour fuel and surrounding gas, as opposed to liquid fuel and surrounding gas. This effect can be seen in Figure 41 as each time the spray angle is increased, evaporation increases and the domain reaches a lower average temperature. 


\subsection{Conclusion}

The model produced has a demonstrated ability to predict in-cylinder piston/prechamber processes and would be useful in any situation where indirect or direct fueling or autoignition are present. The final prechamber model presented in Section 5 serves as an example of one such set of processes; the method though is not limited to cases this specific. Prechamber design is known to be highly engine specific and it is of little use to present results of one prechamber and expect that they will be relevant for others. For this reason it is the methodology presented in this work that carries greater merit than the model discussed in Section 5. Only spray timing, spray angle and fuel mass injected were studied over relatively narrow ranges. These studies serve as an example of how the presented methodology could be used in prechamber design where, of course, the parameter studies would be much more extensive. It should be understood though, that the method presented here has some limits of applicability, in validation of both the spray and chemistry sub-model there was a measurable degree of error, as there would be in any such study. Also, the coupling of several models that have different mesh dependencies incorporated some error. In the future it would be ideal to use sub-models that all react to mesh changes similarly. Due to these considerations, the true power of this method is that of a trend predictor more than a tool that can provide accurate values of all parameters over all ranges. Section 5 summarized three such trending studies where sensitivity analyses and trade-offs could be determined, even in cases where the outcome may not be intuitive (as with variations in spray angle). 
In the prechamber arrangement studied here it was found that spray parameters had a large influence on the subsequent combustion and that the inclusion of a prechamber spray could shift the ignition from two-stage to single-stage. It was also found that the cooling of prechamber gasses from the cold spray had a large influence on ignition timing and that injecting a larger amount of fuel, if injected cold, could lead to a longer delay. This is an interesting notion because it is the purpose of the prechamber to burn rich as to maximize ignition stability but the results shown in this work highlight that simply introducing more fuel could produce the opposite result in certain circumstances, either due to cooling of the domain from the fuel or by creating a composition too rich to sustain combustion. In each set of cases where spray angle was varied a faster evaporation rate was seen with an increased spray angle. This notion is very important as the fuel evaporation rate is a very important parameter that is influenced by many variables, the spray angle being one of those variables that can be controlled with the injector. When the mass of fuel injected was doubles larger pressure rises were seen in all cases, but so was the amount of unburned fuel. It must be stated that this unburned fuel would travel to the main chamber where it could then be oxidized, these processes though were not studied in this work.

Further work to the model could be done in a number of areas. Firstly, the chemistry model as is has no capability of predicting emissions such as soot or $\mathrm{NO}_{\mathrm{x}}$, and although the model can predict the mass of unburned fuel, $\mathrm{CO}_{2}$ and $\mathrm{CO}$, these are likely incorrect. Their only function within the Shell model is to predict the heat release to fit experimental pressure curves. The pressure attained from the heat release of the formation of $\mathrm{CO}_{2}, \mathrm{CO}$ and $\mathrm{H}_{2} \mathrm{O}$ was validated but the individual concentrations of those species were not. In the 
Shell model the ratio of $\mathrm{CO}_{2}$ to $\mathrm{CO}$ is held constant and set with $\chi$ (See Equation 77). The modelling of any scenario where $\chi$ would vary from its given constant value would lead to incorrect $\mathrm{CO}_{2}$ to $\mathrm{CO}$ predictions even though the pressure would be validated. If emissions were to be predicted, those predictions would likely not be possible without major revisions to the chemical model. Given the transient nature of in-cylinder CFD problems requiring small time steps, it is not likely that in the near future the use of large chemical mechanisms will be computationally efficient enough to assist in fast design cycles. Thus reduced chemistry models are still required and one that uses a reasonably detailed mechanism so that it can predict two-stage ignition and emissions without too greatly affecting CFD computation times would be ideal.

Another area that warrants further research is the spray model. Currently, the Euler-Lagrange formulation is commonplace but its well-known mesh dependencies are limiting. Given the mesh requirements of LES, it is intuitive to conclude that the Euler-Euler model would be more appropriate for a LES spray solution because both the fluid and spray models would respond similarly to mesh density increases. The Euler-Euler model though is more difficult to incorporate, especially in dilute regions of the spray, thus a hybrid model wherein the Euler-Euler model is used in the spray core and the EulerLagrange model is used in the spray periphery would be useful. Such a model would have the added potential of modelling primary breakup and in-nozzle phenomena - two areas for which large assumptions were made in this work. 


\section{Appendices}

Appendix A - Shell Model Chemistry Source Terms

$$
\begin{aligned}
& \frac{d\left[C_{n} H_{2 m}\right]}{d t}=-k_{q}\left[C_{n} H_{2 m}\right]\left[O_{2}\right]-\frac{\Lambda+1}{m} k_{p}\left[C_{n} H_{2 m}\right]^{\frac{\Lambda+1}{m}}\left[O_{2}\right]^{(\Lambda+1) p} \\
& \frac{d\left[O_{2}\right]}{d t}=-k_{q}\left[C_{n} H_{2 m}\right]\left[O_{2}\right]-p(\Lambda+1) k_{p}\left[C_{n} H_{2 m}\right]^{\frac{\Lambda+1}{m}}\left[O_{2}\right]^{(\Lambda+1) p} \\
& \frac{d\left[H_{2} O\right]}{d t}=k_{p}[R]\left[C_{n} H_{2 m}\right]^{\frac{\Lambda+1}{m}}\left[O_{2}\right]^{(\Lambda+1) p} \\
& \frac{d\left[\mathrm{CO}_{2}\right]}{d t}=(1-\chi) \frac{n}{m} k_{p}[R]\left[C_{n} H_{2 m}\right]^{\frac{\Lambda+1}{m}}\left[O_{2}\right]^{(\Lambda+1) p} \\
& \frac{d[C O]}{d t}=\chi \frac{n}{m} k_{p}[R]\left[C_{n} H_{2 m}\right]^{\frac{\Lambda+1}{m}}\left[O_{2}\right]^{(\Lambda+1) p} \\
& \frac{d[R]}{d t}=2 k_{q}\left[C_{n} H_{2 m}\right]\left[O_{2}\right]+2 k_{b}[B]-f_{3} k_{p}[R]-2 k_{t}[R]^{2} \\
& \frac{d[Q]}{d t}=f_{4} k_{p}[R]\left[C_{n} H_{2 m}\right]^{\frac{\Lambda+1}{m}}\left[O_{2}\right]^{(\Lambda+1) p}-f_{2} k_{p}[R][Q] \\
& \frac{d[B]}{d t}=f_{1} k_{p}[R]\left[C_{n} H_{2 m}\right]^{\frac{\Lambda+1}{m}}\left[O_{2}\right]^{(\Lambda+1) p}+f_{2} k_{p}[R][Q]-k_{b}[B] \\
& \frac{d[T]}{d t}=f_{3} k_{p}[R]+2 k_{t}[R]^{2}
\end{aligned}
$$




\section{Appendix B - First and Second Stage Delay Simulations Results}

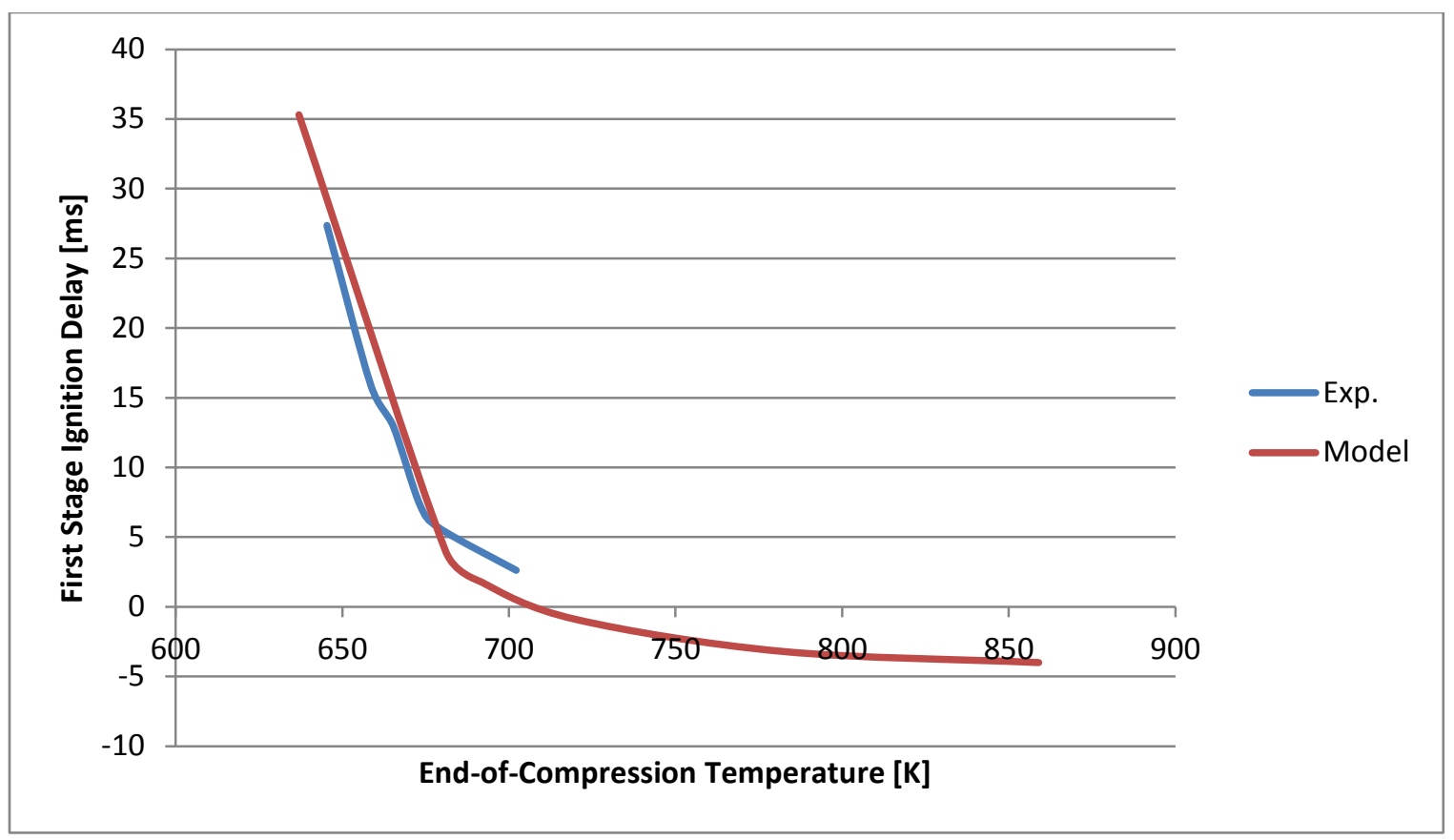

Figure B.1 - Configuration 1 first stage ignition delay.

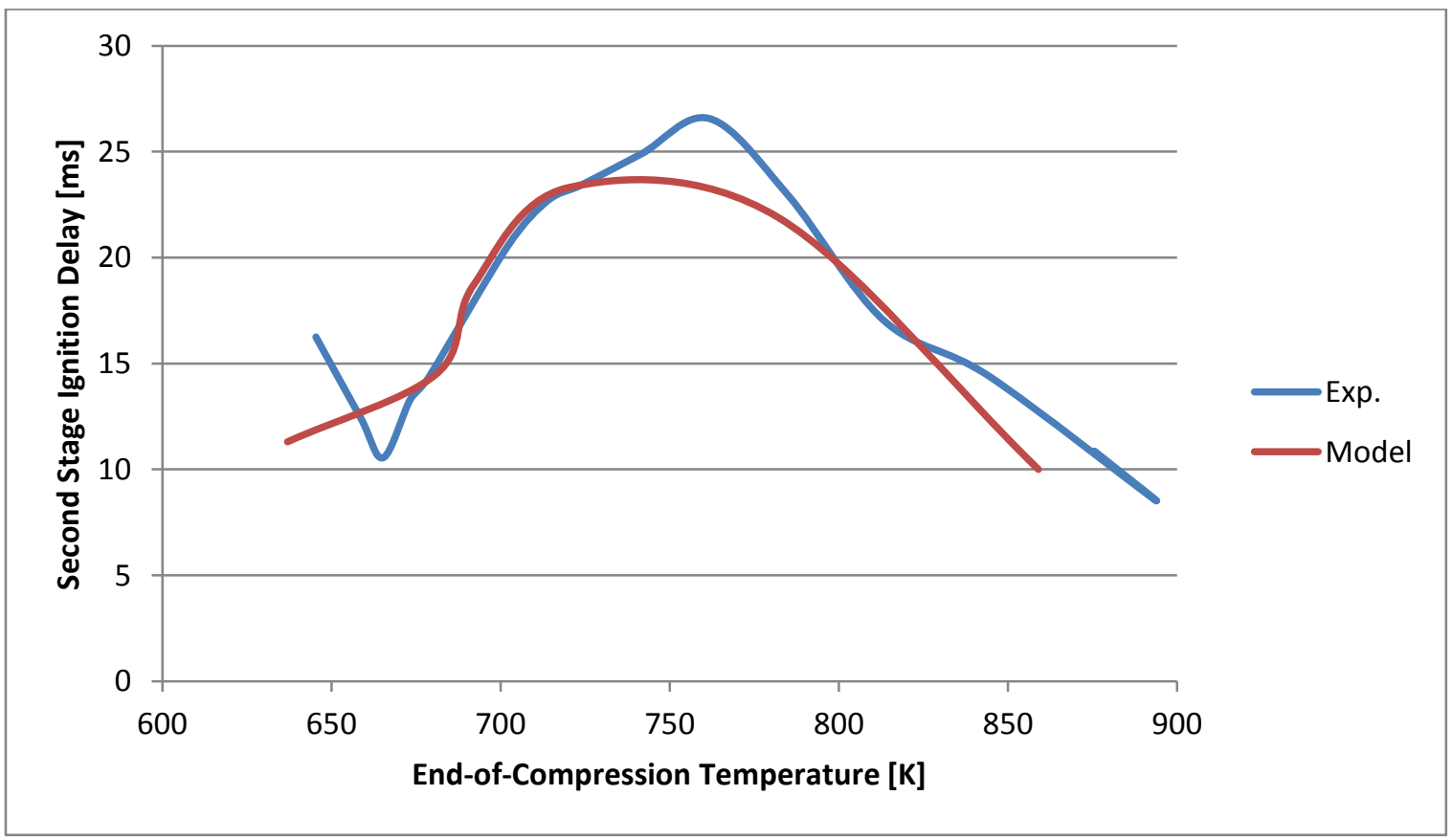

Figure B.2 - Configuration 1 second stage ignition delay. 


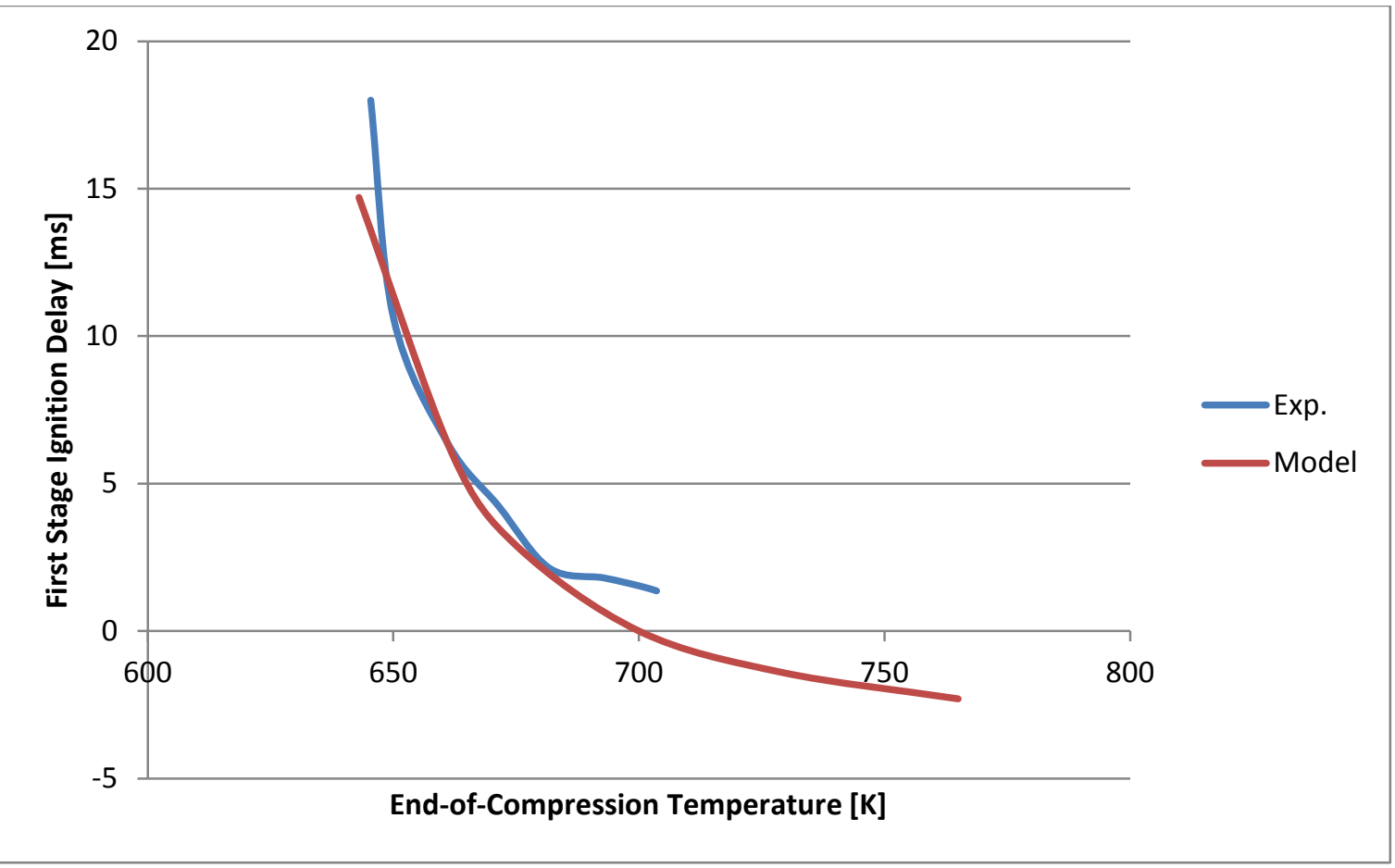

Figure B.3 - Configuration 2 first stage ignition delay.

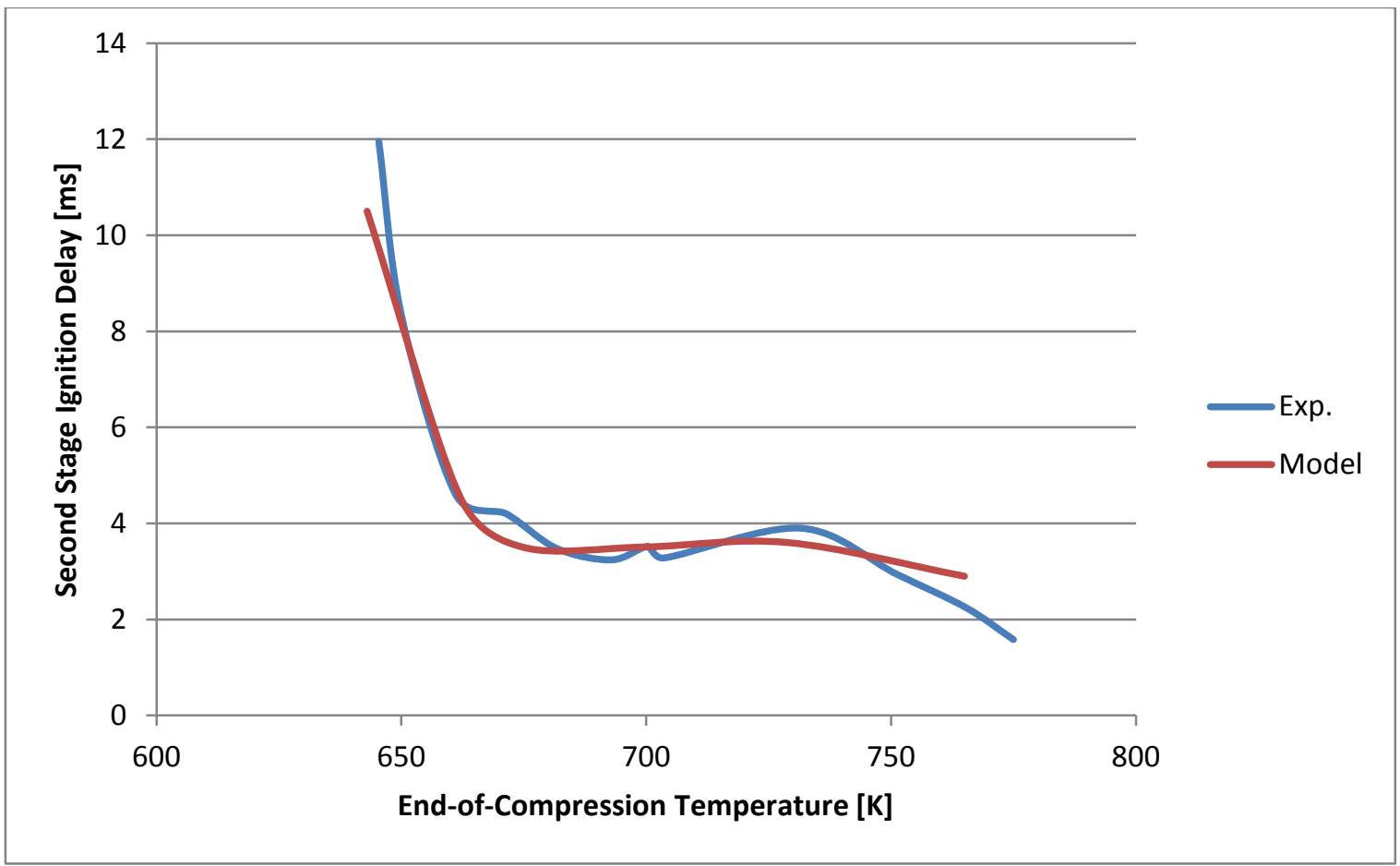

Figure B.4 - Configuration 2 second stage ignition delay. 


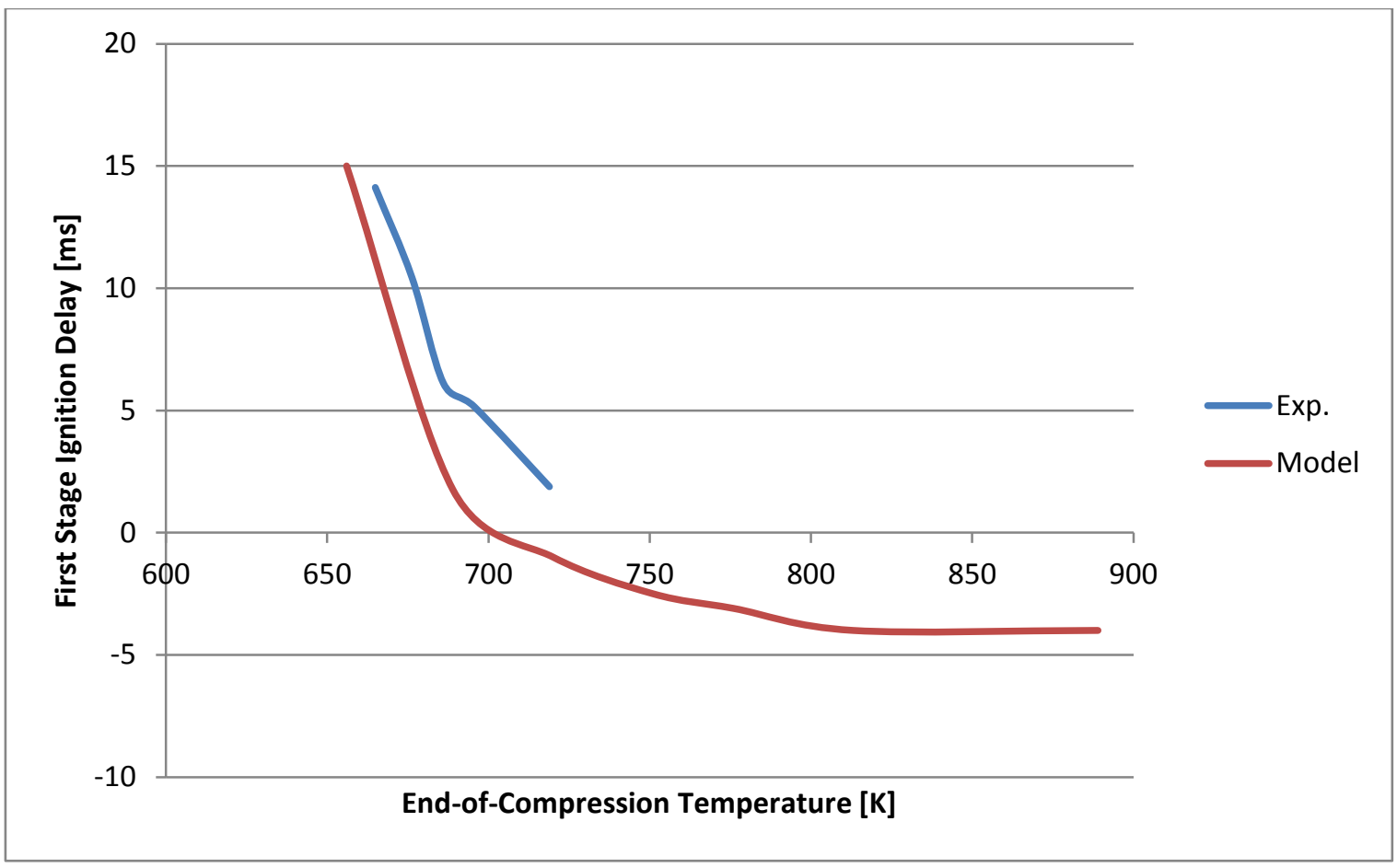

Figure B.5 - Configuration 3 first stage ignition delay.

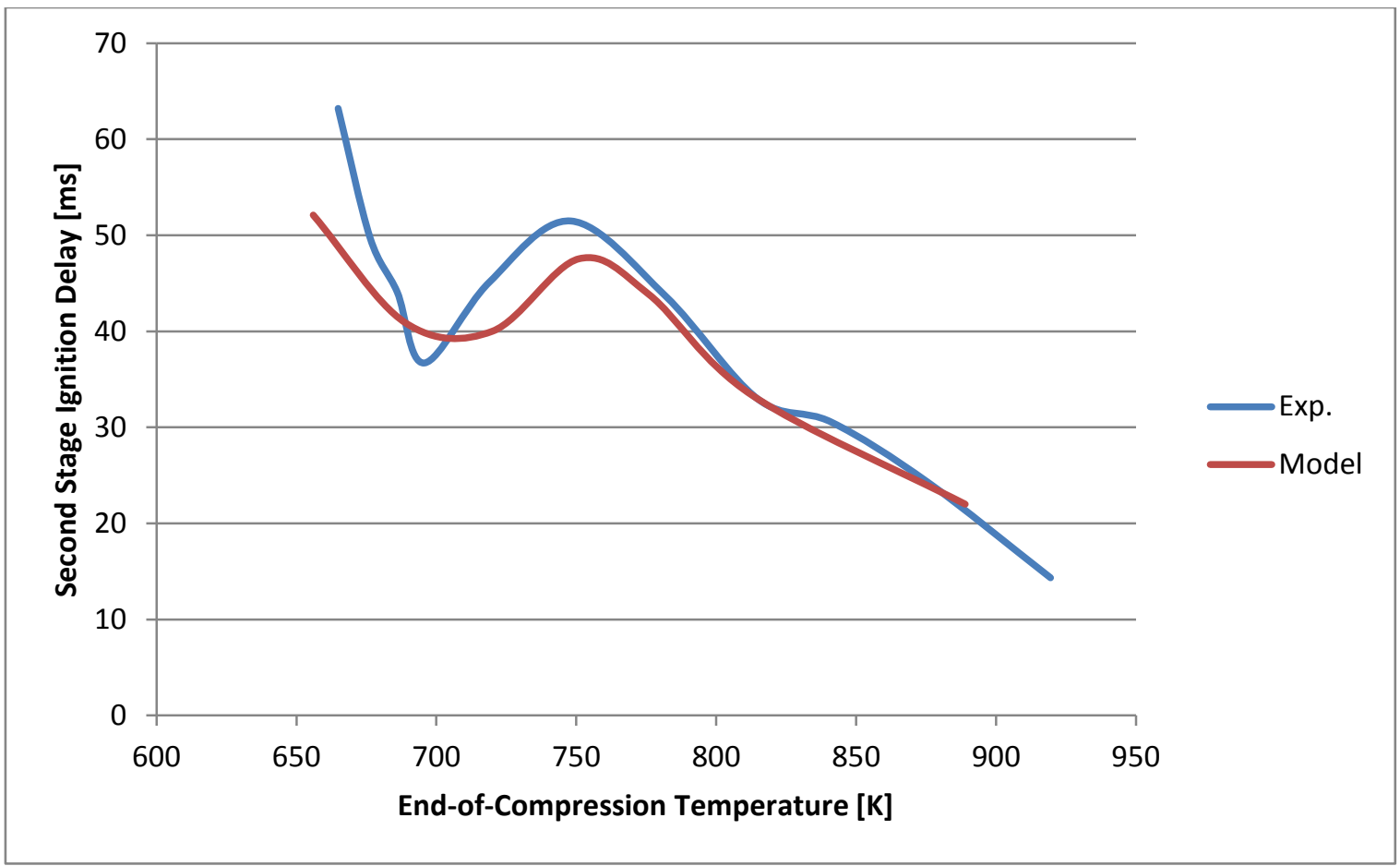

Figure B.6 - Configuration 3 second stage ignition delay. 


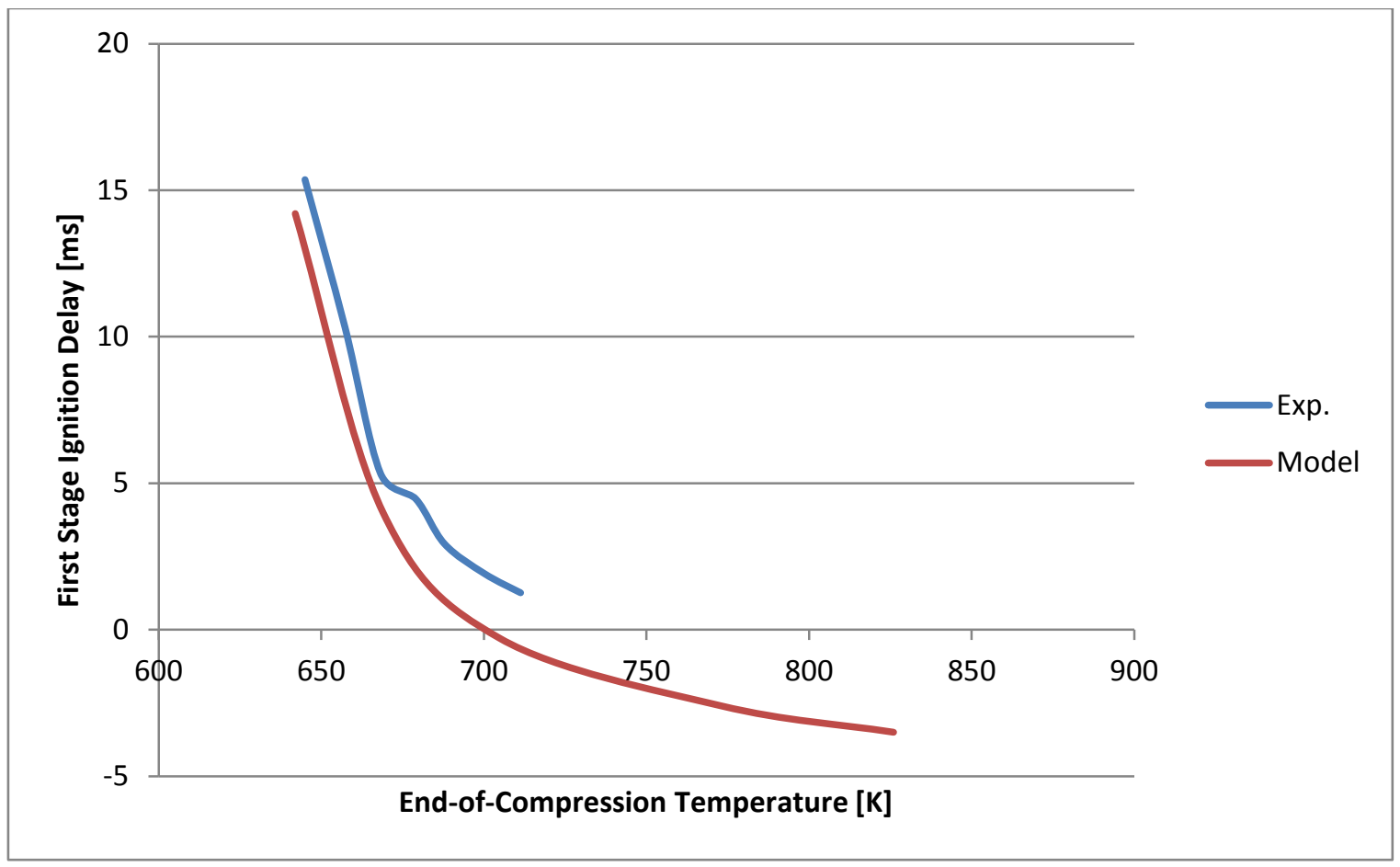

Figure B.7 - Configuration 4 first stage ignition delay.

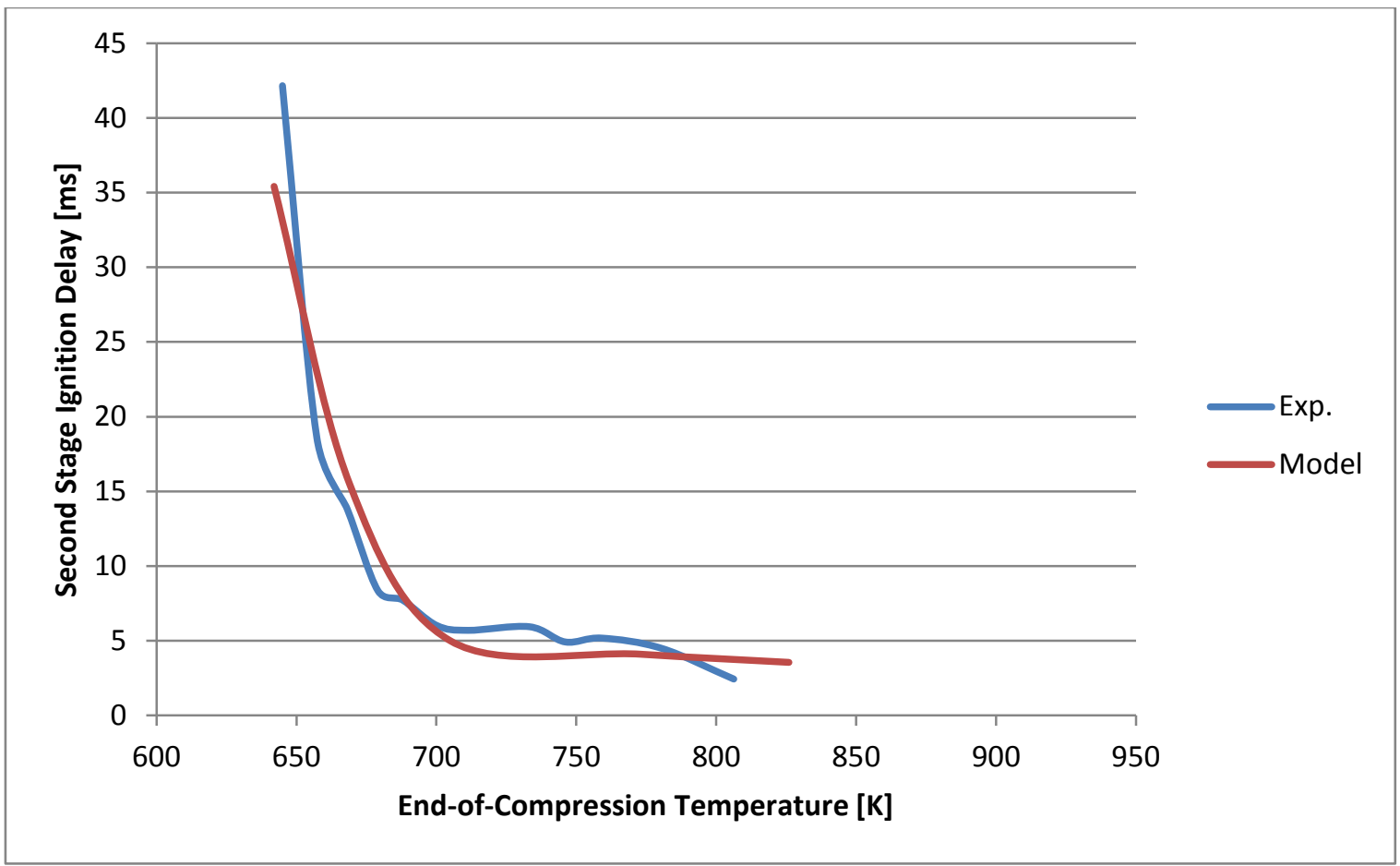

Figure B.8 - Configuration 4 second stage ignition delay. 


\section{Appendix C - Injected Mass Simulation Results}

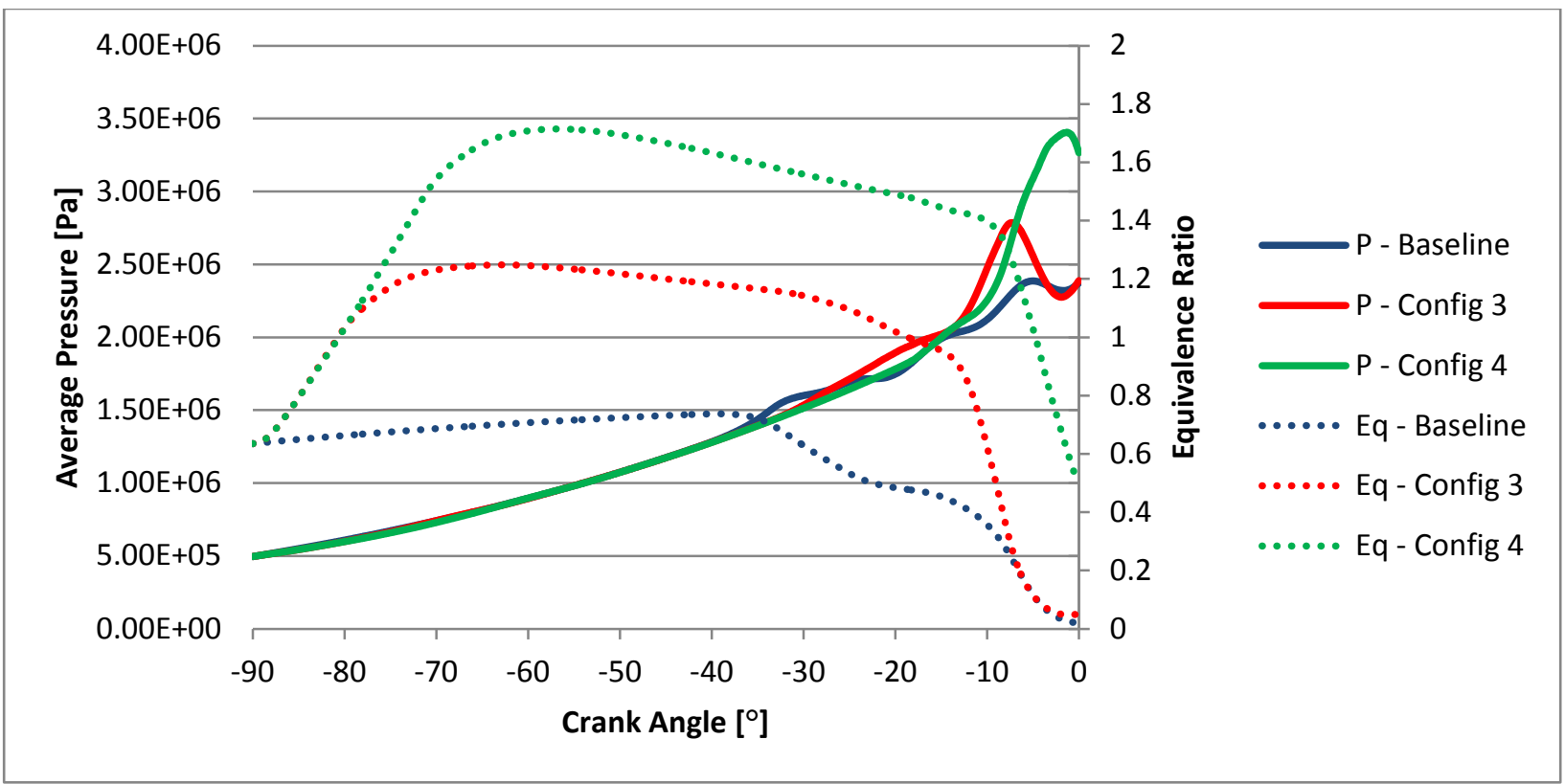

Figure C.1-Pressures and equivalence ratios for sprays beginning at $-90^{\circ} \mathrm{CA}$ with a cone angle of $30^{\circ}$ with durations of 9 and $18^{\circ} \mathrm{CA}$.

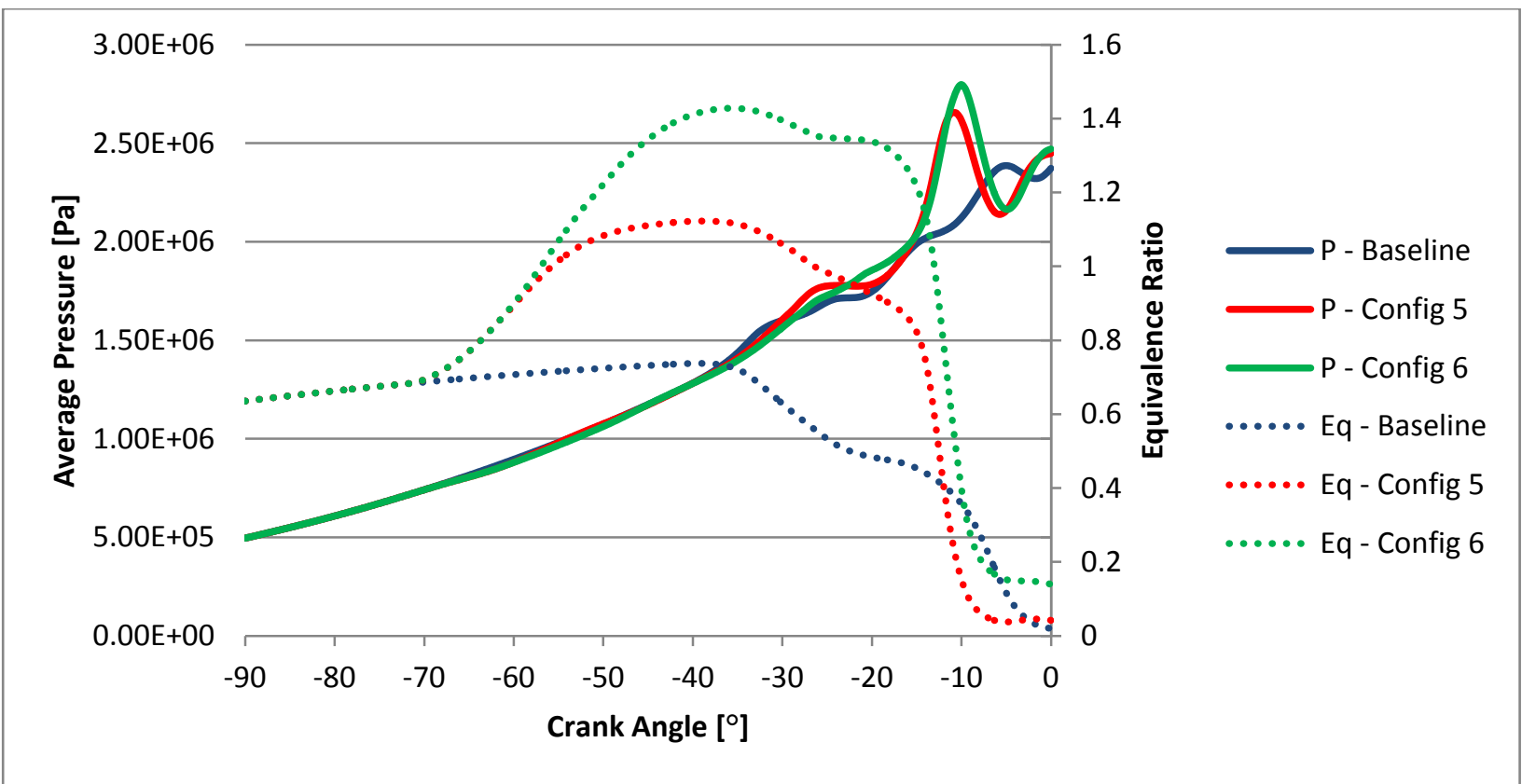

Figure C.2-Pressures and equivalence ratios for sprays beginning at $-72^{\circ} \mathrm{CA}$ with a cone angle of $10^{\circ}$ with durations of 9 and $18^{\circ} \mathrm{CA}$. 


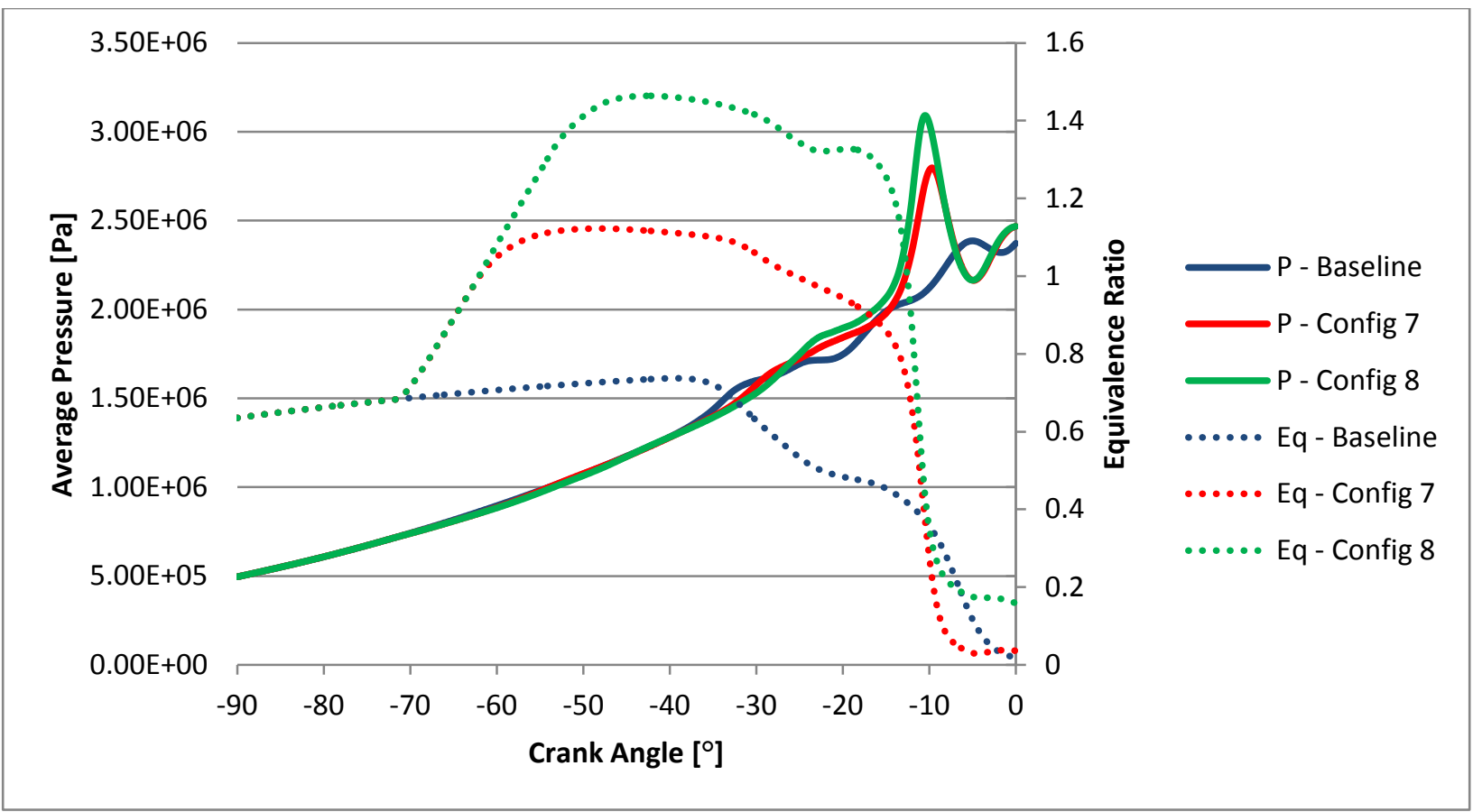

Figure C.3-Pressures and equivalence ratios for sprays beginning at $-72^{\circ} \mathrm{CA}$ with a cone angle of $30^{\circ}$ with durations of 9 and $18^{\circ} \mathrm{CA}$.

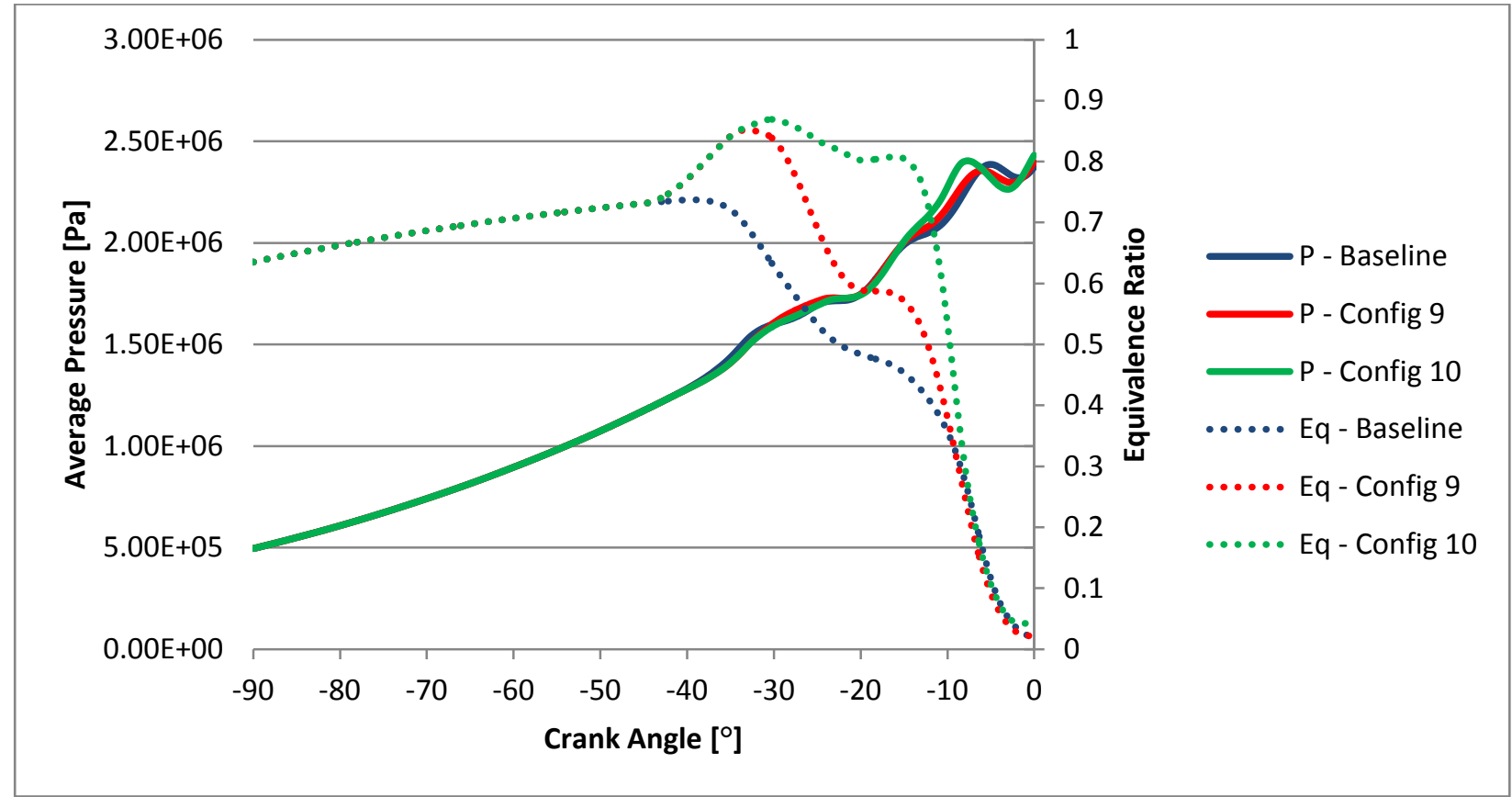

Figure C.4-Pressures and equivalence ratios for sprays beginning at $-45^{\circ} \mathrm{CA}$ with a cone angle of $10^{\circ}$ with durations of 9 and $18^{\circ} \mathrm{CA}$. 


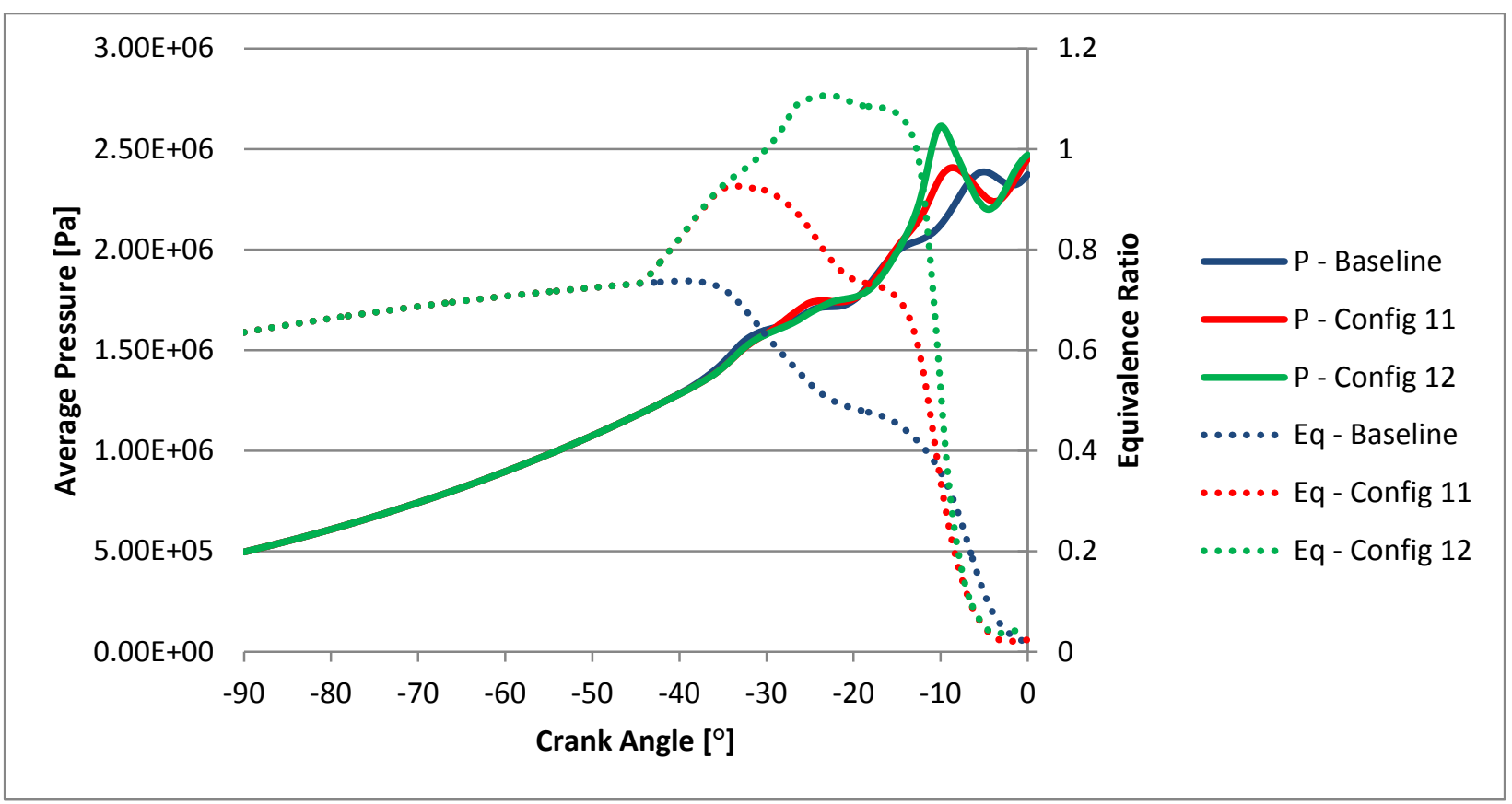

Figure C.5-Pressures and equivalence ratios for sprays beginning at $-45^{\circ} \mathrm{CA}$ with a cone angle of $30^{\circ}$ with durations of 9 and $18^{\circ} \mathrm{CA}$. 


\section{Appendix D - Spray Angle Simulations}

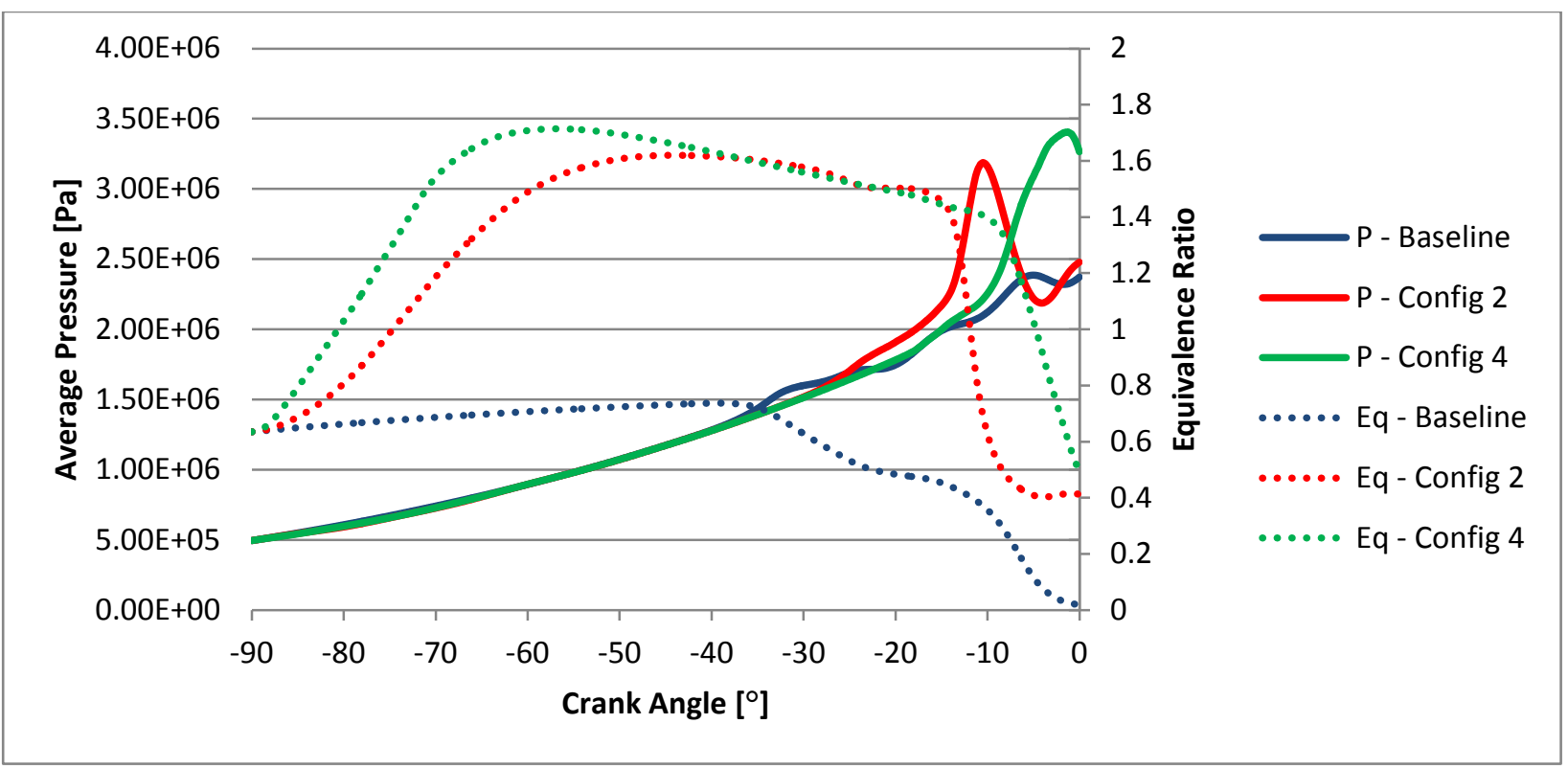

Figure D.1 - Pressures and equivalence ratios for sprays beginning at $-90^{\circ} \mathrm{CA}$ with a duration of $18^{\circ} \mathrm{CA}$ and cone angles of $10^{\circ}$ and $30^{\circ}$.

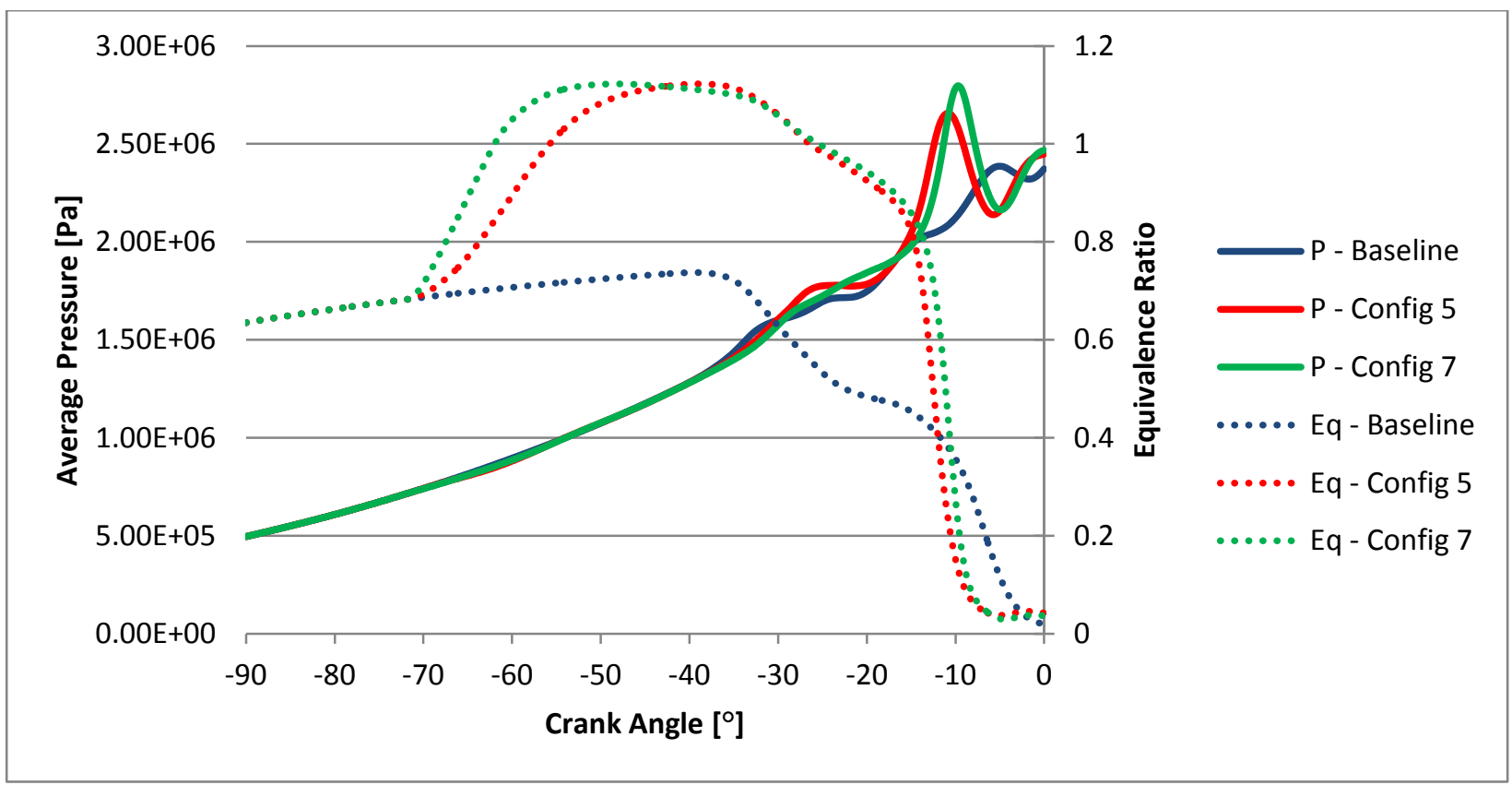

Figure D.2 - Pressures and equivalence ratios for sprays beginning at $-72^{\circ} \mathrm{CA}$ with a duration of $9^{\circ} \mathrm{CA}$ and cone angles of $10^{\circ}$ and $30^{\circ}$. 


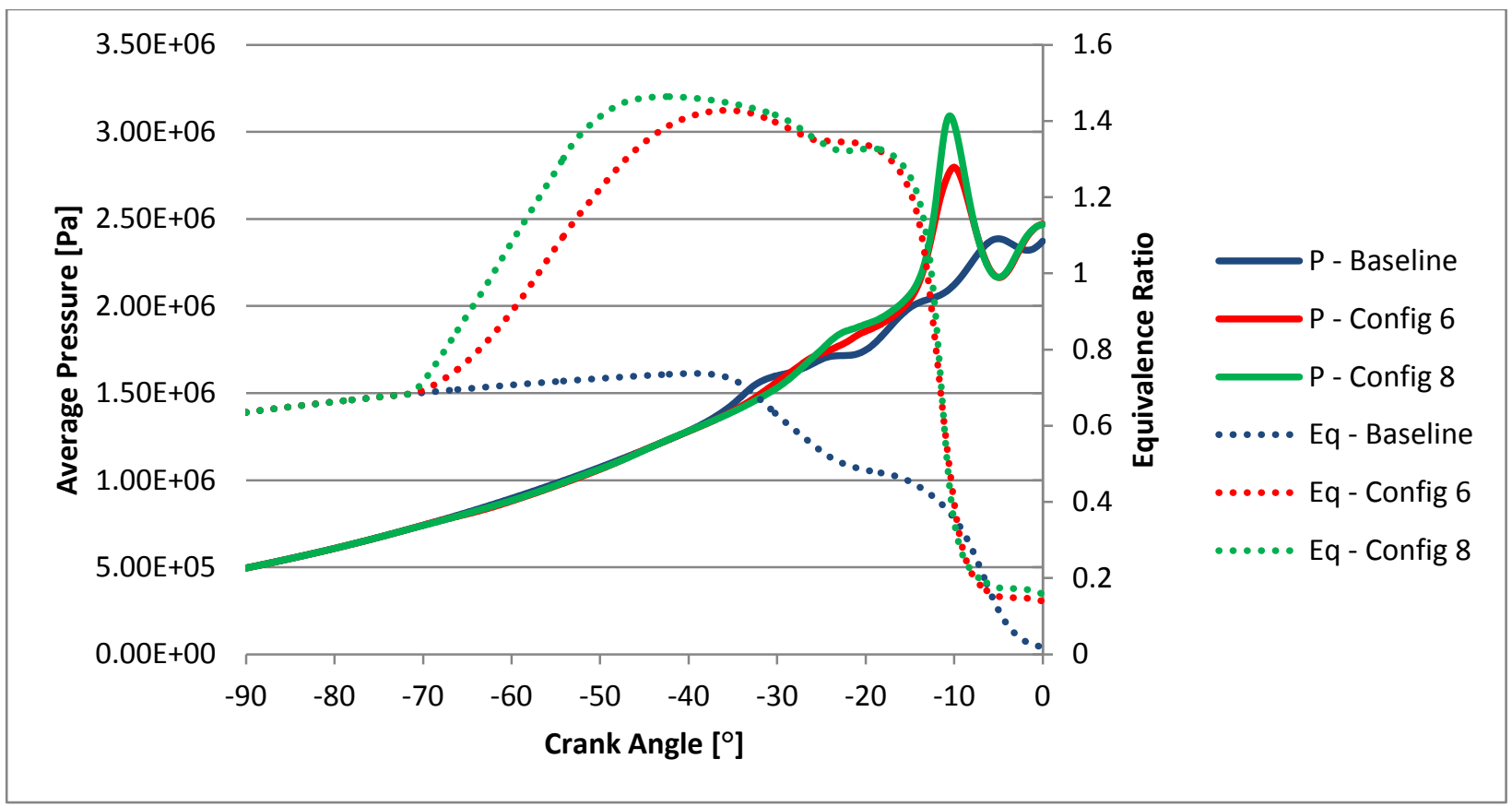

Figure D.3 - Pressures and equivalence ratios for sprays beginning at $-72^{\circ} \mathrm{CA}$ with a duration of $18^{\circ} \mathrm{CA}$ and cone angles of $10^{\circ}$ and $30^{\circ}$.

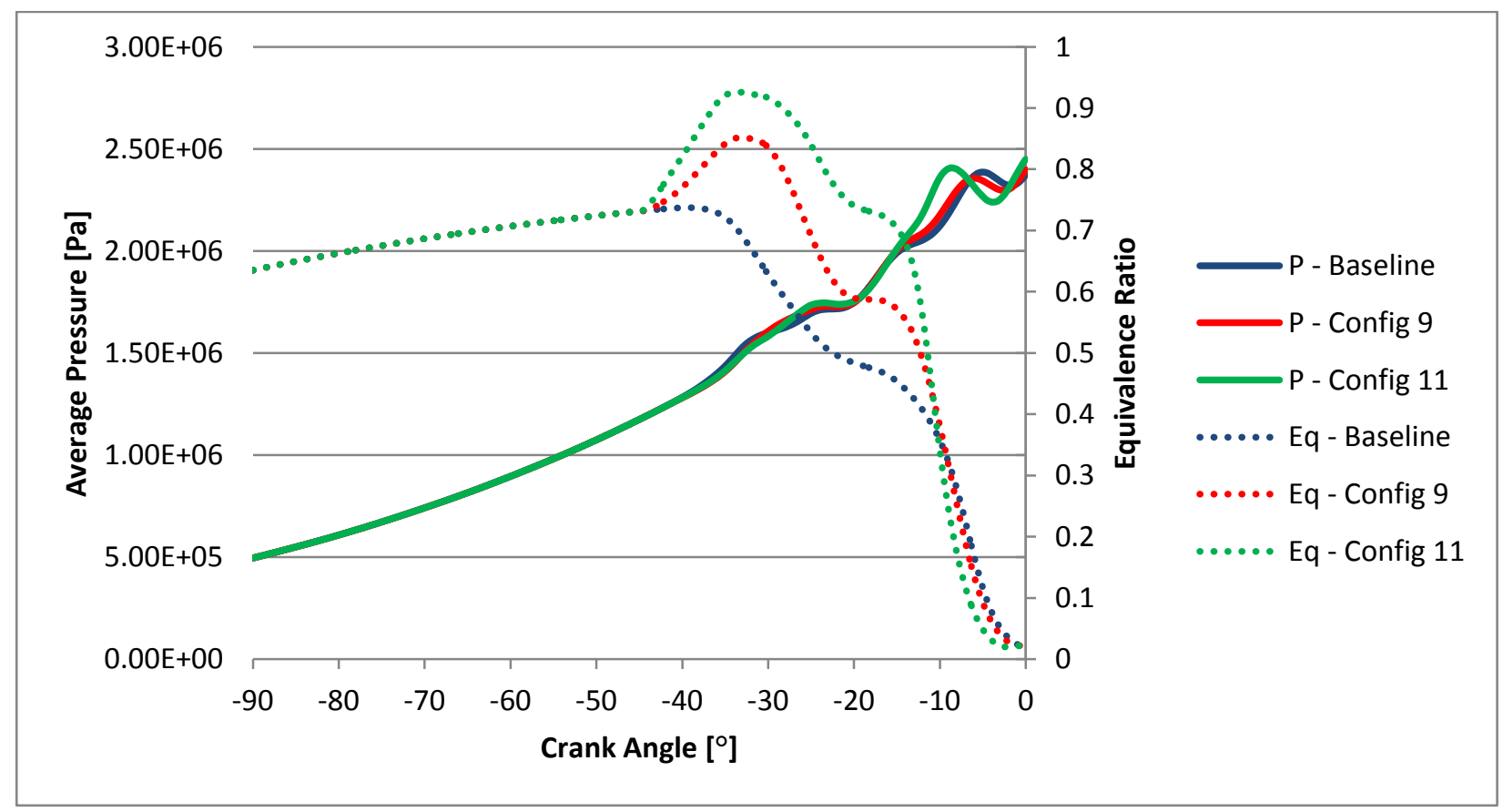

Figure D.4 - Pressures and equivalence ratios for sprays beginning at $-72^{\circ} \mathrm{CA}$ with a duration of $18{ }^{\circ} \mathrm{CA}$ and cone angles of $10^{\circ}$ and $30^{\circ}$. 


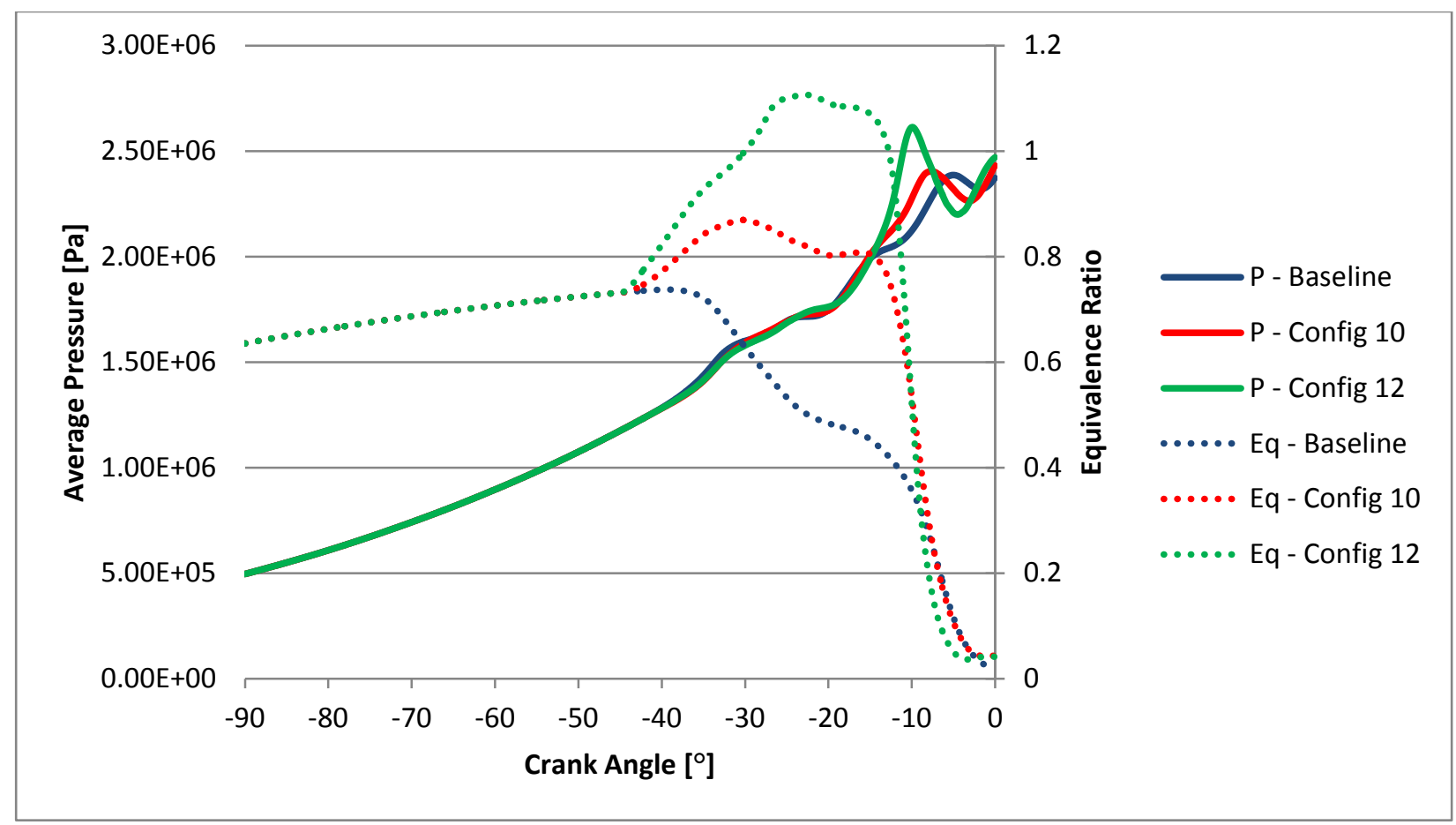

Figure D.5 - Pressures and equivalence ratios for sprays beginning at $-72^{\circ} \mathrm{CA}$ with a duration of $18^{\circ} \mathrm{CA}$ and cone angles of $10^{\circ}$ and $30^{\circ}$. 


\section{References}

[1] Shi, Y., Reitz, R. D. Chapter 15: Multi-dimensional Modelling of Diesel Combustion: Review. Lakshminarayanan, P. A., Aghav, Y. V. (eds.) Modelling Diesel Combustion, Springer Science + Business Media B.V., (2010).

[2] Celik, I., Yavuz, I., Smirnov, A. Large eddy simulations of in-cylinder turbulence I internal combustion engines: a review, International Journal of Engine Research 2, 2001, 119148.

[3] Abani, N., Kokjohn, S., Park, S., Bergin, M., Munnannur, A., Ning, W., Sun, Y., Reitz, R. D. An Improved Spray Model for Reducing Numerical Parameter Dependencies in Diesel Engine CFD Simulations, SAE Paper 2009-01-0970, 2008.

[4] Munnannur, A. Droplet collision modeling in multi-dimensional engine spray computations. Ph.D. Dissertation, University of Wisconsin-Madison, 2007.

[5] Beard, P., Duclos, J. M., Habchi, C., Bruneaux, G., Mokkadem, K., Baritaud, T. Extension of Lagrangian-Eulerian Spray Modeling: Application to High-Pressure Evaporating Diesel Sprays, SAE Paper 2000-01-1893, 2000.

[6] Johnson, N. L., Amsden, A. A., Naber, J. D., Reitz, R.D. Three-Dimensional Computer Modeling of Hydrogen Injection and Combustion. High Performance Computing Symposium, The Society for Computer Simulation, Phoenix, Arizona, 1995.

[7] Schmidt, D. P., Senecal, P. K. Improving the Numerical Accuracy of Spray Simulations, SAE Paper 2002-01-1113, 2002.

[8] Vujanovic, M. - Numerical Modelling of Multiphase Flow in Combustion of Liquid Fuel, Doctoral Thesis - University of Zagreb, 2011. 
[9] Pomraning, E., Rutland, C. J. A dynamic one-equation non-viscosity LES model, AIAA 44, 2002, 689-701.

[10] Jhavar, R. Using large eddy simulations to study DI-HCCI diesel engine flow structure, mixing and combustion. Ph.D. Dissertation, University of Wisconsin-Madison, 2007.

[11] $\mathrm{Hu}, \mathrm{B}$. Development of a general diesel combustion model in the context of large eddy simulation, Ph.D. Dissertation, University of Wisconsin-Madison, 2008.

[12] Fogleman, M., Lumley, J., Rempfer, D., Haworth, D. Application of the proper orthogonal decomposition to datasets of IC engine flows, Journal of Turbulence 5, 2004.

[13] Poinsot, T. Instabilities of Flows: With and Without Heat Transfer and Chemical Reaction, Chapter 7: Combustion and CFD for Combustion. CISM International Centre for Mechanical Sciences 517, Springer Vienna, 2010, 233-244.

[14] Haworth, D. C. A review of turbulent combustion modeling for multidimensional incylinder CFD, SAE Paper 2005-01-0993, 2005.

[15] Magnussen, B. F. On the Structure of Turbulence and a Generalized Eddy Dissipation Concept for Chemical Reaction in Turbulent Flow, 19 ${ }^{\text {th }}$ AIAA Meeting, 1981.

[16] Magussen, B. F. The Eddy Dissipation Concept: A Bridge Between Science and Technology, ECCOMAS Thematic Conference on Computational Combustion, 2005.

[17] Magnussen, B. F., Hjertager, B. H. On mathematical models of turbulent combustion with special emphasis on soot formation and combustion, $16^{\text {th }}$ International Symposium on Combustion, The Combustion Institute, 1976.

[18] Bray, K. N., Peters, N. Laminar Flamelets in Turbulent Flames. Libby, P. A., Williams, F. A (eds), Turbulent Reacting Flows, Academic Press, 1994, 63-114. 
[19] Puri, I. K., Seshadri, K., Smooke, M. D., Keyes, D. E. A comparison between numerical calculations and experimental measurements of the structure of a counterflow methane-air diffusion flame, Combustion Science and Technology Vol. 56, 1987, 1-22.

[20] Keyes, D. E., Smooke, M.D. Flame sheet starting estimates for counterflow diffusion flame problems, Journal of Computational Physics Vol. 73, No. 2, 1987, 267-288.

[21] ANSYS, Inc, ANSYS FLUENT 13.0 Theory Guide, ANSYS, Inc, 2010.

[22] Barths, H., Pitsch, H., Peters, N. 3D Simulation of DI diesel combustion and pollutant formation using a two-component reference fuel Oil \& Gas Science and Technology Vol. 54, No. 2, 1999, 233-244.

[23] Pitsch, H., Wan, Y. P., Peters, N. Numerical investigation of soot formation and oxidation under diesel engine conditions, SAE Paper 952357, 1995.

[24] Pitsch, H., Barths, H., Peters, N. Three-dimensional modeling of NOx and soot formation in DI-diesel engines using detailed chemistry based on the interactive flamelet approach, SAE Paper 962057, 1996.

[25] Barths, H., Antoni, C., Peters, N. Three-dimensional simulation of pollutant formation in a DI-diesel engine using multiple interactive flamelets, SAE Paper 982459, 1998.

[26] Keum, S., Im, H. G., Assanis, D. N. A spray-interactive flamelet model for direct injection engine combustion, Combustion Science and Technology Vol. 184, 2012, 469488.

[27] Correa, C. Combustion Simulations in Diesel Engines using Reduced Reaction Mechanisms. Combined Faculties for the Natural Sciences and for Mathematics of the Rupertus Carola University of Heidelberg, Germany, 2000. 
[28] Ravindra, H. A., Warnatz, J. Applying ILDM technique with time scaling for CFD simulation of diesel engine. Wesseling, P., Oñate E., Périaux, P. (Eds). European Conference on Computational Fluid Dynamics, 2006, 1-17.

[29] Maas, U., Pope, S. B. Implementation of simplified chemical kinetics based on intrinsic low-dimensional manifolds, Twenty-Fourth Symposium (International) on Combustion, 1992, 103-112.

[30] Maas, U., Pope, S. B. Laminar flame calculations using simplified chemical kinetics based on intrinsic low-dimensional manifolds, Twenty-Fifth Symposium (International) on Combustion, 1994, 1349-1356.

[31] J.D. Dale, M.D. Checkel, P.R. Smy, Application of High Energy Ignition Systems to Engines, Progress in Energy and Combustion Science 23, 1997, 379-398.

[32] M.C. Turkish, 3-Valve Stratified Charge Engines: Evolvement, Analysis and Progression, SAE Technical Paper 741163, 1974.

[33] T. Date, S. Yagi, Research and Development of Honda CVCC Engine, SAE Technical Paper 740605, 1974.

[34] N. Noguchi, S, Sanda, N. Nakamura, Development of Toyota Lean Burn Engine, SAE Technical Paper 760757, 1976.

[35] T.G. Adams, Torch Ignition for Combustion Control of Lean Mixtures, SAE Technical Paper 790440, 1979.

[36] W. Brandstetter, The Volkswagen Lean Burn Pc-Engine Concept, SAE Technical Paper 800456, 1980.

[37] F.A. Wyczalek, J.L. Harned, S. Maksymiuk, J.R. Blevins, EFI Prechamber Torch Ignition of Lean Mixtures, SAE Technical Paper 750351, 1975. 
[38] T.K. Garrett, Porsche stratified charge engine, Environmental Science and Technology 9 (9), 1975, 826-830.

[39] E. Toulson, H.J. Schock, W.P. Attard, A review of Pre-Chamber Initiated Jet Ignition Combustion Systems, SAE Technical Paper 2010-01-2263, 2010.

[40] J.F. Regueiro, The Case for New Divided-Chamber Diesel Combustion Systems Part One: Critical Analysis of Current DI and Past Significant Divided-Chamber Engines, SAE Technical Paper 2001-01-0271, 2001.

[41] J.F. Regueiro, The Case for New Divided-Chamber Diesel Combustion Systems Part Two: Critical Analysis of, and Solutions for, Swirl-Prechamber Engines, SAE Technical Paper 2001-01-0274, 2001.

[42] J.F. Regueiro, The Case for New Divided-Chamber Diesel Combustion Systems Part Three: Problems with Current DI Engines and Divided-Chamber Solutions for Future Diesel Engines, SAE Technical Paper 2001-01-0278, 2001.

[43] A. Jamrozik, W. Tutak, A. Kociszewski, M. Sosnowski, Numerical simulation of twostage combustion in SI engine with prechamber, Applied Mathematical Modelling 37, 2013, 2961-2982.

[44] S. Heyne, G. Millot, D. Favrat, Numerical Simulations of a Prechamber Autoignition Engine Operating on Natural Gas, International Journal of Thermodynamics Vol. 14 (No. 2), 2011, 43-50.

[45] A.A. Boni, M. Chapman, J.L. Cook, G.P. Schneyer, Computer simulation of combustion in a stratified charge engine, Symposium (International) on Combustion Vol. 16, Issue 1, $1977,1527-1541$. 
[46] S.R. Tonse, L.D. Cloutman, The effect of prechambers on flame propagation in a natural-gas powered engine, Lawrence Livermore National Lab., CA, 1995.

[47] H. Ryu, T. Asanuma, Turbulent Flame Propagation in a Spark Ignition Engine with an Unscavenged Horizontal Prechamber, SAE Technical Paper 881603, 1988.

[48] H. Ryu, T. Asanuma, Numerical Simulation of Two-Dimensional Combustion Process in a Spark Ignition Engine with a Prechamber using k- $\varepsilon$ Turbulence Model, SAE Technical Paper 890669, 1989.

[49] A.A. Boretti, Modelling auto ignition of hydrogen in a jet ignition pre-chamber, International Journal of Hydrogen Energy 35, 2010, 3881-3890.

[50] P. Pinchon, Three Dimensional Modelling of Combustion in a Prechamber Diesel Engine, SAE Technical Paper 890666, 1989.

[51] R.P. Roethlisberger, D. Favrat, Investigation of the prechamber geometrical configuration of a natural gas spark ignition engine for cogeneration: part 1. Numerical simulation, International Journal of Thermal Science 42, 2003, 223-237.

[52] F.C. Loccisano, Investigation of Optical Prechamber Spark Plug and Dual Laser Pulses for Ignition, Department of Mechanical Engineering, Colorado State University, Fort Collins, Colorado, USA, 2011.

[53] Hiroyasu, H. Diesel Engine Combustion and its Modeling. Diagnostics and Modeling of Combustion in Reciprocating Engines, COMODIA 85, Proceedings of Symposium (1985), 53-75.

[54] Dent, J. C. Basis for the Comparison of Various Experimental Methods for Studying Spray Penetration, SAE Paper 710571, 1971. 
[55] Flügel, A., Zigan, L., Schmitz, I., Wesing, M., Leipertz, A. A Model to Predict Fuel Spray Penetration at High Temperature and Pressure, ILASS - 24th European Conference on Liquid Atomization and Spray Systems, 2011, 1-9.

[56] Bekdemir, C. Numerical Modeling of Diesel Spray Formation and Combustion. Master's Thesis - Eindhoven University of Technology, 2008.

[57] Kong, S. C., Reitz, R. D. Multidimensional Modeling of Diesel Ignition and Combustion Using a Multistep Kinetics Model. Journal of Engineering for Gas Turbines and Power Vol. 115, 1993, 781-789.

[58] Kong, S. C., Han, Z., Reitz, R. D. The Development and Application of a Diesel Ignition and Combustion Model for Multidimensional Engine Simulation, SAE Paper 950278, 1995.

[59] Sazhin, S. S., Martynov, S. B., Kristyadi, T., Crua, C., Heikal, M. R. Diesel fuel spray penetration, heating, evaporation and ignition: modeling versus experimentation. International Journal of Engineering Systems and Modelling, Vol. 1, No. 1, 2008.

[60] López, J. J., Novella, R., García, Winklinger, J. F. Investigation of the ignition and combustion processes of a dual-fuel spray under diesel-like conditions using computation fluid dynamics (CFD) modeling. Mathematical and Computer Modelling, 2011.

[61] Shuai, S., Abani, N., Yoshikawa, T., Reitz, R., Park, S. W. Simulating low temperature diesel combustion with improved spray models. International Journal of Thermal Sciences, Vol. 48, 2009, 1786-1799.

[62] Baumgarten, C. Mixture Formation in Internal Combustion Engines. Springer-Verlag Berlin Heidelberg, 2006. 
[63] Schugger, C., Meingast, U., Renz, U. Time-Resolved Velocity Measurements in the Primary Breakup Zone of a High Pressure Diesel Injection Nozzle. ILASS - Europe, 2000.

[64] Walther, J., Schaller, J, K., Wirth, R., Tropea, C. Characterization of Cavitating Flow Fields in Transparent Diesel Injection Nozzles using Fluorescent Particle Image Velocimetry (FPIV), ILASS - Europe, 2000.

[65] Meingast, U., Reichelt, L., Renz, U., Müller, D., Heine, B. Nozzle Exit Velocity Measurements at a Multi-Orifice CR-Nozzle, ILASS - Europe, 2000.

[66] Naber, J. D., Siebers, D. L. Effects of gas density and vaporization on penetration and dispersion of diesel sprays, SAE Paper 960034, 1996.

[67] Siebers, D. L. Scaling liquid-phase fuel penetration in diesel sprays based on mixinglimited vaporization, SAE Paper 1999-01-0528, 1999.

[68] Chryssakis, C. A., Assanis, D. N., Tanner, F. X. Chapter 9: Atomization Models. Ashgriz, N. (ed.) Handbook of Atomization and Sprays, Springer Science + Business Media, LLC, 2011.

[69] Griffiths, J. F., Halford-Maw, P.A., Rose, D. J. Fundamental features of hydrocarbon autoignition in a rapid compression machine, Combustion and Flame 95, 1193, 291306.

[70] K. Kumar, C. Sung, An experimental study of the autoignition characteristics of conventional jet fuel/oxidizer mixtures: Jet-A and JP-8, Combustion and Flame 157, 2012, 676-685.

[71] M.P. Halstead, L.J. Kirsch, A. Prothero, C.P. Quinn, A mathematical model for hydrocarbon autoignition at high pressures, Proceedings of the Royal Society A 346, 1975, 515-538. 
[72] M.P. Halstead, L.J. Kirsch, C.P. Quinn, The Autoignition of Hydrocarbon Fuels at High Temperatures and Pressures - Fitting of a Mathematical Model, Combustion and Flame $30,1977,45-60$.

[73] R.D. Reitz, C.J. Rutland, Development and Testing of Diesel Engine CFD Models, Progress in Energy and Combustion Science 21, 1995, 173-195.

[74] S. Kong, Z. Han, R. Reitz, The Development and Application of a Diesel Ignition and Combustion Model for Multidimensional Engine Simulation, SAE Technical Paper 950278, 1995.

[75] S.S. Sazhin, S.B. Martynov, T. Kristyadi, C. Crua, M.R. Heikal, Diesel fuel spray penetration, heating, evaporation and ignition: modelling versus experimentation, International Journal of Engineering Systems Modelling and Simulation Vol. 1, No. 1, 2008.

[76] M. Ribaucour, R. Minetti, E.M. Sazhina, S.S. Sazhin, Autoigition of n-pentane in a rapid compression machine: experiment versus modelling, Third European Combustion Meeting ECM, 2007.

[77] M.A. Theobald, A Numerical Simulation of Diesel Autoignition, Department of Mechanical Engineering, Massachusetts Institute of Technology, Cambridge, Massachusetts, USA, 1986.

[78] I. Dhuchakallaya, A.P. Watkins, Self-ignition of diesel spray combustion, Heat Mass Transfer 45, 2009, 1627-1635.

[79] B. Natarajan, F.V. Bracco, On Multidimensional Modeling of Auto-Ignition in SparkIgnition Engines, Combustion and Flame 57, 1984, 179-197. 
[80] H. Schäpertöns, W. Lee, Multidimensional Modelling of Knocking Combustion in SI Engines, SAE Technical Paper 850502, 1985.

[81] T. Minagawa, H. Kosaka, T. Kamimoto, A Study of Ignition Delay of Diesel Fuel Spray via Numerical Simulation, SAE Technical Paper 2000-01-1892, 2000.

[82] K, Yoshizawa, A. Teraji, E. Aochi, M. Kubo, S. Kimura, Numerical Analysis of Combustion in Gasoline Compression Ignition Engines, SAE Technical Paper 2002-01$2865,2002$.

[83] V. Hamosfakidis, R.D. Reitz, Optimization of a hydrocarbon fuel ignition model for two single component surrogates of diesel fuel, Combustion and Flame 132, 2003, 433450.

[84] C. Baumgarten, Mixture Formation in Internal Combustion Engines, Springer-Verlag, Berlin Heidelberg, 2006.

[85] C.A. Chryssakis, C.A. Assanis, D.N. Tanner, in: N. Ashgriz (Ed.), Handbook of Atomization and Sprays, Springer Science + Business Media, LLC, 2011.

[86] Sandia National Laboratories, http://www.sandia.gov/ecn/cvdata/dsearch/frameset.php?nam=1, 2012.

[87] G. Mittal, C. Sung, Aerodynamics inside a rapid compression machine, Combustion and Flame 145, 2006, 160-180.

[88] Lee, D., Hochgreb, S. Rapid Compression Machines: Heat Transfer and Suppression of Corner Vortex, Combustion and Flame 114, 1998, 531-545.

[89] Clarkson, J., Griffiths, J. F., MacNamara, J. P., Whitaker, B. J. Temperature Fields During the Development of Combustion in a Rapid Compression Machine, Combustion and Flame 125, 2001, 1162-1175. 
[90] Griffiths, J. F., MacNamara, J. P., Sheppard, C. G. W., Turton, D. A., Whitaker, B. J. The relationship of knock during controlled autoignition to temperature inhomogeneities and fuel reactivity, Fuel 81, 2002, 2219-2225.

[91] Mittal, G., Sung, C. A Rapid Compression Machine for Chemical Kinetics Studies at Elevated Pressure and Temperatures, Combustion Science and Technology 179, 2007, 497-530.

[92] G. Mittal, M.P. Raju, C. Sung, CFD modeling of two-stage ignition in a rapid compression machine: Assessment of zero-dimensional approach, Combustion and Flame 157, 2010, 1316-1324.

[93] Roubaud, A., Favrat, D. Improving performances of a lean burn cogeneration biogas engine equipped with combustion prechambers, Fuel 84, 2005, 2001-2007.

[94] Heyne, S., Meier, M., Imbert, B., Favrat, D. Experimental investigation of prechamber autoignition in a natural gas engine for cogeneration, Fuel 88, 2009, 547-552.

[95] Roethlisberger, R. P., Favrat, D. Comparison between direct and indirect (prechamber) spark ignition in the case of a cogeneration natural gas engine, Part 1: engine geometrical parameters, Applied Thermal Engineering 22, 2002, 1217-1229.

[96] Roethlisberger, R. P., Favrat, D. Investigation of the prechamber geometrical configuration of a natural gas spark ignition engine for cogeneration: Part 2, Experimentation, Internation Journal of Thermal Science 42, 2003, 239-253. 